Classificação simplética de germes de curvas parametrizadas e estrelas lagrangianas 

SERVIÇO DE PÓS-GRADUAÇÃO DO ICMC-USP

Data de Depósito: 22/05/2015

Assinatura:

\title{
Classificação simplética de germes de curvas parametrizadas e estrelas lagrangianas
}

\author{
Fausto Assunção de Brito Lira \\ Orientadora: Profa. Dra. Roberta Godoi Wik Atique \\ Coorientador: Prof. Dr. Wojciech Domitrz
}

Tese apresentada ao Instituto de Ciências Matemáticas e de Computação - ICMC-USP, como parte dos requisitos para obtenção do título de Doutor em Ciências - Matemática. VERSÃO REVISADA

Maio de 2015 
Ficha catalográfica elaborada pela Biblioteca Prof. Achille Bassi e Seção Técnica de Informática, ICMC/USP, com os dados fornecidos pelo(a) autor(a)

Assunção de Brito Lira, Fausto
Classificaça simplética de germes de curvas
parametrizadas e estrelas lagrangianas / Fausto
Assunção de Brito Lira; orientadora Roberta Godoi
Wik Atique; co-orientador Wojciech Domitrz. -- São
Carlos, 2015.
$82 \mathrm{p.}$
Tese (Doutorado - Programa de Pós-Graduação em
Matemática) -- Instituto de Ciências Matemáticas e
de Computação, Universidade de São Paulo, 2015.
1. classificação simplética. 2. método das
restriçoses algébricas. 3. parte de quase grau mínimo
proporcional. 4. estrelas lagrangianas. I. Godoi
Wik Atique, Roberta, orient. II. Domitrz, Wojciech,
co-orient. III. Título.


Aos meus pais Carlos $e$ Anita. 



\section{Agradecimentos}

Primeiramente, agradeço a Deus pela vida, saúde, família e todas as pessoas maravilhosas que colocou em minha vida.

Agradeço aos meus pais pela criação, amor e educação.

Sou grato a minha esposa Karen que sempre esteve ao meu lado em todos os momentos do doutorado. Também por toda dedicação, além do amor e carinho.

Agradeço a minha orientadora, a professora Roberta Godoi Wik Atique, por aceitar trabalhar comigo, por ser atenciosa, paciente, por ser prestativa com minhas dúvidas matemáticas e por dedicar seu tempo a me orientar para a vida acadêmica. Tive muita sorte por trabalhar com ela, o que é para mim motivo de grande felicidade.

Dedico meus agradecimentos ao meu coorientador, o professor Wojciech Domitrz da Universidade Politécnica de Varsóvia por toda ajuda matemática, pela ótima recepção em Varsóvia, pelos momentos divertidos, pelas longas conversas sobre a vida e por me incentivar a comer comida saudável.

Não posso deixar de lembrar dos colegas e amigos que sempre estiveram presentes durante minha caminhada. Agradeço em especial aos amigos: Henry José Gullo, Antônio Andrade, José Santana, Maria Amélia e Juliana Theodoro.

Agradeço aos professores e funcionários do ICMC que sempre me atenderam com muita dedicação e vontade de ajudar.

Por fim, agradeço à Capes, CNPq e Fapesp pelo suporte financeiro. 



\section{Resumo}

Este trabalho tem como objetivo a classificação simplética de germes de curvas parametrizadas e de estrelas lagrangianas por meio do método das restrições algébricas.

Classificamos simpleticamente germes de curvas parametrizadas com semigrupos $(4,5,6)$, $(4,5,7)$ e $(4,5,6,7)$.

Introduzimos um invariante para distinguir restrições algébricas a germes de curvas parametrizadas quase homogêneas: a parte de quase grau mínimo proporcional. Através do método das restrições algébricas, este invariante é capaz de distinguir diferentes órbitas de germes de curvas parametrizadas quase homogêneas sob a ação dos germes de simplectomorfismos.

Classificamos estrelas lagrangianas duas a duas transversais com respeito ao grupo dos simplectomorfismos. 



\section{Abstract}

This work aims the symplectic classification of parametrized curve-germs and Lagrangian stars using the method of algebraic restrictions.

We classify simplecticaly parametrized curve-germs with semigroups $(4,5,6)$, $(4,5,7)$ e $(4,5,6,7)$

We introduce an invariant for algebraic restrictions to quasi-homogeneous parametrized curve-germs: the proportional minimum quasi degree part. By the method of algebraic restrictions, this invariant is able to distinguish different orbits of parameterized quasi-homogeneous curve-germs under the action of symplectomorphisms.

We classify Lagrangian stars two to two transversal with respect to the group of simplectomorphisms. 



\section{Sumário}

Introdução 1

1 O método das restrições algébricas $\quad 5$

1.1 Conceitos básicos de geometria simplética . . . . . . . . . . . . . . 5

1.2 O Método das Restrições Algébricas (Caso geral) . . . . . . . . . . . . . . . . . 9

1.3 O Método das Restrições Algébricas aplicado à curvas parametrizadas . . . . . . 14

1.4 Invariantes . . . . . . . . . . . . . . . . . . . . . . 17

2 Classificação simplética de curvas parametrizadas 21

2.1 Classificação simplética de curvas com semigrupo $(4,5,6,7) \ldots \ldots \ldots \ldots$

2.2 Classificação simplética de curvas com semigrupo $(4,5,6) \ldots \ldots \ldots \ldots$

2.3 Classificação simplética de curvas com semigrupo $(4,5,7) \ldots \ldots \ldots \ldots$. . . . 42

3 Estrelas Lagrangianas $\quad 47$

3.1 Introdução . . . . . . . . . . . . . . . . . . . . . . . . 47

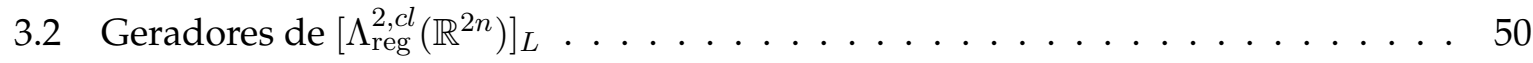

3.3 Redução ao caso linear . . . . . . . . . . . . . . . . . . . . . . . . 58

3.4 Classificação de estrelas lagrangianas em $\left(\mathbb{R}^{2 n}, 0\right) \ldots \ldots \ldots \ldots$

3.5 Índice de Maslov . . . . . . . . . . . . . . . . . . . . . . 72

4 Alguns algoritmos desenvolvidos no Singular $\quad 75$

$\begin{array}{ll}\text { Referências Bibliográficas } & 81\end{array}$ 


\section{Introdução}

Classificação é um problema central em teoria de singularidades. Consideramos a clássica relação de equivalência: germes de subconjuntos $N_{1}, N_{2} \subset\left(\mathbb{R}^{2 n}, 0\right)$ são difeomorfos se existe um germe de difeomorfismo $\Phi:\left(\mathbb{R}^{2 n}, 0\right) \rightarrow\left(\mathbb{R}^{2 n}, 0\right)$ tal que $\Phi\left(N_{1}\right)=N_{2}$. Quando os germes de subconjuntos são imagens de curvas parametrizadas, a equivalência se resume à seguinte: germes de curvas parametrizadas $f_{1}:(\mathbb{R}, 0) \rightarrow\left(\mathbb{R}^{2 n}, 0\right)$ e $f_{2}:(\mathbb{R}, 0) \rightarrow\left(\mathbb{R}^{2 n}, 0\right)$ são $\mathcal{A}$-equivalentes se existem germes de difeomorfismos $\Psi:\left(\mathbb{R}^{2 n}, 0\right) \rightarrow\left(\mathbb{R}^{2 n}, 0\right)$ e $\psi:(\mathbb{R}, 0) \rightarrow(\mathbb{R}, 0)$ tais que $\Psi \circ f_{1}=f_{2} \circ \psi$.

Agora suponha que o espaço euclidiano esteja equipado com uma estrutura adicional, nesse caso, uma estrutura simplética. Uma estrutura simplética num espaço euclidiano $\mathbb{R}^{2 n}$ é uma 2-forma fechada e não degenerada, chamada forma simplética, e um espaço simplético euclidiano é um par $\left(\mathbb{R}^{2 n}, \omega\right)$, onde $\omega$ é uma forma simplética. Neste contexto, os difeomorfismos a se considerar são os que preservam a estrutura simplética, chamados simplectomorfismos.

Fixado um espaço simplético $\left(\mathbb{R}^{2 n}, \omega\right)$ podemos definir naturalmente a seguinte relação de equivalência: germes de subconjuntos $N_{1}, N_{2} \subset\left(\left(\mathbb{R}^{2 n}, \omega\right), 0\right)$ são simplectomorfos se existe um germe de simplectomorfismo $\Phi:\left(\left(\mathbb{R}^{2 n}, \omega\right), 0\right) \rightarrow\left(\left(\mathbb{R}^{2 n}, \omega\right), 0\right)$, isto é, um germe de difeomorfismo que satisfaz $\Phi^{*} \omega=\omega$, tal que $\Phi\left(N_{1}\right)=N_{2}$. Analogamente, germes de curvas parametrizadas $f_{1}:(\mathbb{R}, 0) \rightarrow\left(\mathbb{R}^{2 n}, 0\right)$ e $f_{2}:(\mathbb{R}, 0) \rightarrow\left(\mathbb{R}^{2 n}, 0\right)$ são simplectomorfos se existem um germe de simplectomorfismo $\Psi:\left(\left(\mathbb{R}^{2 n}, \omega\right), 0\right) \rightarrow\left(\left(\mathbb{R}^{2 n}, \omega\right), 0\right)$ e um germe de difeomorfismo $\psi:(\mathbb{R}, 0) \rightarrow(\mathbb{R}, 0)$ tais que $\Psi \circ f_{1}=f_{2} \circ \psi$.

O problema de classificação simplética foi inicialmente estudado por V. I. Arnold em [A1]. Ele mostrou que a $\mathcal{A}$-órbita da singularidade $A_{2 k}$ (germe de curva parametrizada da singularidade $A_{2 k}$ ) se decompõe em $2 k+1$ órbitas simpléticas. Ele distinguiu as diferentes órbitas pela ordem de tangência lagrangiana, isto é, pela ordem de tangência da curva com a subvariedade lagrangiana mais próxima.

Em [IJ] G. Ishikawa e S. Janeczko obtiveram a classificação simplética das singularidades e curvas parametrizadas simples dadas por J. W. Bruce and T. Gaffney em [BG].

Uma singularidade simplética é simplesmente estável se é simples e permanece simples se o espaço ambiente simplético é mergulhado simpleticamente num espaço simplético de maior dimensão. Em [K] P. A. Kolgushkin obteve a classificação de singularidades simpléticas sim- 
plesmente estável de germes de curvas parametrizadas na categoria $\mathbb{C}$-analítica. Todas as singularidades simpléticas simplesmente estáveis são quase homogêneas.

Em [DJZ2] W. Domitrz, S. Janeczko e M. Zhitomirskii introduziram o método das restrições algébricas que constitui uma ferramenta para a classificação simplética. O método reduz a classificação de germes de subconjuntos quase homogêneas sob a ação dos simplectomorfismos à classificação de restrições algébricas sob ação das simetrias locais. Em particular, eles obtiveram a classificação simplética das singularidades clássicas $A-D-E$. Invariantes simpléticos de subconjuntos quase homogêneos foram descritos em termos da restrição algébrica da forma simplética do espaço simplético ambiente.

W. Domitrz adaptou em [D1] o método para o caso das restrições algébricas a germes de curvas parametrizadas quase homogêneos e obteve a classificação simplética de germes de curvas com semigrupos $(3,4,5),(3,5,7)$ e $(3,7,8)$ encontradas em [A2]. Ele obteve importantes resultados, dentre eles, que o espaço vetorial das restrições algébricas a germes de curvas parametrizadas quase homogêneas tem dimensão finita.

Em [DT1, DT2] W. Domitrz e Ż. Trębska obtiveram a classificação simplética das singularidades $T_{7}, T_{8}$ e $S_{\mu}$. A classificação simplética das singularidades $W_{8}, W_{9}$ foi obtida por $\dot{Z}$. Trębska em [T]. Todas as singularidades foram extraídas da lista de singularidades isoladas de interseções completas obtida por M. Giusti em [G2]. Em todos estes resultados foi utilizado o método das restrições algébricas.

Uma $k$-estrela lagrangiana em $\left(\left(\mathbb{R}^{2 n}, \omega\right), 0\right)$ é uma união de $k$ germes de subvariedades lagrangianas em $\left(\left(\mathbb{R}^{2 n}, \omega\right), 0\right)$ se intersectando na origem. O problema de classificação de $k$ estrelas lagrangianas sob a ação dos simplectomorfismos foi introduzido por S. Janeczko em [J]. Neste artigo ele obteve um sistema completo de invariantes para 3-estrelas lagrangianas.

Este trabalho tem como objetivo a classificação simplética de germes de curvas parametrizadas e de 3-estrelas lagrangianas por meio das restrições algébricas.

Classificamos simpleticamente germes de curvas parametrizadas com semigrupos $(4,5,6)$, $(4,5,7)$ e $(4,5,6,7)$ encontrados na lista de curvas parametrizadas irredutíveis simples de Jan Stevens em [S].

Além da ordem de tangência lagrangiana, existem 2 invariantes simpléticos: a multiplicidade simplética e índice de isotropia. Estes invariantes foram suficientes para distinguir as diferentes órbitas nas classificações acima. Entretanto, no nosso caso este sistema de invariantes não foi suficiente para distinguir as órbitas simpléticas das singularidades $(4,5,6)$ e $(4,5,6,7)$. Para solucionar este problema, introduzimos um invariante para distinguir restrições algébricas a germes de curvas parametrizadas quase homogêneas: a parte de quase grau mínimo proporcional. Conciliado ao método das restrições algébricas, este invariante é capaz de distinguir diferentes órbitas simpléticas das singularidades $(4,5,6)$ e $(4,5,6,7)$.

Conjectura: A parte de quase grau mínimo proporcional completa o sistema de invariantes acima para a classificação simplética de germes de curvas parametrizadas quase homogêneas através do método das restrições algébricas. 
Apresentamos um algoritmo desenvolvido no software Singular [DGPS] para a determinação de uma base para espaço vetorial das restrições algébricas a um germe de curva parametrizada quase homogênea. Este é um importante passo para o desenvolvimento de algoritmos que auxiliam na aplicação do método, tendo em vista que para alguns germes de curvas a classificação pode ser muito complicada. Como aplicação, utilizamos o algoritmo na classificação simplética de germes de curvas parametrizadas quase homogêneos com semigrupos $(4,5,6)$ e $(4,5,7)$.

No que concerne às estrelas lagrangianas, provamos que a classificação se reduz à classificação de matrizes ortogonais sob a ação dos isomorfismos lineares. Distinguimos as diferentes órbitas utilizando a lei de inércia de Sylvester. 


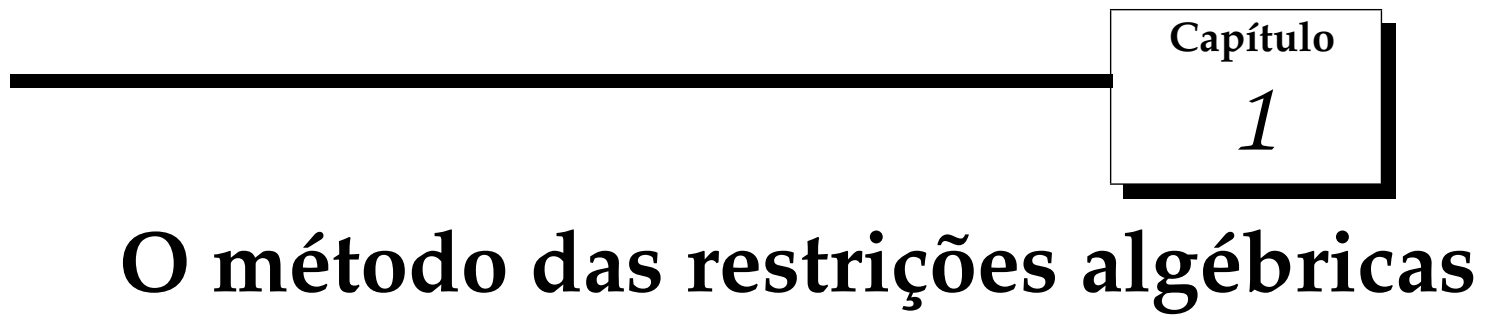

\subsection{Conceitos básicos de geometria simplética}

Nesta seção apresentamos algumas definições e resultados de geometria simplética. As definições e resultados aqui encontrados foram extraídos de [A3] e [AM1].

Sejam $V$ um espaço vetorial $m$-dimensional real e $B: V \times V \rightarrow \mathbb{R}$ uma forma bilinear . Dizemos que a forma $B$ é anti-simétrica se para todo $v, v^{\prime} \in V$ tem-se

$$
B\left(v, v^{\prime}\right)=-B\left(v^{\prime}, v\right) .
$$

O próximo resultado fornece uma forma normal para as formas bilineares anti-simétricas.

Teorema 1.1.1. ([A3]) Seja $B$ uma forma bilinear anti-simétrica em $V$. Então existe uma base $l_{1}, \ldots, l_{k}, u_{1}, \ldots, u_{n}, w_{1}, \ldots, w_{n} \in V$ tal que

$$
\begin{array}{ll}
B\left(l_{s}, v\right)=0, & \text { para todo } s=1, \ldots, k \text { e } v \in V, \\
B\left(u_{i}, u_{j}\right)=B\left(w_{i}, w_{j}\right)=0, & \text { para todo } i, j=1, \ldots, n, \\
B\left(u_{i}, w_{j}\right)=\delta_{i j}, & \text { para todo } i, j=1, \ldots, n,
\end{array}
$$

onde $\delta_{i j}$ é o delta de Kronecker.

Definição 1.1.2. Uma forma bilinear anti-simétrica $B: V \times V \rightarrow \mathbb{R}$ é dita não-degenerada, ou simplética, se

$$
B\left(v, v^{\prime}\right)=0 \text {, para todo } v \in V \Rightarrow v^{\prime}=0 \text {. }
$$


Definição 1.1.3. Um espaço vetorial simplético é um par $(V, B)$ onde $V$ é um espaço vetorial real de dimensão finita e $B: V \times V \rightarrow \mathbb{R}$ é uma forma bilinear simplética.

Observação 1.1.4. Devido ao Teorema 1.1.1 todo espaço vetorial simplético tem dimensão par.

Segue do Teorema 1.1.1 que se $B: V \times V \rightarrow \mathbb{R}$ é uma forma simplética então existe uma base de $V$ tal que

$$
B(u, w)=u^{T}\left[\begin{array}{cc}
0 & I d \\
-I d & 0
\end{array}\right] w
$$

onde $u, w \in V$.

Definição 1.1.5. Sejam $(V, B)$ um espaço vetorial simplético $2 n$ dimensional e $Y$ um subespaço de $V$. Definimos seu ortogonal simplético por

$$
Y^{B}:=\left\{v \in V \mid B\left(v, v^{\prime}\right)=0, \text { para todo } v^{\prime} \in Y\right\}
$$

Dizemos que $Y$ é

- simplético se $Y \cap Y^{B}=\{0\}$,

- isotrópico se $Y \subseteq Y^{B}$,

- coisotrópico se $Y^{B} \subseteq Y$,

- lagrangiano se $Y^{B}=Y$.

Exemplo 1.1.6. 1. Todo subespaço vetorial de $(V, B)$ de dimensão 1 é um subespaço isotrópico.

2. Um subespaço $Y$ de $(V, B)$ é coisotrópico se, e somente se, $Y^{B}$ é isotrópico. Portanto são coisotrópicos subespaços de codimensão 1 .

3. Um subespaço de $(V, B)$ é lagrangiano se ele é isotrópico, coisotrópico e sua dimensão é a metade da dimensão do espaço.

Sejam $V_{1}, V_{2}$ espaços vetoriais de dimensão finita e $\Theta$ uma forma $k$-linear em $V_{2}$. Seja $F$ : $V_{1} \rightarrow V_{2}$ uma aplicação linear. O pullback de $F$ em $\Theta$ é uma forma $k$-linear em $V_{1}$ definida por

$$
F^{*} \Theta\left(u_{1}, \ldots, u_{k}\right)=\Theta\left(F\left(u_{1}\right), \ldots, F\left(u_{k}\right)\right) .
$$

onde $u_{1}, \ldots, u_{k} \in V_{1}$.

Definição 1.1.7. Um simplectomorfismo linear entre espaços vetoriais simpléticos $(V, B)$ e $\left(V^{\prime}, B^{\prime}\right)$ é um isomorfismo $\phi: V \rightarrow V^{\prime}$ tal que $\phi^{*} B^{\prime}=B$. Neste caso dizemos que $(V, B) e\left(V^{\prime}, B^{\prime}\right)$ são linearmente simplectomorfos. 
Observação 1.1.8. A definição 1.1.7 estabelece uma relação de equivalência no conjunto de espaços vetoriais de dimensão par. De acordo com o Teorema 1.1.1, todo espaço vetorial simplético $(V, B)$ de dimensão $2 n$ é linearmente simplectomorfo ao espaço simplético $\left(\mathbb{R}^{2 n}, B_{0}\right)$, onde $B_{0}$ tem matriz associada

$$
\left[\begin{array}{cc}
0 & I d_{n} \\
-I d_{n} & 0
\end{array}\right]
$$

Seja $M$ uma variedade suave de dimensão finita. Uma $k$-forma diferencial, ou simplesmente uma $k$-forma, $\theta$ em $M$ é uma aplicação que associa a cada $p \in M$ uma forma $k$-linear alternada

$$
\theta(p): \underbrace{T_{p} M \times \cdots \times T_{p} M}_{k-\text { vezes }} \rightarrow \mathbb{R}
$$

Definição 1.1.9. Dizemos que uma $k$-forma $\theta$ em $M$ é fechada se $d \theta=0$. Dizemos que uma $k$-forma $\rho$ em $M$ é exata se existe uma $(k-1)$-forma $\tau$ tal que $\rho=d \tau$.

De acordo com o próximo resultado, toda $k$-forma fechada é localmente exata.

Teorema 1.1.10. ([AM1]) Seja M uma variedade suave de dimensão finita.

1. Toda forma exata em $M$ é fechada.

2. Se $\theta$ é uma $k$-forma fechada em $M$ então para cada $p \in M$ existe uma vizinhança $U$ de $p$ tal que $\left.\theta\right|_{U}$ é exata.

Definição 1.1.11. Uma 2-forma $\sigma$ em $M$ é simplética se $\sigma$ é fechada e a forma bilinear $\sigma(p): T_{p} M \times$ $T_{p} M \rightarrow \mathbb{R}$ é uma forma bilinear simplética, para cada $p \in M$.

Definição 1.1.12. Uma variedade simplética é um par $(M, \sigma)$ onde $M$ é uma variedade suave de dimensão finita e $\sigma$ é uma forma simplética em $M$.

Observação 1.1.13. Devido à Observação 1.1.4 toda variedade simplética tem dimensão par.

Definição 1.1.14. Sejam $(M, \sigma)$ uma variedade simplética e $N \subset M$ uma subvariedade. Dizemos que a subvariedade $N$ é:

- simplética se $T_{p} N$ é um subespaço vetorial simplético de $T_{p} M$, para todo $p \in N$;

- isotrópica se $T_{p} N$ é um subespaço vetorial isotrópico de $T_{p} M$, para todo $p \in N$;

- coisotrópica se $T_{p} N$ é um subespaço vetorial coisotrópico de $T_{p} M$, para todo $p \in N$;

- lagrangiana se $T_{p} N$ é um subespaço vetorial lagrangiano de $T_{p} M$, para todo $p \in N$. 
Exemplo 1.1.15. Sejam $n$ um inteiro positivo e $M=\mathbb{R}^{2 n}$ com coordenadas $x_{1}, \ldots, x_{n}, y_{1}, \ldots, y_{n}$. A 2-forma

$$
\omega=\sum_{i=1}^{n} d x_{i} \wedge d y_{i}
$$

é claramente simplética. Portanto $\left(\mathbb{R}^{2 n}, \omega\right)$ é uma variedade simplética.

Exemplo 1.1.16. ([AVG]) Seja L um germe de subvariedade lagrangiana. Temos que L é transversal a um hiperplano de dimensão $n$ gerado por $p_{i}$ e $q_{j}, i \in I$ e $j \in J$, onde $I, J$ são subconjuntos disjuntos de $\{1, \ldots, n\}$ tais que $I \cup J=\{1, \ldots, n\}$. Então existe um germe de função $S\left(p_{J}, q_{I}\right)$, chamada função geradora, tal que L é descrito pelas equações

$$
p_{I}=-\frac{\partial S}{\partial q_{I}} \quad \text { e } \quad q_{J}=\frac{\partial S}{\partial p_{J}}
$$

Sejam $M_{1}, M_{2}$ variedades suaves de mesma dimensão e $\theta$ uma $k$-forma em $M_{2}$. Seja $\Phi$ : $M_{1} \rightarrow M_{2}$ uma aplicação diferenciável. O pullback de $\Phi$ em $\theta$ é uma $k$-forma em $M_{1}$ definida por

$$
\Phi^{*} \theta(p)\left(u_{1}, \ldots, u_{k}\right)=\theta(\Phi(p))\left(d \Phi(p) u_{1}, \ldots, d \Phi(p) u_{k}\right)
$$

onde $p \in M_{1}$ e $u_{1}, \ldots, u_{k} \in T_{p} M_{1}$.

Definição 1.1.17. Sejam $\left(M_{1}, \sigma_{1}\right)$ e $\left(M_{2}, \sigma_{2}\right)$ variedades simpléticas de mesma dimensão. Seja $\Phi$ : $M_{1} \rightarrow M_{2}$ um difeomorfismo. Dizemos que $\Phi$ é um simplectomorfismo se $\Phi^{*} \sigma_{2}=\sigma_{1}$. Neste caso dizemos que $\left(M_{1}, \sigma_{1}\right)$ e $\left(M_{2}, \sigma_{2}\right)$ são simplectomorfos.

Analogamente ao caso linear, a Definição 1.1.17 estabelece uma relação de equivalência no conjunto das variedades simpléticas. O Teorema de Darboux estabelece uma classificação local.

Teorema 1.1.18. ([A3])(Darboux) Sejam $(M, \omega)$ uma variedade simplética e $p \in M$. Então existe uma carta local de $p\left(\mathcal{U}, x_{1}, \ldots, x_{n}, y_{1}, \ldots, y_{n}\right)$ tal que em $\mathcal{U}$

$$
\omega=\sum_{i=1}^{n} d x_{i} \wedge d y_{i} .
$$

Definição 1.1.19. Sejam $M$ uma variedade suave de dimensão finita, $\eta$ um campo de vetores de $M$ e $\theta$ uma $(k+1)$-forma em $M$, para um inteiro não negativo $k$. Então $i_{\eta} \theta$ é uma $k$-forma definida por

$$
i_{\eta} \theta(p)\left(u_{1}, \ldots, u_{k}\right)=\theta(p)\left(\eta(p), u_{1}, \ldots, u_{k}\right)
$$

onde $p \in M$ e $u_{1}, \ldots, u_{k} \in T_{p} M$. Se $f: M \rightarrow \mathbb{R}$ é uma 0 -forma então $i_{\eta} f=0$. 
Definição 1.1.20. Seja $M$ variedade suave de dimensão finita, $\theta$ uma $k$-forma em $M$ e $\eta$ um campo de vetores em $M$. A derivada de Lie de $\theta$ ao longo de $\eta$ é definida por

$$
\mathcal{L}_{\eta} \theta=i_{\eta}(d \theta)+d\left(i_{\eta} \theta\right)
$$

Proposição 1.1.21. ([A3]) Sejam $\theta_{t}$ uma família de $k$-formas, $t \in \mathbb{R}, e \Phi_{t}$ uma família de difeomorfismos tal que $\Phi_{0}=I d, t \in \mathbb{R}$. Portanto

$$
\frac{d}{d t} \Phi_{t}^{*} \theta_{t}=\Phi_{t}^{*}\left(\mathcal{L}_{\eta_{t}} \theta_{t}+\frac{d \theta_{t}}{d t}\right)
$$

onde $\eta_{t}$ satisfaz $\frac{d \Phi_{t}}{d t}=\eta_{t} \circ \Phi_{t}$

\subsection{O Método das Restrições Algébricas (Caso geral)}

Até o final deste capítulo os germes considerados são germes na categoria $C^{\infty}$ ou analítica.

Nesta seção será apresentado o método desenvolvido em [DJZ2] que é baseado numa generalização do seguinte resultado.

Teorema 1.2.1 (Darboux-Givental). ([AVG])

(i) Sejam $N$ um germe de subvariedade de $\left(\mathbb{R}^{2 n}, 0\right)$ e $\omega_{0}, \omega_{1}$ formas simpléticas em $\left(\mathbb{R}^{2 n}, 0\right)$ com mesma restrição a $T N$. Então existe um germe de difeomorfismo $\Phi:\left(\mathbb{R}^{2 n}, 0\right) \rightarrow\left(\mathbb{R}^{2 n}, 0\right)$ tal que $\Phi(x)=$ $x$ para todo $x \in N$ e $\Phi^{*} \omega_{1}=\omega_{0}$.

(ii) (Corolário de (i)) Dois germes de subvariedades de mesma dimensão $N_{1}, N_{2}$ de um espaço simplético $\left(\left(\mathbb{R}^{2 n}, \omega\right), 0\right)$ são simplectomorfos se, e somente se, $\left.\omega\right|_{T N_{2}}$ e $\left.\omega\right|_{T N_{1}}$ são difeomorfas.

Definição 1.2.2. Sejam $N_{1}$ e $N_{2}$ germes de subvariedades de $\left(\mathbb{R}^{2 n}, 0\right)$ de mesma dimensão e $\theta_{1}, \theta_{2} k$ formas em $\left(\mathbb{R}^{2 n}, 0\right)$. Dizemos que $\left.\theta_{1}\right|_{N_{1}}$ e $\left.\theta_{2}\right|_{N_{2}}$ são difeomorfas se existe um germe de difeomorfismo $\Phi:\left(\mathbb{R}^{2 n}, 0\right) \rightarrow\left(\mathbb{R}^{2 n}, 0\right)$ tal que $\Phi^{*}\left(\left.\theta_{2}\right|_{T N_{2}}\right)=\left.\theta_{1}\right|_{T N_{1}}$.

Notação: Denotamos o conjunto das formas simpléticas em $\left(\mathbb{R}^{2 n}, 0\right)$ por $\operatorname{Symp}\left(\mathbb{R}^{2 n}\right)$.

Sejam $N_{1}, N_{2}$ germes de subvariedades de $\left(\left(\mathbb{R}^{2 n}, \omega\right), 0\right)$ de dimensão $r$. Sejam $\phi_{1}:\left(\mathbb{R}^{2 n}, 0\right) \rightarrow$ $\left(\mathbb{R}^{2 n}, 0\right)$ e $\phi_{2}:\left(\mathbb{R}^{2 n}, 0\right) \rightarrow\left(\mathbb{R}^{2 n}, 0\right)$ germes de difeomorfismos tais que

$$
\phi_{1}\left(N_{1}\right)=\left(\mathbb{R}^{r} \times\{0\}, 0\right) \text { e } \phi_{2}\left(N_{2}\right)=\left(\mathbb{R}^{r} \times\{0\}, 0\right) .
$$

Denotamos, por abuso de notação, $\left(\mathbb{R}^{r}, 0\right)=\left(\mathbb{R}^{r} \times\{0\}, 0\right)$. Sejam $\sigma_{1}=\phi_{1}^{*} \omega$ e $\sigma_{2}=\phi_{2}^{*} \omega$. Como $\phi_{1}, \phi_{2}$ são germes de difeomorfismos, $\operatorname{logo} \sigma_{1}, \sigma_{2} \in \operatorname{Symp}\left(\mathbb{R}^{2 n}\right)$. Observe que $\left.\sigma_{1}\right|_{T\left(\mathbb{R}^{r}, 0\right)}$ 
e $\left.\sigma_{2}\right|_{T\left(\mathbb{R}^{r}, 0\right)}$ são difeomorfas a $\left.\omega\right|_{T N_{1}}$ e a $\left.\omega\right|_{T N_{2}}$, respectivamente. Segue do Teorema 1.2.1 (ii) que os germes $N_{1}$ e $N_{2}$ são simplectomorfos se, e somente se, $\left.\omega\right|_{T N_{1}}$ e $\left.\omega\right|_{T N_{2}}$ são difeomorfas. Por outro lado, $\left.\omega\right|_{T N_{1}}$ e $\left.\omega\right|_{T N_{2}}$ são difeomorfas se, e somente se, $\left.\sigma_{1}\right|_{T\left(\mathbb{R}^{r}, 0\right)}$ e $\left.\sigma_{2}\right|_{T\left(\mathbb{R}^{r}, 0\right)}$ são difeomorfas.

Seja Symp $\left.\left(\mathbb{R}^{2 n}\right)\right|_{\mathbb{R}^{r}}=\left\{\left.\sigma\right|_{\left(T \mathbb{R}^{r}, 0\right)}: \sigma \in \operatorname{Symp}\left(\mathbb{R}^{2 n}\right)\right\}$. O Teorema 1.2.1 (ii) reduz a classificação de germes de subvariedades de dimensão $r$ de um espaço simplético $\left(\left(\mathbb{R}^{2 n}, \omega\right), 0\right)$ com respeito ao grupo dos simplectomorfismos à classificação do conjunto $\left.\operatorname{Sym} p\left(\mathbb{R}^{2 n}\right)\right|_{\mathbb{R}^{r}}$ com respeito ao grupo dos germes de difeomorfismos $\left(\mathbb{R}^{2 n}, 0\right)$ que preservam $\left(\mathbb{R}^{r}, 0\right)$.

Definição 1.2.3. Seja $\sigma$ uma 2-forma em $\left(\mathbb{R}^{m}, 0\right)$. O posto de $\sigma$ é definido como o posto da forma bilinear $\sigma(0)$.

O seguinte resultado descreve o conjunto $\operatorname{Symp}\left(\mathbb{R}^{2 n}\right) \mid \mathbb{R}^{r}$.

Teorema 1.2.4. ([AVG]) O conjunto $\left.\operatorname{Symp}\left(\mathbb{R}^{2 n}\right)\right|_{\mathbb{R}^{r}}$ é formado pelas 2 -formas em $\left(\mathbb{R}^{r}, 0\right)$ de posto maior ou igual a $2(r-n)$.

O método das restrições algébricas foi elaborado para solucionar o seguinte problema.

Problema A. Classificar com respeito ao grupo dos simplectomorfismos as classes dos germes de variedades singulares no espaço simplético $\left(\left(\mathbb{R}^{2 n}, \omega\right), 0\right)$ que são difeomorfas a um germe de variedade singular fixa $N$.

Este problema é clássico porém de difícil trato para um germe de singularidade arbitrária. O método generaliza o Teorema de Darboux-Givental para germes de subconjuntos quase homogêneos.

Definição 1.2.5. Um germe de subconjunto $N \subset\left(\mathbb{R}^{m}, 0\right)$ é quase homogêneo se existem um sistema de coordenadas $x_{1}, \ldots, x_{m}$ de $\left(\mathbb{R}^{m}, 0\right)$ e inteiros positivos $\lambda_{1}, \ldots, \lambda_{m}$ com a seguinte propriedade: se $\left(a_{1}, \ldots, a_{m}\right) \in N$ então $\left(t^{\lambda_{1}} a_{1}, \ldots, t^{\lambda_{m}} a_{m}\right) \in N$, para todo $t \in[0,1]$. Os inteiros $\lambda_{1}, \ldots, \lambda_{m}$ são chamados pesos das variáveis $x_{1}, \ldots, x_{m}$, respectivamente.

Seja $M$ um germe de variedade suave. Denotamos por $\Lambda^{k}(M)$ o conjunto das $k$-formas em $M$. Dado um subconjunto $N \subset M$ são definidos os seguintes subespaços de $\Lambda^{k}(M)$ :

$$
\begin{gathered}
\Lambda_{N}^{k}(M)=\left\{\omega \in \Lambda^{k}(M): \omega(x)=0, \text { para todo } x \in N\right\}, \\
\mathscr{A}_{0}^{k}(N, M)=\left\{\alpha+d \beta: \alpha \in \Lambda_{N}^{k}(M), \beta \in \Lambda_{N}^{k-1}(M)\right\} .
\end{gathered}
$$

O seguinte resultado nos dá uma definição geométrica para o conjunto $\mathscr{A}_{0}^{k}(N, M)$, quando $N$ é um germe de subvariedade de $M$.

Proposição 1.2.6. ([DJZ2]) Se $N$ é um germe de subvariedade de $M$ então uma $k$-forma $\nu$ em $M$ tem restrição zero a $T N$ se, e somente se, $\nu \in \mathscr{A}_{0}^{k}(N, M)$. Portanto a restrição de uma $k$-forma $\theta \in \Lambda^{k}(M)$ a $T N$ pode ser definida como a classe de equivalência de $\theta$ no espaço $\Lambda^{k}(M)$, onde a equivalência é dada por: $\theta$ é equivalente a $\tilde{\theta}$ se $\theta-\tilde{\theta} \in \mathscr{A}_{0}^{k}(N, M)$. 
Na demonstração da Proposição 1.2.6 usa-se fortemente a estrutura suave de $N$. Portanto a definição da restrição algébrica de uma $k$-forma a um germe de subconjunto (suave ou não) é dada pela generalização da definição acima.

Definição 1.2.7. Sejam $N$ um germe de subconjunto de $M e \theta \in \Lambda^{k}(M)$. A restrição algébrica a $N$ de $\theta$ é a classe de equivalência de $\theta$ em $\Lambda^{k}(M)$, onde a equivalência é a seguinte: $\theta$ é equivalente a $\tilde{\theta}$ se, $e$ somente se, $\theta-\tilde{\theta} \in \mathscr{A}_{0}^{k}(N, M)$. A classe de equivalência de $\theta$ será denotada por $[\theta]_{N}$.

O seguinte resultado fornece um critério para a verificação se um subconjunto quase homogêneo está contido em uma subvariedade lagrangiana em $\left(\left(\mathbb{R}^{2 n}, \omega\right), 0\right)$.

Teorema 1.2.8. ([DJZ2]) Um germe de subconjunto quase homogêneo $N \subset\left(\left(\mathbb{R}^{2 n}, \omega\right), 0\right)$ está contido num germe de subvariedade lagrangiana se, e somente se, a forma simplética $\omega$ tem restrição algébrica a $N$ nula.

Observação 1.2.9. Não é difícil provar que se $\theta \in \mathscr{A}_{0}^{k}(N, M)$ então $d \theta \in \mathscr{A}_{0}^{k+1}(N, M)$. Além disso, se $\theta_{1}$ é uma $k$-forma com $\left[\theta_{1}\right]_{N}=0$ então $\left[\theta_{1} \wedge \theta_{2}\right]_{N}=0$ para toda $q$-forma $\theta_{2}$. Assim se $\theta_{1}$ é uma $k$-forma e se $\theta_{2}$ é uma q-forma então estão bem definidos: $d\left[\theta_{1}\right]_{N}:=\left[d \theta_{1}\right]_{N} e\left[\theta_{1}\right]_{N} \wedge\left[\theta_{2}\right]_{N}:=\left[\theta_{1} \wedge \theta_{2}\right]_{N}$.

Sejam $M$ e $\widetilde{M}$ germes de variedades suaves de mesma dimensão e $\Phi: \widetilde{M} \rightarrow M$ um germe de difeomorfismo. Dado $N \subset M$, é fácil verificar que $\Phi^{*} \mathscr{A}_{0}^{k}(N, M)=\mathscr{A}_{0}^{k}\left(\Phi^{-1}(N), \widetilde{M}\right)$. Portanto se $\theta$ é uma $k$-forma em $M$, o pullback de $\Phi$ a $[\theta]_{N}$ é definido por $\Phi^{*}\left([\theta]_{N}\right):=\left[\Phi^{*} \theta\right]_{\Phi^{-1}(N)}$.

Definição 1.2.10. Sejam $\theta$ e $\tilde{\theta}$-formas em $M$ e $\widetilde{M}$, respectivamente, e $N \subset M e \tilde{N} \subset \tilde{M}$ germes de subconjuntos. As restrições algébricas $[\theta]_{N} e[\widetilde{\theta}]_{\widetilde{N}}$ são difeomorfas se existe um germe de difeomorfismo $\Phi: \widetilde{M} \rightarrow M$ tal que $\Phi(\widetilde{N})=N$ e $\Phi^{*}[\theta]_{N}=[\widetilde{\theta}]_{\tilde{N}}$. Quando $M=\widetilde{M}$ e $N=\widetilde{N}$ o germe de difeomorfismo $\Phi$ é chamado uma simetria local de $N$.

Se um germe de subconjunto $N$ está contido num germe de subvariedade $M \subset\left(\mathbb{R}^{m}, 0\right)$ então o espaço vetorial $\Lambda^{k}\left(\mathbb{R}^{m}, 0\right) / \mathscr{A}_{0}^{k}\left(N,\left(\mathbb{R}^{m}, 0\right)\right)$ pode ser identificado com o espaço vetorial $\Lambda^{k}(M) / \mathscr{A}_{0}^{k}(N, M)$ devido ao seguinte resultado.

Proposição 1.2.11. ([DJZ2]) Sejam $N$ um germe de subconjunto contido num germe de subvariedade $M \subset\left(\mathbb{R}^{m}, 0\right)$ e $\theta_{1}, \theta_{2} \in \Lambda^{k}\left(\mathbb{R}^{m}, 0\right)$. Então $\left[\theta_{1}\right]_{N}=\left[\theta_{2}\right]_{N}$ se, e somente se, $\left[\left.\theta_{1}\right|_{T M}\right]_{N}=\left[\left.\theta_{2}\right|_{T M}\right]_{N}$.

A seguir vemos que as órbitas de $[\theta]_{N} \mathrm{e}\left[\left.\theta\right|_{T M}\right]_{N}$, com respeito à ação das simetrias locais de $N$, podem ser identificadas.

Proposição 1.2.12. ([DJZ2]) Sejam $N_{1}, N_{2}$ germes de subconjuntos contidos em subvariedades de mesma dimensão $M_{1}, M_{2} \subset\left(\mathbb{R}^{m}, 0\right)$, respectivamente, e $\theta_{1}, \theta_{2} \in \Lambda^{k}\left(\mathbb{R}^{m}, 0\right)$. As restrições algébricas $\left[\theta_{1}\right]_{N_{1}}$ e $\left[\theta_{2}\right]_{N_{2}}$ são difeomorfas se, e somente se, as restrições algébricas $\left[\left.\theta_{1}\right|_{T M_{1}}\right]_{N_{1}}$ e $\left[\left.\theta_{2}\right|_{T M_{2}}\right]_{N_{2}}$ são difeomorfas.

O método das restrições algébricas é baseado no seguinte Teorema: 
Teorema A (i) ([DJZ2]) Seja $N$ um germe de subconjunto quase homogêneo de $\left(\mathbb{R}^{2 n}, 0\right)$. Sejam $\omega_{0} e$ $\omega_{1}$ formas simpléticas em $\left(\mathbb{R}^{2 n}, 0\right)$ com mesma restrição algébrica a $N$. Então existe um germe de difeomorfismo $\Phi:\left(\mathbb{R}^{2 n}, 0\right) \rightarrow\left(\mathbb{R}^{2 n}, 0\right)$ tal que $\Phi(x)=x$ para todo $x \in N$ e $\Phi^{*} \omega_{1}=\omega_{0}$.

(ii) (Corolário de (i)) Dois germes de subconjuntos quase homogêneos $N_{1}, N_{2}$ num espaço simplético $\left(\left(\mathbb{R}^{2 n}, \omega\right), 0\right)$ são simplectomorfos se, e somente se, as restrições algébricas da forma simplética $\omega$ a $N_{1}$ e $N_{2}$ são difeomorfas.

O Teorema A estabelece uma correspondência entre a classificação de germes de conjuntos quase homogêneos sob a ação dos simplectomorfismos e a classificação de restrições algébricas de formas simpléticas a conjuntos quase homogêneos sob a ação das simetrias locais. No caso de subconjuntos difeomorfos a um subconjunto quase homogêneo, temos o seguinte resultado.

Corolário 1.2.13. Sejam $N_{1}, N_{2}$ germes de subconjuntos de $\left(\left(\mathbb{R}^{2 n}, \omega\right), 0\right)$ difeomorfos a um germe de subconjunto quase homogêneo $N$ de $\left(\left(\mathbb{R}^{2 n}, \omega\right), 0\right)$. Então $N_{1}$ e $N_{2}$ são simplectomorfos se, e somente se, as restrições algébricas de $\omega$ a $N_{1}$ e $N_{2}$ são difeomorfas.

Demonstração. Suponhamos que $N_{1}$ e $N_{2}$ são simplectomorfos, ou seja, existe um simplectomorfismo $\Psi:\left(\left(\mathbb{R}^{2 n}, \omega\right), 0\right) \rightarrow\left(\left(\mathbb{R}^{2 n}, \omega\right), 0\right)$ tal que $\Psi\left(N_{2}\right)=N_{1}$. Segue que

$$
\Psi^{*}[\omega]_{N_{1}}=\left[\Psi^{*} \omega\right]_{\Psi^{-1}\left(N_{1}\right)}=[\omega]_{N_{2}}
$$

Portanto $[\omega]_{N_{1}}$ e $[\omega]_{N_{2}}$ são difeomorfas.

Reciprocamente, suponhamos que $[\omega]_{N_{1}}$ e $[\omega]_{N_{2}}$ são difeomorfas. Considere os germes de difeomorfismos $\phi_{1}:\left(\mathbb{R}^{2 n}, 0\right) \rightarrow\left(\mathbb{R}^{2 n}, 0\right)$ e $\phi_{2}:\left(\mathbb{R}^{2 n}, 0\right) \rightarrow\left(\mathbb{R}^{2 n}, 0\right)$ tais que

$$
\phi_{1}\left(N_{1}\right)=N \text { e } \phi_{2}\left(N_{2}\right)=N
$$

Sejam $\omega_{1}:=\left(\phi_{1}^{-1}\right)^{*} \omega$ e $\omega_{2}:=\left(\phi_{2}^{-1}\right)^{*} \omega$. Temos que $\left[\omega_{1}\right]_{N}$ e $\left[\omega_{2}\right]_{N}$ são difeomorfas. Seja $\rho$ : $\left(\mathbb{R}^{2 n}, 0\right) \rightarrow\left(\mathbb{R}^{2 n}, 0\right)$ a simetria local de $N$ tal que

$$
\rho^{*}\left[\omega_{1}\right]_{N}=\left[\rho^{*} \omega_{1}\right]_{\rho^{-1}(N)}=\left[\omega_{2}\right]_{N} .
$$

Segue do Teorema A (i) que existe um germe de difeomorfismo $\Phi:\left(\mathbb{R}^{2 n}, 0\right) \rightarrow\left(\mathbb{R}^{2 n}, 0\right)$ tal que $\left.\Phi\right|_{N}=I d$ e $\Phi^{*} \omega_{2}=\rho^{*} \omega_{1}$. Considere o germe de difeomorfismo $T=\phi_{2}^{-1} \circ \Phi \circ \rho^{-1} \circ \phi_{1}$. Temos que

$$
\begin{aligned}
& T^{*} \omega=\left(\phi_{2}^{-1} \circ \Phi \circ \rho^{-1} \circ \phi_{1}\right)^{*} \omega=\phi_{1}^{*} \circ\left(\rho^{-1}\right)^{*} \circ \Phi^{*} \circ\left(\phi_{2}^{-1}\right)^{*} \omega=\omega \mathrm{e} \\
& T\left(N_{1}\right)=\left(\phi_{2}^{-1} \circ \Phi \circ \rho^{-1} \circ \phi_{1}\right)\left(N_{1}\right)=N_{2} .
\end{aligned}
$$


Teorema 1.2.14. ([DJZ2]) Sejam $N \subset\left(\mathbb{R}^{2 n}, 0\right)$ e $r$ a dimensão mínima de um germe de subvariedade de $\left(\mathbb{R}^{2 n}, 0\right)$ que contém $N$. Seja $M$ um germe de subvariedade em $\left(\mathbb{R}^{2 n}, 0\right)$ com dimensão $r$ com tal propriedade. A restrição algébrica $[\sigma]_{N}$ de uma 2-forma fechada $\sigma$ em $M$ tem um representante simplético em $\left(\mathbb{R}^{2 n}, 0\right)$ se, e somente se, $\operatorname{posto}(\sigma) \geq 2 r-2 n$.

Definição 1.2.15. Um germe de função, de k-forma ou de um campo de vetores $\tau$ em $\left(\mathbb{R}^{m}, 0\right)$ é quase homogêneo num sistema de coordenadas $\left(x_{1}, \ldots, x_{m}\right)$ em $\mathbb{R}^{m}$ com pesos positivos $\left(\lambda_{1}, \ldots, \lambda_{m}\right)$ se $\mathcal{L}_{E} \tau=\delta \tau$, onde $E=\sum_{i=1}^{m} \lambda_{i} x_{i} \partial / \partial x_{i}$ é o germe de campo de vetores de Euler em $\mathbb{R}^{m}$. O número real $\delta$ é chamado de quase grau de $\tau$.

É fácil mostrar que $\tau$ é quase homogêneo num sistema de coordenadas $\left(x_{1}, \ldots, x_{m}\right)$ com pesos $\left(\lambda_{1}, \ldots, \lambda_{m}\right)$ se, e somente se, $F_{t}^{*} \tau=t^{\delta} \tau$, onde $F_{t}\left(x_{1}, \ldots, x_{m}\right)=\left(t^{\lambda_{1}} x_{1}, \ldots, t^{\lambda_{m}} x_{m}\right)$. Então germes de funções quase homogêneos de quase grau $\delta$ são germes de polinômios quase homogêneos de quase grau $\delta$. A $k$-forma diferencial $\sum f_{i_{1} \cdots i_{k}} d x_{i_{1}} \wedge \cdots \wedge d x_{i_{k}}$ é quase homogênea de quase grau $\delta$ se os germes de funções $f_{i_{1} \cdots i_{k}}$ são germes de polinômios quase homogêneos de quase grau $\delta-\sum_{j=1}^{k} \lambda_{i_{j}}$. Os coeficientes $f_{i}$ de um campo de vetores quase homogêneo $\sum_{i=1}^{m} f_{i} \partial / \partial x_{i}$ de quase grau $\delta$ são germes de polinômios de quase grau $\delta+\lambda_{i}$.

Sejam $N$ um germe de subconjunto quase homogêneo em $\left(\mathbb{R}^{m}, 0\right)$ e $\theta=\sum f_{i_{1} \cdots i_{k}} d x_{i_{1}} \wedge \cdots \wedge$ $d x_{i_{k}} \in \Lambda^{k}\left(\mathbb{R}^{m}, 0\right)$. Dado $r$ um inteiro não negativo, definimos a parte quase homogênea de quase grau $r$ de $\theta$ por

$$
\theta^{(r)}:=\sum f_{i_{1} \cdots i_{k}}^{(s)} d x_{i_{1}} \wedge \cdots \wedge d x_{i_{k}} \in \Lambda^{k}\left(\mathbb{R}^{m}, 0\right),
$$

onde $s=r-i_{1}-\cdots-i_{k}$ e $f_{i_{1} \cdots i_{k}}^{(s)}$ é a parte quase homogênea de quase grau $s$ de $f_{i_{1} \cdots i_{k}}$ obtida por meio da expansão de Taylor. É fácil verificar que se a função suave $h: \mathbb{R}^{m} \rightarrow \mathbb{R}$ se anula em $N$ então $h^{(r)}$ também se anula em $N$, para todo inteiro não negativo $r$. Essa simples observação nos fornece o seguinte resultado.

Proposição 1.2.16. Se $\theta$ é uma $k$-forma em $\left(\mathbb{R}^{m}, 0\right) \operatorname{com}[\theta]_{N}=0$ então $\left[\theta^{(r)}\right]_{N}=0$.

Devido à Proposição 1.2.16 definimos a parte quase homogênea de uma restrição algébrica de uma $k$-forma.

Definição 1.2.17. Seja $a=[\theta]_{N}$ a restrição algébrica a um germe de um subconjunto quase homogêneo $N \subset\left(\mathbb{R}^{m}, 0\right)$ de uma $k$-forma $\theta$. Definimos a parte quase homogênea de quase grau $r$ de a por $a^{(r)}:=$ $\left[\theta^{(r)}\right]_{N}$. Quando $a=a^{(r)}$ dizemos que a é quase homogênea de quase grau $r$.

Proposição 1.2.18. ([D1]) Sejam $\eta$ um germe de campo de vetores quase homogêneo de quase grau i e $\theta$ um germe de uma $k$-forma de quase grau $j$ então $\mathcal{L}_{\eta} \theta$ é um germe de uma $k$-forma diferencial de quase grau $i+j$. 
Proposição 1.2.19 (Lema de Hadamard). ([G1]) Sejam U uma vizinhança convexa de 0 em $\mathbb{R}^{m}$ ef uma função suave definida em $U \times \mathbb{R}^{q}$ que se anula em $\{0\} \times \mathbb{R}^{q}$. Então existem germes de funções $f_{1}, \ldots, f_{m}$ em $U \times \mathbb{R}^{q}$ tais que

$$
f=x_{1} f_{1}+\cdots+x_{m} f_{m}
$$

onde $x_{1}, \ldots, x_{m}$ são as coordenadas do espaço $\mathbb{R}^{m}$.

\subsection{O Método das Restrições Algébricas aplicado à curvas parametri- zadas}

Os resultados aqui apresentados foram extraídos de [D1].

Domitrz em [D1] aprimorou o método das restrições algébricas quando o subconjunto quase homogêneo $N$ é a imagem de uma curva parametrizada quase homogênea no espaço simplético $\left(\left(\mathbb{R}^{2 n}, \omega\right), 0\right)$. Consideramos ao longo desta seção $\left(\mathbb{R}^{2 n}, \omega\right)$ com coordenadas $x_{1}, \ldots, x_{2 n}$ e $\omega=\sum_{i=1}^{n} d x_{i} \wedge d x_{n+i}$.

Dizemos que germes de curvas parametrizadas $f_{1}:(\mathbb{R}, 0) \rightarrow\left(\mathbb{R}^{2 n}, 0\right)$ e $f_{2}:(\mathbb{R}, 0) \rightarrow\left(\mathbb{R}^{2 n}, 0\right)$ são simplectomorfos se existem um germe de difeomorfismo $\phi:(\mathbb{R}, 0) \rightarrow(\mathbb{R}, 0)$ e um germe de simplectomorfismo $\Phi:\left(\left(\mathbb{R}^{2 n}, \omega\right), 0\right) \rightarrow\left(\left(\mathbb{R}^{2 n}, \omega\right), 0\right)$ tais que $\Phi \circ f_{1}=f_{2} \circ \phi$.

Definição 1.3.1. Um germe de curva $g:(\mathbb{R}, 0) \rightarrow\left(\mathbb{R}^{m}, 0\right)$ é quase homogêneo se existem um sistema de coordenadas tem $(\mathbb{R}, 0)$ e inteiros positivos $\lambda_{1}, \ldots, \lambda_{m}$ tais que

$$
d g\left(t \frac{d}{d t}\right)=E \circ g
$$

onde $E=\sum_{i=1}^{m} \lambda_{i} x_{i} \partial / \partial x_{i}$ é o campo de Euler em $\left(\mathbb{R}^{m}, 0\right)$. O sistema de coordenadas $\left(x_{1}, \ldots, x_{m}\right)$ é chamado quase homogêneo e os números $\lambda_{1}, \ldots, \lambda_{m}$ são chamados de pesos das variáveis $x_{1}, \ldots, x_{m}$.

Definição 1.3.2. Os inteiros positivos $\lambda_{1}, \ldots, \lambda_{m}$ são linearmente dependentes sobre inteiros não negativos se existem $j \in\{1, \ldots, m\}$ e inteiros não negativos $k_{1}, \ldots, k_{j-1}, k_{j+1}, \ldots, k_{n}$ tais que $\lambda_{j}=$ $\sum_{i \neq j} k_{i} \lambda_{i}$. Caso contrário, dizemos que $\lambda_{1}, \ldots, \lambda_{m}$ são linearmente independentes sobre inteiros não negativos.

O próximo resultado permite associar a uma curva parametrizada quase homogênea um semigrupo.

Proposição 1.3.3. Um germe de curva $g:(\mathbb{R}, 0) \rightarrow\left(\mathbb{R}^{m}, 0\right)$ é quase homogêneo se, e somente se, $g$ é $\mathcal{A}$-equivalente a

$$
t \rightarrow\left(t^{\lambda_{1}}, \ldots, t^{\lambda_{s}}, 0, \ldots, 0\right),
$$

onde $\lambda_{1}<\cdots<\lambda_{s}$ são inteiros positivos linearmente independentes sobre inteiros não negativos. Os inteiros $\lambda_{1}, \ldots, \lambda_{s}$ geram o semigrupo da curva g o qual denotaremos por $\left(\lambda_{1}, \ldots, \lambda_{s}\right)$. 
Notação: Sejam $g:(\mathbb{R}, 0) \rightarrow\left(\mathbb{R}^{m}, 0\right)$ um germe de curva parametrizada e $\theta \in \Lambda^{k}\left(\mathbb{R}^{m}, 0\right)$, $k \geq 0$. Chamaremos, por simplicidade, a restrição algébrica a $g$ de $\theta$ quando nos referimos a $[\theta]_{\operatorname{Im}(g)}$. Também por simplicidade, denotaremos $[\theta]_{\operatorname{Im}(g)}$ simplesmente por $[\theta]_{g}$.

Observação 1.3.4. Sejam $g_{1}:(\mathbb{R}, 0) \rightarrow\left(\mathbb{R}^{m}, 0\right), g_{2}:(\mathbb{R}, 0) \rightarrow\left(\mathbb{R}^{m}, 0\right)$ germes de curvas e $\phi:$ $(\mathbb{R}, 0) \rightarrow(\mathbb{R}, 0)$ um germe de difeomorfismo tais que $g_{1}=g_{2} \circ \phi$. Segue da definição de restrição algébrica que $[\theta]_{g_{1}}=[\theta]_{g_{2}}$, para toda $k$-forma $\theta$, onde $k \geq 0$.

Definição 1.3.5. Sejam $g:(\mathbb{R}, 0) \rightarrow\left(\mathbb{R}^{m}, 0\right), \tilde{g}:(\mathbb{R}, 0) \rightarrow\left(\mathbb{R}^{m}, 0\right)$ germes de curvas e $\theta, \tilde{\theta} \in$ $\Lambda^{k}\left(\mathbb{R}^{m}, 0\right)$. As restrições algébricas $[\theta]_{g} e[\tilde{\theta}]_{\tilde{g}}$ são difeomorfas se existem germes de difeomorfismos $\Phi:\left(\mathbb{R}^{m}, 0\right) \rightarrow\left(\mathbb{R}^{m}, 0\right)$ e $\phi:(\mathbb{R}, 0) \rightarrow(\mathbb{R}, 0)$ tais que $\Phi \circ g=\tilde{g} \circ \phi e \Phi^{*}[\tilde{\theta}]_{\tilde{g}}=\left[\Phi^{*} \tilde{\theta}\right]_{\Phi^{-1} \circ \tilde{g}}=$ $[\theta]_{g \circ \phi^{-1}}=[\theta]_{g}$. Quando $\operatorname{Im}(g)=\operatorname{Im}(\tilde{g})$ o germe de difeomorfismo $\Phi$ é chamado de uma simetria local de $g$.

Convenção: Até o fim desta seção consideramos $g:(\mathbb{R}, 0) \rightarrow\left(\mathbb{R}^{2 n}, 0\right)$ um germe de curva parametrizada quase homogênea.

Sejam $f_{1}, f_{2}$ germes de curvas parametrizadas $\mathcal{A}$-equivalentes a $g$.

Corolário 1.3.6. (Corolário do Teorema A) Os germes de curvas $f_{1}$ e $f_{2}$ são simplectomorfos se, $e$ somente se, $[\omega]_{f_{1}} e[\omega]_{f_{2}}$ são difeomorfas.

Demonstração. Suponhamos que existam um germe de difeomorfismo $\phi:(\mathbb{R}, 0) \rightarrow(\mathbb{R}, 0)$ e um germe de simplectomorfismo $\Phi:\left(\left(\mathbb{R}^{2 n}, \omega\right), 0\right) \rightarrow\left(\left(\mathbb{R}^{2 n}, \omega\right), 0\right)$ tais que

$$
\Phi \circ f_{1}=f_{2} \circ \phi
$$

Portanto $\Phi^{*}[\omega]_{f_{2}}=[\omega]_{\Phi^{-1} \circ f_{2}}=[\omega]_{f_{1} \circ \phi^{-1}}=[\omega]_{f_{1}}$. Logo $[\omega]_{f_{1}}$ e $[\omega]_{f_{2}}$ são difeomorfas.

Reciprocamente, suponhamos que $[\omega]_{f_{1}}$ e $[\omega]_{f_{2}}$ sejam difeomorfas. Como $f_{1}$ e $f_{2}$ são $\mathcal{A}$ equivalentes a $g$, então existem germes de difeomorfismos $\Phi_{1}, \Phi_{2}$ em $\left(\mathbb{R}^{2 n}, 0\right)$ e $\phi_{1}, \phi_{2}$ em $(\mathbb{R}, 0)$ tais que

$$
\Phi_{1} \circ f_{1}=g \circ \phi_{1} \text { e } \Phi_{2} \circ f_{2}=g \circ \phi_{2} .
$$

Sejam $\omega_{1}=\left(\Phi_{1}^{-1}\right)^{*} \omega$ e $\omega_{2}=\left(\Phi_{2}^{-1}\right)^{*} \omega$. Logo $\left[\omega_{1}\right]_{g}$ e $\left[\omega_{2}\right]_{g}$ são difeomorfas a $[\omega]_{f_{1}}$ e $\left[\omega_{2}\right]_{f_{2}}$, respectivamente. Seja $\rho:\left(\mathbb{R}^{2 n}, 0\right) \rightarrow\left(\mathbb{R}^{2 n}, 0\right)$ a simetria local de $g$ tal que

$$
\rho^{*}\left[\omega_{1}\right]_{g}=\left[\rho^{*} \omega_{1}\right]_{\rho^{-1} \circ g}=\left[\omega_{2}\right]_{g}
$$

Logo existe um germe de difeomorfismo $h:(\mathbb{R}, 0) \rightarrow(\mathbb{R}, 0)$ tal que $\rho \circ g=g \circ h$. Segue do Teorema A que existe um germe de difeomorfismo $H:\left(\mathbb{R}^{2 n}, 0\right) \rightarrow\left(\mathbb{R}^{2 n}, 0\right)$ tal que $\left.H\right|_{\operatorname{Im}(g)}=I d$ e $H^{*} \omega_{2}=\rho^{*} \omega_{1}$. Considere $T=\Phi_{2}^{-1} \circ H \circ \rho^{-1} \circ \Phi_{1}$. Logo 


$$
\begin{aligned}
& T^{*} \omega=\Phi_{1}^{*} \circ\left(\rho^{-1}\right)^{*} \circ H^{*} \circ\left(\Phi_{2}^{-1}\right)^{*} \omega=\Phi_{1}^{*} \circ\left(\rho^{-1}\right)^{*} \circ H^{*}\left(\omega_{2}\right)=\Phi_{1}^{*} \omega_{1}=\omega \mathrm{e} \\
& T\left(f_{1}\right)=\left(\Phi_{2}^{-1} \circ H \circ \rho^{-1} \circ \Phi_{1}\right)\left(f_{1}\right)=f_{2} \circ \phi_{2}^{-1} \circ h^{-1} \circ \phi_{1} .
\end{aligned}
$$

Logo os germes $f_{1}$ e $f_{2}$ são simplectomorfos.

Segue do Corolário 1.3.6 que a classificação simplética de germes de curvas $\mathcal{A}$-equivalentes a $g$ é obtida por meio da classificação das restrições algébricas a $g$ de formas simpléticas.

Teorema 1.3.7. ([D1]) O espaço vetorial das restrições algébricas a $g$ de 2-formas fechadas em $\left(\mathbb{R}^{2 n}, 0\right)$ tem dimensão finita e possui uma base formada por restrições algébricas a $g$ de 2-formas fechadas quase homogêneas.

Um resultado que auxilia encontrar uma base para o espaço vetorial das restrições algébricas a $g$ de 2-formas fechadas é o seguinte.

Proposição 1.3.8. ([D1]) Seja $a_{1}, \ldots, a_{k}$ uma base do espaço das restrições algébricas a $g$ de 2-formas satisfazendo as seguintes condições:

(1) $d a_{1}=\cdots=d a_{j}=0$;

(2) as restrições algébricas $d a_{j+1}, \ldots, d a_{k}$ são linearmente independentes.

Então $a_{1}, \ldots, a_{j}$ é uma base para o espaço das restrições algébricas a $g$ de 2-formas fechadas.

Proposição 1.3.9. ([D1]) Se os monômios $s(x)=\prod_{l=1}^{k} x_{l}^{s_{l}}$ e $p(x)=\prod_{l=1}^{k} x_{l}^{p_{l}}$ têm o mesmo quase grau então as 2-formas $s(x) d x_{i} \wedge d x_{j}$ e $p(x) d x_{i} \wedge d x_{j}$ tem mesma restrição algébrica a $g$.

Definição 1.3.10. Um germe de um campo de vetores $X$ em $\mathbb{R}^{m}$ é levantável sobre um germe de curva parametrizada $f:(\mathbb{R}, 0) \rightarrow\left(\mathbb{R}^{m}, 0\right)$ se existe um germe de função $h$ em $(\mathbb{R}, 0)$ tal que

$$
h\left(\frac{d f}{d t}\right)=X \circ f
$$

Definição 1.3.11. Seja a uma restrição algébrica a um germe de curva parametrizada $f$ de uma 2-forma em $\left(\mathbb{R}^{2 n}, 0\right)$. O espaço tangente a órbita de a é gerado por $\mathcal{L}_{X}$ a ao longo dos germes de campo de vetores levantáveis $X$ sobre $f$.

Como visto no Teorema 1.3.7, o espaço vetorial das restrições algébricas de 2-formas fechadas a $g$ tem dimensão finita. Seja $K(g)$ o menor inteiro positivo tal que se $a$ é uma restrição algébrica a $g$ de uma 2-forma fechada quase homogênea de quase grau $r>K(g)$ então $a=0$.

Teorema 1.3.12. ([D1]) Sejam $g(t)=\left(t^{\lambda_{1}}, \ldots, t^{\lambda_{k}}, 0, \ldots, 0\right)$ e $X_{s}$ um campo de vetores tal que $X_{s} \circ g=$ $t^{s+1} \mathrm{dg} / \mathrm{dt}$. O espaço tangente a órbita da restrição algébrica $a_{r}$ quase homogênea de quase grau $r$ é gerado por $\mathcal{L}_{X_{s}} a_{r}$ para valores de s que são combinações $\mathbb{Z}_{\geq 0}$-linear de $\lambda_{1}, \ldots, \lambda_{k}$ e menores que $K(g)-r$. 
O seguinte resultado fornece uma ferramenta para obter as órbitas das restrições algébricas a $g$ de formas simpléticas.

Teorema 1.3.13. ([D1]) Seja $a_{1}, \ldots, a_{p}$ uma base do espaço vetorial das restrições algébricas a g representados por 2-formas fechadas quase homogêneas de quase graus $\delta_{1} \leq \cdots \leq \delta_{s}<\delta_{s+1} \leq \cdots \leq \delta_{p}$. Seja $a=\sum_{j=s}^{p} c_{j} a_{j}$, onde $c_{j} \in \mathbb{R}$ para $j=s, \ldots, p$ e $c_{s} \neq 0$. Se existe um campo de vetores quase homogêneo $X$ levantável sobre $g$ tal que $\mathcal{L}_{X} a_{s}=r a_{k}$ para $k>$ s e $r \neq 0$ então a é difeomorfa a $\sum_{j=s}^{k-1} c_{j} a_{j}+\sum_{j=k+1}^{p} b_{j} a_{j}$ para algum $b_{j} \in \mathbb{R}, j=k+1, \ldots, p$.

\subsection{Invariantes}

Para distinguir as órbitas em sua classificação, Arnold em [A1] utilizou um invariante simplético específico para curvas parametrizadas que é a ordem de tangência lagrangiana. Em [DJZ2] os autores utilizaram os invariantes simpléticos: o índice de isotropia e a multiplicidade simplética. Estes invariantes foram suficientes para distinguir as órbitas nas classificações obtidas em [DJZ2] e em [D1]. No entanto eles não são suficientes para a classificação que pretendemos obter neste trabalho. Para tanto, introduzimos um invariante da ação das simetrias locais nas restrições algébricas a um germe de curva quase homogênea.

Sejam $\theta$ uma $k$-forma em $\left(\left(\mathbb{R}^{2 n}, \omega\right), 0\right)$ e $s$ o menor inteiro não negativo tal que $\theta^{(s)} \neq 0$. Dizemos que $s$ é a ordem de anulamento de $\theta$. Se $\theta^{(0)} \neq 0$ então a ordem de anulamento é nula. Se $\theta=0$ então a ordem de anulamento é $\infty$.

Definição 1.4.1. Seja $N$ um germe de um subconjunto do espaço simplético $\left(\left(\mathbb{R}^{2 n}, \omega\right), 0\right)$. O índice de isotropia de $N$ é a ordem máxima de anulamento de $\left.\omega\right|_{T M}$ onde $M$ é uma subvariedade que contém $N$.

O índice de isotropia pode ser obtido em termos das restrições algébricas de 2-formas a conjuntos quase homogêneos.

Teorema 1.4.2. ([DJZ2]) O indice de isotropia de um germe de subconjunto quase homogêneo $N$ no espaço simplético $\left(\left(\mathbb{R}^{2 n}, \omega\right), 0\right)$ é igual a ordem máxima de anulamento das 2-formas fechadas que representam a restrição algébrica $[\omega]_{N}$.

Definição 1.4.3. Sejam $N$ um germe de subconjunto de $\left(\left(\mathbb{R}^{2 n}, \omega\right), 0\right),(N)$ a órbita de $N$ com respeito

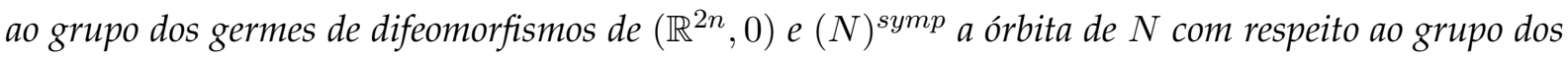
germes de simplectomorfismos de $\left(\left(\mathbb{R}^{2 n}, \omega\right), 0\right)$. A multiplicidade simplética de $N$ é a codimensão de

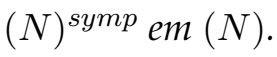

Teorema 1.4.4. ([DJZ2]) A multiplicidade simplética de um subconjunto quase homogêneo no espaço simplético $\left(\left(\mathbb{R}^{2 n}, \omega\right), 0\right)$ é igual a codimensão da órbita da restrição algébrica $[\omega]_{N}$ com respeito ao grupo das simetrias locais de $N$ no espaço vetorial das restrições algébricas das 2-formas fechadas a $N$.

Seja $L$ um germe de subvariedade lagrangiana em $\left(\left(\mathbb{R}^{2 n}, \omega\right), 0\right)$. Devido ao Exemplo 1.1.16, existem $H_{1}, \ldots, H_{n}$ germes de funções suaves de $\left(\mathbb{R}^{2 n}, 0\right)$ em $(\mathbb{R}, 0)$ tais que $L$ é definida por 
$H_{1}=\cdots=H_{n}=0$. A ordem de tangência de um germe de uma curva $g:(\mathbb{R}, 0) \rightarrow\left(\mathbb{R}^{2 n}, 0\right)$ a $L$ é a mínima ordem de anulamento dos germes de funções $H_{1} \circ g, \ldots, H_{n} \circ g$. Denotamos a ordem de tangência de $g$ a $L$ por $t(g, L)$.

Definição 1.4.5. A ordem de tangência lagrangiana $\operatorname{Lt}(g)$ de uma curva g é o máximo das $t(g, L)$ sobre todas as subvariedades lagrangianas $L$ do espaço simplético.

Este invariante simplético de curvas parametrizadas pode ser obtido em termos das restrições algébricas.

Proposição 1.4.6. ([D1]) Seja $g:(\mathbb{R}, 0) \rightarrow\left(\mathbb{R}^{2 n}, 0\right)$ um germe de uma curva parametrizada quase homogênea tal que a restrição algébrica de uma forma simplética a $g$ pode ser representada por uma 2forma fechada se anulando na origem. Então a ordem de tangência lagrangiana da curva g é a ordem máxima de anulamento em $g$ das 1-formas $\gamma$ tais que $[\omega]_{g}=[d \gamma]_{g}$.

A seguir definimos um invariante para distinguir as órbitas de restrições algébricas a germes de curvas parametrizadas quase homogêneas de $k$-formas sob a ação das simetrias locais. Seja $g:(\mathbb{R}, 0) \rightarrow\left(\mathbb{R}^{m}, 0\right)$ um germe de curva parametrizada quase homogênea definida por $g(t)=\left(t^{\lambda_{1}}, \ldots, t^{\lambda_{s}}, 0, \ldots, 0\right)$, onde $\lambda_{1}<\cdots<\lambda_{s}$ são inteiros positivos linearmente independentes sobre inteiros não negativos.

Definição 1.4.7. Sejam $a_{1}, a_{2}$ restrições algébricas a $g$ de $k$-formas em $\left(\mathbb{R}^{m}, 0\right)$. Sejam $r_{1}, r_{2}$ os mínimos inteiros não negativos tais que $a_{1}^{\left(r_{1}\right)} \neq 0$ e $a_{2}^{\left(r_{2}\right)} \neq 0$. Dizemos que $a_{1}$ e $a_{2}$ têm parte de quase grau mínimo proporcional se $r_{1}=r_{2}$ e

$$
a_{2}^{\left(r_{1}\right)}=c a_{1}^{\left(r_{1}\right)}, c \neq 0
$$

Proposição 1.4.8. Sejam $a_{1}$ e $a_{2}$ restrições algébricas a $g$ de $k$-formas em $\left(\mathbb{R}^{m}, 0\right)$. Se $a_{1}$ e $a_{2}$ são difeomorfas então elas têm parte de quase grau mínimo proporcional.

Demonstração. Podemos supor que $g(t)=\left(t^{\lambda_{1}}, \ldots, t^{\lambda_{s}}\right)$ devido às Proposições 1.2.11 e 1.2.12.

As restrições algébricas $a_{1}$ e $a_{2}$ são difeomorfas se existem germes de difeomorfismos $\Phi$ : $\left(\mathbb{R}^{s}, 0\right) \rightarrow\left(\mathbb{R}^{s}, 0\right)$ e $\phi:(\mathbb{R}, 0) \rightarrow(\mathbb{R}, 0)$ tais que $\Phi \circ g=g \circ \phi$ e $\Phi^{*} a_{1}=a_{2}$. Então

$$
\Phi\left(t^{\lambda_{1}}, \ldots, t^{\lambda_{s}}\right)=\left(\phi(t)^{\lambda_{1}}, \ldots, \phi(t)^{\lambda_{s}}\right)
$$

Como $\phi$ é um germe de difeomorfismo então é da forma $\phi(t)=c t+t^{2} \cdot u(t)$, onde $u$ é suave e $c \neq 0$. Então

$$
\Phi\left(t^{\lambda_{1}}, \ldots, t^{\lambda_{s}}\right)=\left(c^{\lambda_{1}} t^{\lambda_{1}}\left(1+\frac{t u(t)}{c}\right)^{\lambda_{1}}, \ldots, c^{\lambda_{s}} t^{\lambda_{s}}\left(1+\frac{t u(t)}{c}\right)^{\lambda_{s}}\right) .
$$


Como $\lambda_{1}, \ldots, \lambda_{s}$ são linearmente independentes sobre inteiros não negativos então $\Phi$ deve ser da forma

$\Phi(x)=\left(c^{\lambda_{1}} x_{1}+c_{1,2} x_{2}+\cdots+c_{1, s} x_{s}+\xi_{1}(x), c^{\lambda_{2}} x_{2}+c_{2,3} x_{3}+\cdots+c_{2, s} x_{s}+\xi_{2}(x), \cdots, c^{\lambda_{s}} x_{s}+\xi_{s}(x)\right)$,

onde $c_{i, j} \in \mathbb{R}$ e $\xi_{i} \in \mathcal{M}_{s}^{2}$.

Sejam $r_{1}, r_{2}$ os menores inteiros positivos tais que $a_{1}^{\left(r_{1}\right)} \neq 0$ e $a_{2}^{\left(r_{2}\right)} \neq 0$. Considere $\theta_{1}$ uma $k$-forma representante de $a_{1}$. A $k$-forma $\theta_{1}^{\left(r_{1}\right)}$ se escreve como

$$
\theta_{1}^{\left(r_{1}\right)}=\sum u_{I} x_{1}^{\gamma_{1}(I)} \ldots x_{s}^{\gamma_{s}(I)} d x_{i_{1}} \wedge \ldots \wedge d x_{i_{s}}
$$

onde $I=i_{1}, \ldots, i_{s}, u_{I} \in \mathbb{R}$ e $\gamma_{1}(I), \ldots, \gamma_{s}(I)$ são inteiros não negativos tais que $x_{1}^{\gamma_{1}(I)} \ldots x_{s}^{\gamma_{s}(I)}$ é um monômio de quase grau $r_{1}-\lambda_{i_{1}}-\cdots-\lambda_{i_{k}}$. Seja $\eta=\theta_{1}-\theta_{1}^{\left(r_{1}\right)}$. Temos que

$$
a_{2}=\Phi^{*} a_{1}=\Phi^{*}\left[\theta_{1}\right]_{g}=\left[\Phi^{*}\left(\theta_{1}^{\left(r_{1}\right)}+\eta\right)\right]_{g}=\left[\Phi^{*} \theta_{1}^{\left(r_{1}\right)}\right]_{g}+\left[\Phi^{*} \eta\right]_{g}
$$

Devido a lei de formação da $\Phi$ temos que $\left[\Phi^{*} \eta\right]_{g}^{(j)}=0$, para todo $j<r_{1}+1$. Denotemos as funções coordenadas de $\Phi$ por $\Phi_{1}, \ldots, \Phi_{s}$. Temos que

$$
\begin{aligned}
\Phi^{*} \theta_{1}^{\left(r_{1}\right)} & =\sum u_{I} \Phi_{1}^{\gamma_{1}(I)}(x) \ldots \Phi_{s}^{\gamma_{s}(I)}(x) d \Phi_{i_{1}} \wedge \ldots \wedge d \Phi_{i_{k}} \\
& =\sum u_{I}\left(c^{\lambda_{1}} x_{1}\right)^{\gamma_{1}(I)} \ldots\left(c^{\lambda_{s}} x_{s}\right)^{\gamma_{s}(I)} d\left(c^{\lambda_{i_{1}}} x_{i_{1}}\right) \wedge \ldots \wedge d\left(c^{\lambda_{i_{k}}} x_{i_{k}}\right)+\nu \\
& =\sum u_{I} c^{\left(\lambda_{1} \gamma_{1}(I)+\cdots+\lambda_{s} \gamma_{s}(I)\right)} c^{\lambda_{i_{1}}+\cdots+\lambda_{i_{k}}} x_{1}^{\gamma_{1}(I)} \ldots x_{s}^{\gamma_{s}(I)} d x_{i_{1}} \wedge \ldots \wedge d x_{i_{k}}+\nu \\
& =c^{r_{1}} \sum u_{I} x_{1}^{\gamma_{1}(I)} \cdots x_{s}^{\gamma_{s}(I)} d x_{i_{1}} \wedge \ldots \wedge d x_{i_{k}}+\nu \\
& =c^{r_{1}} \theta_{1}^{\left(r_{1}\right)}+\nu
\end{aligned}
$$

onde $\nu$ é uma $k$-forma tal que $\nu^{(j)}=0$, para todo $j<r_{1}+1$. Temos que

$$
a_{2}=\Phi^{*} a_{1}=\Phi^{*}\left[\theta_{1}\right]_{g}=c^{r_{1}}\left[\theta_{1}^{\left(r_{1}\right)}\right]_{g}+[\mu]_{g}=c^{r_{1}} a_{1}^{\left(r_{1}\right)}+[\mu]_{g},
$$

onde $\mu=\Phi^{*} \eta+\nu$. Portanto $r_{1}=r_{2}$ e $a_{2}^{\left(r_{1}\right)}=c^{r_{1}} a_{1}^{\left(r_{1}\right)}$. Logo $a_{1}$ e $a_{2}$ têm parte de quase grau mínimo proporcional. 


\section{- \\ Classificação simplética de curvas parametrizadas}

Este capítulo é dedicado à classificação simplética de germes de curvas com semigrupos $(4,5,6),(4,5,7)$ e $(4,5,6,7)$. Na primeira seção fazemos a classificação simplética de germes de curvas com semigrupo $(4,5,6,7)$. Nas seções 2 e 3 realizamos a classificação simplética de germes de curvas com semigrupo $(4,5,6)$ e $(4,5,7)$ utilizando um algoritmo desenvolvido no software Singular para determinar bases dos espaços vetoriais das restrições algébricas a germes de curvas definidas por $t \rightarrow\left(t^{4}, t^{5}, t^{6}\right)$ e $t \rightarrow\left(t^{4}, t^{5}, t^{7}\right)$. O algoritmo encontra-se no Capítulo 4.

Sejam $k$ um inteiro não negativo e $g:(\mathbb{R}, 0) \rightarrow\left(\mathbb{R}^{2 n}, 0\right)$ um germe de curva parametrizada. Fixaremos algumas notações que serão usadas ao longo do capítulo:

- $\left[\Lambda^{k}\left(\mathbb{R}^{2 n}\right)\right]_{g}$ : o espaço vetorial das restrições algébricas a $g$ de elementos em $\Lambda^{k}\left(\mathbb{R}^{2 n}\right)$.

- $\Lambda^{k, c l}\left(\mathbb{R}^{2 n}\right)$ : o subespaço vetorial de $\Lambda^{k}\left(\mathbb{R}^{2 n}\right)$ das $k$-formas fechadas em $\left(\mathbb{R}^{2 n}, 0\right)$.

- $\left[\Lambda^{k, c l}\left(\mathbb{R}^{2 n}\right)\right]_{g}$ : o subespaço vetorial de $\left[\Lambda^{k}\left(\mathbb{R}^{2 n}\right)\right]_{g}$ das restrições algébricas a $g$ de elementos $\operatorname{em~} \Lambda^{k, c l}\left(\mathbb{R}^{2 n}\right)$.

- $\left[\operatorname{Symp}\left(\mathbb{R}^{2 n}\right)\right]_{g}$ : o subconjunto de $\left[\Lambda^{2, c l}\left(\mathbb{R}^{2 n}\right)\right]_{g}$ das restrições algébricas a $g$ de elementos em $\operatorname{Symp}\left(\mathbb{R}^{2 n}\right)$.

Convenção: Até o final do capítulo consideramos o espaço euclidiano $\mathbb{R}^{2 n}$ com coordenadas $\left(x_{1}, \ldots, x_{2 n}\right)$.

\subsection{Classificação simplética de curvas com semigrupo $(4,5,6,7)$}

Esta seção é dedicada à demonstração do seguinte resultado. 
Teorema 2.1.1. (i) Considere o espaço simplético $\left(\mathbb{R}^{2 n}, \omega=\sum_{i=1}^{n} d x_{i} \wedge d x_{i+n}\right)$. Um germe de curva $f:(\mathbb{R}, 0) \rightarrow\left(\mathbb{R}^{2 n}, 0\right)$ com semigrupo $(4,5,6,7)$ é simplectomorfo a um, e somente um, dos germes de curvas na segunda coluna da Tabela 2.1 para $n=2$. Para o caso $n=3$ o germe $f$ é simplectomorfo a um, e somente um, dos germes de curvas nas linhas 1-14 da Tabela 2.2. Finalmente, quando $n \geq 4$ o germe $f$ é simplectomorfo a um, e somente um, dos germes de curvas da Tabela 2.2.

(ii) Os parâmetros $c, c_{1} e c_{2}$ são modais.

Tabela 2.1: Formas normais em $\mathbb{R}^{4}$

\begin{tabular}{llccc}
\hline & Formas normais de $f$ & $\mu_{\text {sympl }}(f)$ & $\iota(f)$ & $L t(f)$ \\
\hline 1 & $t \rightarrow\left(t^{4}, t^{5}+c_{1} t^{7}, t^{6}, c_{2} t^{7}\right), c_{2} \neq 0$ & 2 & 0 & 5 \\
2 & $t \rightarrow\left(t^{4}, \pm t^{6}+c_{1} t^{7}, t^{5}, c_{2} t^{7}\right), c_{1}, c_{2} \neq 0$ & 3 & 0 & 6 \\
3 & $t \rightarrow\left(t^{4}, \pm t^{6}, t^{7}, c_{1} t^{5}+c_{2} t^{6}\right), c_{1} \neq 0$ & 4 & 0 & 6 \\
4 & $t \rightarrow\left(t^{4}, c_{1} t^{7}, t^{6},-t^{5}+c_{2} t^{7}\right), c_{1} \neq 0,-\frac{3}{2},-\frac{1}{3},-1, \frac{12}{7}$ & 4 & 0 & 6 \\
5 & $t \rightarrow\left(t^{4},-\frac{3}{2} t^{7}, t^{5}, t^{6}+c t^{7}\right), c \neq 0$ & 4 & 0 & 6 \\
6 & $t \rightarrow\left(t^{4},-\frac{3}{2} t^{7}, t^{6}, c t^{7}-t^{5}\right)$ & 5 & 0 & 6 \\
7 & $t \rightarrow\left(t^{4},-t^{7}+c_{1} t^{10}, t^{6},-t^{5}+c_{2} t^{7}\right)$ & 5 & 0 & 6 \\
8 & $t \rightarrow\left(t^{4},-\frac{1}{3} t^{7}+c_{1} t^{11}, t^{6},-t^{5}+c_{2} t^{7}\right)$ & 5 & 0 & 6 \\
9 & $t \rightarrow\left(t^{4}, \frac{12}{7} t^{7}+c t^{9}, t^{5}, t^{6}\right)$ & 4 & 0 & 6 \\
\hline
\end{tabular}


Tabela 2.2: Formas normais em $\mathbb{R}^{2 n}, n>2$

\begin{tabular}{clccc}
\hline & Formas normais em $f$ & $\mu_{\text {sympl }}(f)$ & $\iota(f)$ & $L t(f)$ \\
\hline 1 & $t \rightarrow\left(t^{4}, t^{5}+c_{1} t^{7}, t^{6}, c_{2} t^{7}, t^{7}, 0, \ldots, 0\right)$ & 2 & 0 & 5 \\
2 & $t \rightarrow\left(t^{4}, \pm t^{6}+c_{1} t^{7}, t^{5}, c_{2} t^{7}, t^{7}, 0, \ldots, 0\right), c_{1} \neq 0$ & 3 & 0 & 6 \\
3 & $t \rightarrow\left(t^{4}, \pm t^{6}, t^{5}, c_{1} t^{7}, t^{6}, c_{2} t^{7}, 0, \ldots, 0\right)$ & 4 & 0 & 6 \\
4 & $t \rightarrow\left(t^{4}, c_{1} t^{7}, t^{5}, t^{6}, t^{7}, c_{2} t^{6}, 0, \ldots, 0\right), c_{1} \neq-\frac{3}{2},-\frac{1}{3},-1, \frac{12}{7}$ & 4 & 0 & 6 \\
5 & $t \rightarrow\left(t^{4},-\frac{3}{2} t^{7}, t^{5}, t^{6}+c t^{7}, 0, \ldots, 0\right), c \neq 0$ & 4 & 0 & 6 \\
6 & $t \rightarrow\left(t^{4},-\frac{3}{2} t^{7}, t^{6}, c t^{7}-t^{5}, 0, \ldots, 0\right)$ & 5 & 0 & 6 \\
7 & $t \rightarrow\left(t^{4},-t^{7}+c_{1} t^{10}, t^{6},-t^{5}+c_{2} t^{7}, 0, \ldots, 0\right)$ & 5 & 0 & 6 \\
8 & $t \rightarrow\left(t^{4},-\frac{1}{3} t^{7}+c_{1} t^{11}, t^{6},-t^{5}+c_{2} t^{7}, 0, \ldots, 0\right)$ & 5 & 0 & 6 \\
9 & $t \rightarrow\left(t^{4}, \frac{12}{7} t^{7}+c t^{9}, t^{5}, t^{6}, 0, \ldots, 0\right)$ & 4 & 0 & 6 \\
10 & $t \rightarrow\left(t^{4}, t^{7}, t^{5}, 0, t^{6}, c t^{7}, 0, \ldots, 0\right)$ & 4 & 0 & 7 \\
11 & $t \rightarrow\left(t^{4}, 0, t^{5}, \pm t^{7}, t^{6}, c t^{7}, 0, \ldots, 0\right)$ & 6 & 0 & 7 \\
12 & $t \rightarrow\left(t^{4}, c t^{9}, t^{5}, 0, t^{6}, t^{7}, 0, \ldots, 0\right), c \neq-1,-2$ & 7 & 0 & 7 \\
13 & $t \rightarrow\left(t^{4}, 2 t^{9}+c t^{10}, t^{5}, 0, t^{6}, t^{7}, 0, \ldots, 0\right)$ & 7 & 0 & 7 \\
14 & $t \rightarrow\left(t^{4},-t^{9}+c t^{11}, t^{5}, 0, t^{6}, t^{7}, 0, \ldots, 0\right)$ & 6 & 1 & 9 \\
15 & $t \rightarrow\left(t^{4}, t^{9}, t^{5}, 0, t^{6}, 0, t^{7}, 0, \ldots, 0\right)$ & 7 & 1 & 10 \\
16 & $t \rightarrow\left(t^{4}, \pm t^{10}, t^{5}, 0, t^{6}, 0, t^{7}, 0, \ldots, 0\right)$ & 8 & 1 & 11 \\
17 & $t \rightarrow\left(t^{4}, t^{11}, t^{5}, 0, t^{6}, 0, t^{7}, 0, \ldots, 0\right)$ & 9 & $\infty$ & $\infty$ \\
18 & $t \rightarrow\left(t^{4}, 0, t^{5}, 0, t^{6}, 0, t^{7}, 0, \ldots, 0\right)$ & & & \\
\hline
\end{tabular}

Aqui denotamos $\mu_{\text {sympl }}(f), \iota(f)$ e $\operatorname{Lt}(f)$ a multiplicidade simplética, índice de isotropia e ordem de tangência lagrangiana, respectivamente.

A demonstração do Teorema 2.1.1 será feita em etapas. De acordo com a Proposição 1.3.3, um germe de curva parametrizada quase homogênea em $\left(\mathbb{R}^{2 n}, 0\right)$ tem semigrupo $(4,5,6,7)$ se, e somente se, é $\mathcal{A}$-equivalente a $g:(\mathbb{R}, 0) \rightarrow\left(\mathbb{R}^{2 n}, 0\right)$ dado por $g(t)=\left(t^{4}, t^{5}, t^{6}, t^{7}, 0, \ldots, 0\right)$. Devido ao Corolário 1.3.6, para se obter a classificação dos germes de curvas parametrizadas com semigrupo $(4,5,6,7)$ sob a ação simplética é suficiente classificar restrições algébricas a $g$ de formas simpléticas sob a ação das simetrias locais de $g$. Devido ao Teorema 1.2.14, uma restrição algébrica a $g$ de uma 2-forma fechada tem um representante simplético mergulhado em $\mathbb{R}^{2 n_{0}}$, para algum $n_{0} \geq n$. A Proposição 2.1.4 fornece uma base para $\left[\Lambda^{2, c l}\left(\mathbb{R}^{2 n}\right)\right]_{g}$. Na Proposição 2.1.5 encontramos um conjunto finito de órbitas das restrições algébricas a $g$ de formas simpléticas. Finalmente utilizamos o Corolário 1.3.6 e a Proposição 2.1.5 para demonstrar o Teorema 2.1.1. 
Observação 2.1.2. Por definição, uma k-forma em $\mathscr{A}_{0}^{k}\left(\operatorname{Im}(g),\left(\mathbb{R}^{2 n}, 0\right)\right)$ é escrita como $\alpha+d \beta$, onde $\alpha$ é uma $k$-forma e $\beta$ é uma $(k-1)$-forma ambas identicamente nulas em $\operatorname{Im}(g)$. Se $\alpha+d \beta$ for quase homogênea de quase grau $r$ então $\alpha$ e $\beta$ se escrevem como

$$
\begin{aligned}
& \alpha(x)=\sum \alpha_{i_{1} \cdots i_{k}}(x) d x_{i_{1}} \wedge \cdots \wedge d x_{i_{k}} \\
& \beta(x)=\sum \beta_{j_{1} \cdots j_{k-1}}(x) d x_{j_{1}} \wedge \cdots \wedge d x_{j_{k-1}},
\end{aligned}
$$

onde $\alpha_{i_{1} \cdots i_{k}}$ e $\beta_{j_{1} \cdots j_{k-1}}$ são polinômios quase homogêneos de quase graus $r-\lambda_{i_{1}}-\cdots-\lambda_{i_{k}}$ er $r-\lambda_{j_{1}}-$ $\cdots-\lambda_{j_{k-1}}$, respectivamente. Por definição, $\alpha_{i_{1} \cdots i_{k}}$ e $\beta_{j_{1} \cdots j_{k-1}}$ se anulam em $\operatorname{Im}(g)$.

Seja $I(g)$ o ideal dos polinômios que se anulam em $\operatorname{Im}(g)$.

Proposição 2.1.3. Um conjunto de geradores de $I(g)$ é dado por

$$
\begin{aligned}
& g_{10}=x_{2}^{2}-x_{1} x_{3}, \quad g_{11}=x_{1} x_{4}-x_{2} x_{3}, \quad g_{12}^{+}=x_{1}^{3}-x_{3}^{2}, \\
& g_{12}^{-}=x_{1}^{3}-x_{2} x_{4}, \quad g_{13}=x_{1}^{2} x_{2}-x_{3} x_{4}, \quad g_{14}=x_{4}^{2}-x_{1}^{2} x_{3} .
\end{aligned}
$$

Demonstração. Os geradores de $I(g)$ são encontrados por meio de um algoritmo no software Singular. O algoritmo é apresentado com detalhes no Capítulo 4.

Devido ao Teorema 1.3.7, temos que a dimensão de $\left[\Lambda^{2, c l}\left(\mathbb{R}^{2 n}\right)\right]_{g}$ é finita. O próximo resultado fornece uma base.

Proposição 2.1.4. Uma base do espaço $\left[\Lambda^{2, c l}\left(\mathbb{R}^{2 n}\right)\right]_{g}$ é dada por:

$$
\begin{array}{lll}
a_{9}=\left[d x_{1} \wedge d x_{2}\right]_{g} & a_{10}=\left[d x_{1} \wedge d x_{3}\right]_{g} & a_{11}^{+}=\left[d x_{2} \wedge d x_{3}\right]_{g} \\
a_{11}^{-}=\left[d x_{1} \wedge d x_{4}\right]_{g} & a_{12}=\left[d x_{2} \wedge d x_{4}\right]_{g} & a_{13}^{+}=\left[d x_{3} \wedge d x_{4}\right]_{g} \\
a_{13}^{-}=\left[x_{1} d x_{1} \wedge d x_{2}\right]_{g} & a_{14}=\left[x_{1} d x_{1} \wedge d x_{3}\right]_{g} & a_{15}^{-}=\left[x_{1} d x_{1} \wedge d x_{4}\right]_{g}
\end{array}
$$

Demonstração. A próxima Tabela nos auxiliará a encontrar 2-formas com restrição algébrica a $g$ nula. 
Tabela 2.3: Geradores do ideal $I(g)$ e suas respectivas diferenciais

\begin{tabular}{|c|c|c|}
\hline quase grau $\delta$ & $g_{\delta}$ & Diferencial \\
\hline 10 & $g_{10}=x_{2}^{2}-x_{1} x_{3}$ & $d g_{10}=-x_{3} d x_{1}+2 x_{2} d x_{2}-x_{1} d x_{3}$ \\
\hline 11 & $g_{11}=x_{1} x_{4}-x_{2} x_{3}$ & $d g_{11}=x_{4} d x_{1}-x_{3} d x_{2}-x_{2} d x_{3}+x_{1} d x_{4}$ \\
\hline 12 & $g_{12}^{+}=x_{1}^{3}-x_{3}^{2}$ & $d g_{12}^{+}=3 x_{1}^{2} d x_{1}-2 x_{3} d x_{3}$ \\
\hline 12 & $g_{12}^{-}=x_{1}^{3}-x_{2} x_{4}$ & $d g_{12}^{-}=3 x_{1}^{2} d x_{1}-x_{4} d x_{2}-x_{2} d x_{4}$ \\
\hline 13 & $g_{13}=x_{1}^{2} x_{2}-x_{3} x_{4}$ & $d g_{13}=2 x_{1} x_{2} d x_{1}+x_{1}^{2} d x_{2}-x_{4} d x_{3}-x_{3} d x_{4}$ \\
\hline 14 & $g_{14}=x_{4}^{2}-x_{1}^{2} x_{3}$ & $d g_{14}=-2 x_{1} x_{3} d x_{1}-x_{1}^{2} d x_{3}+2 x_{4} d x_{4}$ \\
\hline
\end{tabular}

Como $\operatorname{Im}(g) \subset\left(\mathbb{R}^{4}, 0\right)$, então devido à Proposição 1.2.11 uma base do espaço vetorial $\left[\Lambda^{2, c l}\left(\mathbb{R}^{4}\right)\right]_{g}$ também é uma base do espaço vetorial $\left[\Lambda^{2, c l}\left(\mathbb{R}^{2 n}\right)\right]_{g}$.

Observe que restrições algébricas a $g$ de 2-formas quase homogêneas de quase graus diferentes são linearmente independentes devido à Proposição 1.2.16. Então podemos usar a graduação quase homogênea do espaço $\left[\Lambda^{2}\left(\mathbb{R}^{4}\right)\right]_{g}$ com respeito aos pesos $4,5,6$ e 7 para as variáveis $x_{1}, x_{2}, x_{3}$ e $x_{4}$, respectivamente. Ainda, devido à Tabela 2.3 e a Observação 2.1.2, uma 2-forma quase homogênea em $\mathscr{A}_{0}^{2}\left(\operatorname{Im}(g),\left(\mathbb{R}^{4}, 0\right)\right)$ tem quase grau no mínimo 14.

A Tabela 2.4 está organizada da seguinte forma: na primeira coluna é fixado um quase grau para os elementos da base descritos na segunda coluna. Os representantes das restrições algébricas são descritos na terceira coluna. Na quarta coluna são descritos as relações dos elementos de $\left[\Lambda^{2}\left(\mathbb{R}^{4}\right)\right]_{g}$ e suas respectivas demonstrações aparecem na coluna 5 .

Tabela 2.4: Base de $\left[\Lambda^{2, c l}\left(\mathbb{R}^{2 n}\right)\right]_{g}$

\begin{tabular}{|c|c|c|c|c|}
\hline$\delta$ & Base & Formas & Relações & Prova \\
\hline 9 & $a_{9}$ & $\alpha_{9}=d x_{1} \wedge d x_{2}$ & $a_{9}:=\left[\alpha_{9}\right]_{g}$ & \\
\hline 10 & $a_{10}$ & $\alpha_{10}=d x_{1} \wedge d x_{3}$ & $a_{10}:=\left[\alpha_{10}\right]_{g}$ & \\
\hline 11 & $a_{11}^{+}$ & $\alpha_{11}^{+}=d x_{2} \wedge d x_{3}$ & $a_{11}^{+}:=\left[\alpha_{11}^{+}\right]_{g}$ & \\
& $a_{11}^{-}$ & $\alpha_{11}^{-}=d x_{1} \wedge d x_{4}$ & $a_{11}^{-}:=\left[\alpha_{11}^{-}\right]_{g}$ & \\
\hline 12 & $a_{12}$ & $\alpha_{12}=d x_{2} \wedge d x_{4}$ & $a_{12}:=\left[\alpha_{12}\right]_{g}$ & \\
\hline 13 & $a_{13}^{+}$ & $\alpha_{13}^{+}=d x_{3} \wedge d x_{4}$ & $a_{13}^{+}=\left[\alpha_{13}^{-}\right]_{g}$ & \\
& $a_{13}^{-}$ & $x_{1} \alpha_{9}$ & $a_{13}^{-}=x_{1} a_{9}$ & \\
\hline 14 & $a_{14}$ & $x_{1} \alpha_{10}$ & $a_{14}=x_{1} a_{10}$ & \\
& & $x_{2} \alpha_{9}$ & $2 x_{2} a_{9}=a_{14}$ & {$\left[d x_{1} \wedge d g_{10}\right]$} \\
\hline 15 & $a_{15}^{+}$ & $x_{1} \alpha_{11}^{+}$ & $a_{15}^{+}=x_{1} a_{11}^{+}$ & \\
& $a_{15}^{-}$ & $x_{1} \alpha_{11}^{-}$ & $a_{15}^{-}=x_{1} a_{11}^{-}$ & \\
& & $x_{2} \alpha_{10}$ & $x_{2} a_{10}=a_{15}^{-}-a_{15}^{+}$ & {$\left[d x_{1} \wedge d g_{11}\right]_{g}$} \\
& & $x_{3} \alpha_{9}$ & $x_{3} a_{9}=a_{15}^{+}$ & {$\left[d x_{2} \wedge d g_{10}\right]_{g}$} \\
\hline \hline
\end{tabular}




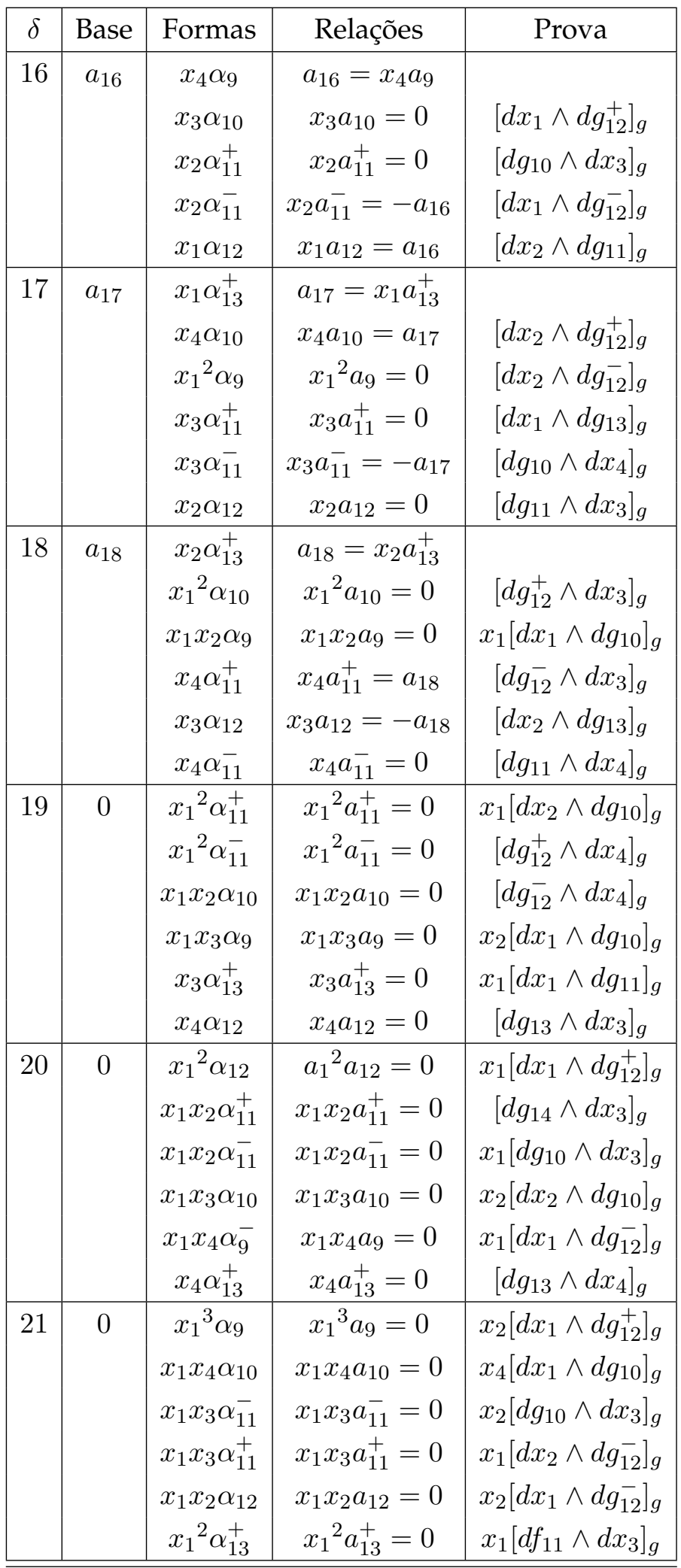




\begin{tabular}{|c|c|c|c|c|}
\hline$\delta$ & Base & Formas & Relações & Prova \\
\hline$\geq 22$ & 0 & $H_{1} \alpha_{9}$ & $H_{1} a_{9}=0$ & $H_{1} a_{9}=h_{1} x_{1}^{2} a_{9}=0$ \\
& & $H_{2} \alpha_{10}$ & $H_{2} a_{10}=0$ & $H_{2} a_{10}=h_{2} x_{3} a_{10}=0$ \\
& & $H_{3}^{+} \alpha_{11}^{+}$ & $H_{3}^{-} a_{11}^{+}=0$ & $H_{3}^{+} a_{11}^{+}=h_{3}^{+} x_{2} a_{11}^{+}=0$ \\
& & $H_{3}^{-} \alpha_{11}^{-}$ & $H_{3}^{-} a_{11}^{-}=0$ & $H_{3}^{-} a_{11}^{-}=h_{3}^{-} x_{4} a_{11}^{-}=0$ \\
& & $H_{4} \alpha_{12}$ & $H_{4} a_{12}=0$ & $H_{4} a_{12}=h_{4} x_{2} a_{12}=0$ \\
& $H_{5} \alpha_{13}^{+}$ & $H_{5} a_{13}^{+}=0$ & $1=22 x_{2}\left[d f_{10} \wedge d x_{4}\right]_{g}$ \\
& & & $1>22 H_{5} \alpha_{13}^{+}=h_{5} \tilde{a_{19}}=0$, \\
& & & onde $\tilde{a}_{19}$ é uma restrição algébrica de \\
& & & & 2-forma de quase grau 19 \\
\hline \hline
\end{tabular}

Começamos fixando o quase grau e encontramos os geradores com tal quase grau.

quase grau 9: Existe apenas uma 2-forma de quase grau 9, a saber $d x_{1} \wedge d x_{2}$. Logo o único gerador de quase grau 9 é $a_{9}:=\left[d x_{1} \wedge d x_{2}\right]_{g}$.

quase grau 10: Analogamente ao caso anterior, o único gerador de quase grau 10 é $a_{10}:=$ $\left[d x_{1} \wedge d x_{3}\right]_{g}$.

quase grau 11: Existem duas 2-formas de quase grau 11, a saber $d x_{2} \wedge d x_{3}$ e $d x_{1} \wedge d x_{4}$. Como não existe 2-forma de quase grau $11 \mathrm{em} \mathscr{A}_{0}^{2}\left(\operatorname{Im}(g),\left(\mathbb{R}^{4}, 0\right)\right)$, segue que os geradores de grau 11 são $a_{11}^{+}:=\left[d x_{2} \wedge d x_{3}\right]_{g}$ e $a_{11}^{-}:=\left[d x_{1} \wedge d x_{4}\right]_{g}$.

quase grau 12: Análogo aos casos 9 e 10, o único gerador de quase grau 12 é $a_{12}:=\left[d x_{2} \wedge d x_{4}\right]_{g}$.

quase grau 13: Análogo ao caso 11, os geradores de quase grau 13 são $a_{13}^{+}:=\left[d x_{3} \wedge d x_{4}\right]_{g} \mathrm{e}$ $a_{13}^{-}:=\left[x_{1} d x_{1} \wedge d x_{2}\right]_{g}$.

quase grau 14: Existem duas 2-formas de quase grau 14, a saber $x_{1} d x_{1} \wedge d x_{3}$ e $x_{2} d x_{1} \wedge d x_{2}$. Ambas têm mesma restrição algébrica a $g$. De fato, como $g_{10} \in I(g)$, segue da Observação 1.2.9 que $d x_{1} \wedge d g_{10} \in \mathscr{A}_{0}^{2}\left(\operatorname{Im}(g),\left(\mathbb{R}^{4}, 0\right)\right)$. Além disso,

$$
d x_{1} \wedge d g_{10}=2 x_{2} d x_{1} \wedge d x_{2}-x_{1} d x_{1} \wedge d x_{3} .
$$

Seja $a_{14}:=\left[x_{1} d x_{1} \wedge d x_{3}\right]_{g}$.

quase grau 15: Repetimos o processo descrito para o quase grau 14. Portanto os geradores são $a_{15}^{+}:=\left[x_{1} d x_{2} \wedge d x_{3}\right]_{g}$ e $a_{15}^{-}:=\left[x_{1} d x_{1} \wedge d x_{4}\right]_{g}$. 
Para os demais quase graus até 21 procedemos similarmente para obter seus geradores que são: $a_{16}:=\left[x_{4} d x_{1} \wedge d x_{2}\right]_{g}, a_{17}:=\left[x_{1} d x_{3} \wedge d x_{4}\right]_{g}$ e $a_{18}:=\left[x_{2} d x_{3} \wedge d x_{4}\right]_{g}$ (ver Tabela 2.4).

Provamos agora mais detalhadamente que as restrições algébricas a $g$ de 2-formas quase homogêneas com quase grau maior ou igual a 22 são nulas. Uma restrição algébrica a $g$ de uma 2-forma de quase grau $l \geq 22$ é combinação linear das restrições algébricas

$$
H_{1} a_{9}, H_{2} a_{10}, H_{3}^{+} a_{11}^{+}, H_{3}^{-} a_{11}^{-}, H_{4} a_{12} \text { e } H_{5} a_{13}^{+},
$$

onde $H_{i}$ é um polinômio quase homogêneo de quase grau $l-8-i, i \in\{1,2,4,5\}$, e $H_{3}^{+}, H_{3}^{-}$ são polinômios quase homogêneos de quase grau $l-11$.

Segue da Proposição 1.3.9 que existem $h_{1}, h_{2}$ e $h_{4}$ polinômios quase homogêneos de quase graus $l-17, l-16$ e $l-17$, respectivamente, e $h_{3}^{+}$e $h_{3}^{-}$polinômios quase homogêneos de quase graus $l-16$ e $l-18$, respectivamente, tais que

$$
\begin{aligned}
& H_{1} a_{9}=h_{1} x_{1}^{2} a_{9}, \quad H_{2} a_{10}=h_{2} x_{3} a_{10}, \\
& H_{3}^{+} a_{11}^{+}=h_{3}^{+} x_{2} a_{11}^{+}, \quad H_{3}^{-} a_{11}^{-}=h_{3}^{-} x_{4} a_{11}^{-}, \\
& H_{4} a_{12}=h_{4} x_{2} a_{12} .
\end{aligned}
$$

Como $x_{1}^{2} a_{9}=x_{3} a_{10}=x_{2} a_{11}^{+}=x_{4} a_{11}^{-}=x_{2} a_{12}=0$ (ver a Tabela 2.4) então

$$
H_{1} a_{9}=H_{2} a_{10}=H_{3}^{+} a_{11}^{+}=H_{3}^{-} a_{11}^{-}=H_{4} a_{12}=0 .
$$

A prova que $H_{5} a_{13}^{+}=0$ é dividida em 2 casos. Suponhamos que $l=22$. Devido à Proposição 1.3.9, podemos considerar, sem perda, que $H_{5}$ é um monômio quase homogêneo de quase grau 9 com coeficiente 1. Suponhamos que $H_{5}=x_{1} x_{2}$. De acordo com a Observação 1.2.9, $x_{2}\left[d g_{10} \wedge d x_{4}\right]_{g}=0$. Portanto

$$
\begin{aligned}
0 & =x_{2}\left[d g_{10} \wedge d x_{4}\right]_{g}=\left[-x_{2} x_{3} d x_{1} \wedge d x_{4}+2 x_{2}^{2} d x_{2} \wedge d x_{4}-x_{1} x_{2} d x_{3} \wedge d x_{4}\right]_{g} \\
& =\left[-x_{2} x_{3} d x_{1} \wedge d x_{4}+2 x_{2}^{2} d x_{2} \wedge d x_{4}\right]_{g}-\left[x_{1} x_{2} d x_{3} \wedge d x_{4}\right]_{g}
\end{aligned}
$$

Consequentemente

$$
H_{5} a_{13}^{+}=-x_{2} x_{3} a_{11}^{-}+2 x_{2}^{2} a_{12}=-x_{1} x_{4} a_{11}^{-}+2 x_{2}^{2} a_{12} .
$$


Como $x_{4} a_{11}^{-}=x_{2} a_{12}=0$ temos que $H_{5} a_{13}^{+}=0$.

Suponhamos que $l>22$. Observe que $H_{5} a_{13}^{+}$pode ser escrita como um produto de uma restrição algébrica a $g$ de uma 2-forma quase homogênea de quase grau 19 que possui restrição algébrica a $g$ nula (ver Tabela 2.4) e um polinômio quase homogêneo de quase grau $l-19$. Portanto $H_{5} a_{13}^{+}=0$.

Segue que $\left[\Lambda^{2}\left(\mathbb{R}^{4}\right)\right]_{g}$ é gerado por

$$
a_{9}, a_{10}, a_{11}^{+}, a_{11}^{-}, a_{12}, a_{13}^{+}, a_{13}^{-}, a_{14}, a_{15}^{-}, a_{15}^{+}, a_{16}, a_{17}, a_{18}
$$

Observe que $d a_{9}=d a_{10}=d a_{11}^{+}=d a_{11}^{-}=d a_{12}=d a_{13}^{+}=d a_{13}^{-}=d a_{14}=d a_{15}^{-}=0 \mathrm{e}$ que $d a_{15}^{+}, d a_{16}, d a_{17}, d a_{18}$ são linearmente independentes, devido à Proposição 1.2.16. Segue da Proposição 1.3.8 que $a_{9}, a_{10}, a_{11}^{+}, a_{11}^{-}, a_{12}, a_{13}^{+}, a_{13}^{-}, a_{14}, a_{15}^{-}$é uma base para o espaço vetorial $\left[\Lambda^{2, c l}\left(\mathbb{R}^{4}\right)\right]_{g}$.

Proposição 2.1.5. Seja a uma restrição algébrica a g de uma forma simplética em $\left(\mathbb{R}^{2 n}, 0\right)$, onde $n \geq 2$. Então a é difeomorfa a uma das restrições algébricas a $g$ da Tabela 2.5 quando $n=2$. Para o caso $n=3$ a é difeomorfa a uma das restrições algébricas a g nas linhas 1-14 da Tabela 2.6. Finalmente para o caso $n \geq 4$ a é difeomorfa a uma das restrições algébricas a $g$ da Tabela 2.6.

Tabela 2.5: Restrições algébricas em $\mathbb{R}^{4}$

$$
\begin{array}{|l|}
\left.\hline \omega_{1}\right]_{g}=a_{9}+c_{1} a_{11}^{-}+c_{2} a_{13}^{+}, \quad c_{2} \neq 0 \\
\left.\hline \omega_{2}\right]_{g}= \pm a_{10}+c_{1} a_{11}^{-}+c_{2} a_{12}, \quad c_{1}, c_{2} \neq 0 \\
{\left[\omega_{3}\right]_{g}= \pm a_{10}+c_{1} a_{12}+c_{2} a_{13}^{+}, \quad c_{1} \neq 0} \\
{\left[\omega_{4}\right]_{g}=a_{11}^{+}+c_{1} a_{11}^{-}+c_{2} a_{13}^{+}, \quad c_{1} \neq 0,-\frac{3}{2},-\frac{1}{3},-1, \frac{12}{7}} \\
{\left[\omega_{5}\right]_{g}=a_{11}^{+}-\frac{3}{2} a_{11}^{-}+c a_{12}, \quad c \neq 0} \\
{\left[\omega_{6}\right]_{g}=a_{11}^{+}-\frac{3}{2} a_{11}^{-}+c a_{13}^{+}} \\
{\left[\omega_{7}\right]_{g}=a_{11}^{+}-a_{11}^{-}+c_{1} a_{13}^{+}+c_{2} a_{14}} \\
{\left[\omega_{8}\right]_{g}=a_{11}^{+}-\frac{1}{3} a_{11}^{-}+c_{1} a_{13}^{+}+c_{2} a_{15}^{-}} \\
{\left[\omega_{9}\right]_{g}=a_{11}^{+}+\frac{12}{7} a_{11}^{-}+c a_{13}^{-}}
\end{array}
$$


Tabela 2.6: Restrições algébricas em $\mathbb{R}^{\geq 6}$

\begin{tabular}{|l|}
\hline$\left[\omega_{1}\right]_{g}=a_{9}+c_{1} a_{11}^{-}+c_{2} a_{13}^{+}$ \\
\hline$\left[\omega_{2}\right]_{g}= \pm a_{10}+c_{1} a_{11}^{-}+c_{2} a_{12}, c_{1} \neq 0$ \\
{$\left[\omega_{3}\right]_{g}= \pm a_{10}+c_{1} a_{12}+c_{2} a_{13}^{+}$} \\
\hline$\left[\omega_{4}\right]_{g}=a_{11}^{+}+c_{1} a_{11}^{-}+c_{2} a_{13}^{+}, \quad c_{1} \neq-\frac{3}{2},-\frac{1}{3},-1, \frac{12}{7}$ \\
{$\left[\omega_{5}\right]_{g}=a_{11}^{+}-\frac{3}{2} a_{11}^{-}+c a_{12}, \quad c \neq 0$} \\
{$\left[\omega_{6}\right]_{g}=a_{11}^{+}-\frac{3}{2} a_{11}^{-}+c a_{13}^{+}$} \\
{$\left[\omega_{7}\right]_{g}=a_{11}^{+}-a_{11}^{-}+c_{1} a_{13}^{+}+c_{2} a_{14}$} \\
{$\left[\omega_{8}\right]_{g}=a_{11}^{+}-\frac{1}{3} a_{11}^{-}+c_{1} a_{13}^{+}+c_{2} a_{15}^{-}$} \\
{$\left[\omega_{9}\right]_{g}=a_{11}^{+}+\frac{12}{7} a_{11}^{-}+c a_{13}^{-}$} \\
\hline$\left[\omega_{10}\right]_{g}=a_{11}^{-}+c a_{13}^{+}$ \\
\hline$\left[\omega_{11}\right]_{g}= \pm a_{12}+c a_{13}^{+}$ \\
\hline$\left[\omega_{12}\right]_{g}=a_{13}^{+}+c a_{13}^{-}, c \neq-1,2$ \\
{$\left[\omega_{13}\right]_{g}=a_{13}^{+}+2 a_{13}^{-}+c a_{14}$} \\
{$\left[\omega_{14}\right]_{g}=a_{13}^{+}-a_{13}^{-}+c a_{15}^{-}$} \\
{$\left[\omega_{15}\right]_{g}=a_{13}^{-}$} \\
\hline$\left[\omega_{16}\right]_{g}= \pm a_{14}$ \\
\hline$\left[\omega_{17}\right]_{g}=a_{15}^{-}$ \\
\hline$\left[\omega_{18}\right]_{g}=0$ \\
\hline \hline
\end{tabular}

Demonstração. A classificação é obtida por meio do Teorema 1.3.13. Devido às Proposições 1.2.18 e 2.1.4 é suficiente descrever os campos de vetores levantáveis sobre $g$ quase homogêneos de quase grau até 6.

$$
\begin{aligned}
& X_{0}=4 x_{1} \frac{\partial}{\partial x_{1}}+5 x_{2} \frac{\partial}{\partial x_{2}}+6 x_{3} \frac{\partial}{\partial x_{3}}+7 x_{4} \frac{\partial}{\partial x_{4}} \\
& X_{1}=4 x_{2} \frac{\partial}{\partial x_{1}}+5 x_{3} \frac{\partial}{\partial x_{2}}+6 x_{4} \frac{\partial}{\partial x_{3}}+7 x_{1}^{2} \frac{\partial}{\partial x_{4}} \\
& X_{2}=4 x_{3} \frac{\partial}{\partial x_{1}}+5 x_{4} \frac{\partial}{\partial x_{2}}+6 x_{1}^{2} \frac{\partial}{\partial x_{3}}+7 x_{1} x_{2} \frac{\partial}{\partial x_{4}} \\
& X_{3}=4 x_{4} \frac{\partial}{\partial x_{1}}+5 x_{1}^{2} \frac{\partial}{\partial x_{2}}+6 x_{1} x_{2} \frac{\partial}{\partial x_{3}}+7 m_{10} \frac{\partial}{\partial x_{4}} \\
& X_{4}=4 x_{1}^{2} \frac{\partial}{\partial x_{1}}+5 x_{1} x_{2} \frac{\partial}{\partial x_{2}}+6 m_{10} \frac{\partial}{\partial x_{3}}+7 m_{11} \frac{\partial}{\partial x_{4}} \\
& X_{5}=4 x_{1} x_{2} \frac{\partial}{\partial x_{1}}+5 m_{10} \frac{\partial}{\partial x_{2}}+6 m_{11} \frac{\partial}{\partial x_{3}}+7 m_{12} \frac{\partial}{\partial x_{4}} \\
& X_{6}=4 m_{10} \frac{\partial}{\partial x_{1}}+5 m_{11} \frac{\partial}{\partial x_{2}}+6 m_{12} \frac{\partial}{\partial x_{3}}+7 m_{13} \frac{\partial}{\partial x_{4}}
\end{aligned}
$$


onde $m_{i}$ é um monômio quase homogêneo de quase grau $i$. Na próxima Tabela calculamos a derivada de Lie de elementos da base de $\left[\Lambda^{2, c l}\left(\mathbb{R}^{2 n}\right)\right]_{g}$ como na Proposição 2.1.4 ao longo dos campos de vetores acima para $m_{10}=x_{1} x_{3}, m_{11}=x_{1} x_{4}, m_{12}=x_{1}^{3}$ e $m_{13}=x_{1}^{2} x_{2}$.

Tabela 2.7: Derivadas de Lie

\begin{tabular}{|c|cccc|}
\hline $\mathcal{L}_{X_{i}} a_{j}$ & $a_{9}$ & $a_{10}$ & $a_{11}^{+}$ & $a_{11}^{-}$ \\
\hline$X_{0}=E$ & $9 a_{9}$ & $10 a_{10}$ & $11 a_{11}^{+}$ & $11 a_{11}^{-}$ \\
$X_{1}$ & $5 a_{10}$ & $4 a_{11}^{+}+6 a_{11}^{-}$ & $6 a_{12}$ & $4 a_{12}$ \\
$X_{2}$ & $5 a_{11}^{-}-4 a_{11}^{+}$ & 0 & $-5 a_{13}^{+}-12 a_{13}^{-}$ & $4 a_{13}^{+}+7 a_{13}^{-}$ \\
$X_{3}$ & $-4 a_{12}$ & $-4 a_{13}^{+}+6 a_{13}^{-}$ & $7 a_{14}$ & $7 a_{14}$ \\
$X_{4}$ & $13 a_{13}^{-}$ & $14 a_{14}$ & $5 a_{15}^{-}$ & $15 a_{15}^{-}$ \\
$X_{5}$ & $7 a_{14}$ & $10 a_{15}^{-}$ & 0 & 0 \\
$X_{6}$ & $5 a_{15}^{-}$ & 0 & 0 & 0 \\
\hline
\end{tabular}

\begin{tabular}{|c|ccccc|}
\hline $\mathcal{L}_{X_{i}} a_{j}$ & $a_{12}$ & $a_{13}^{+}$ & $a_{13}^{-}$ & $a_{14}$ & $a_{15}^{-}$ \\
\hline$X_{0}$ & $12 a_{12}$ & $13 a_{13}^{+}$ & $13 a_{13}^{-}$ & $14 a_{14}$ & $15 a_{15}^{-}$ \\
$X_{1}$ & $5 a_{13}^{+}-14 a_{13}^{-}$ & $-14 a_{14}$ & $7 a_{14}$ & $10 a_{15}^{-}$ & 0 \\
$X_{2}$ & $-\frac{7}{2} a_{14}$ & $5 a_{15}^{-}$ & $5 a_{15}^{-}$ & 0 & 0 \\
$X_{3}$ & $10 a_{15}^{-}$ & 0 & 0 & 0 & 0 \\
$X_{4}$ & 0 & 0 & 0 & 0 & 0 \\
$X_{5}$ & 0 & 0 & 0 & 0 & 0 \\
$X_{6}$ & 0 & 0 & 0 & 0 & 0 \\
\hline
\end{tabular}

Devido às Proposições 1.2.14 e 2.1.4 toda restrição algébrica a $g$ representada por uma forma simplética é da forma:

$$
a=t_{1} a_{9}+t_{2} a_{10}+t_{3} a_{11}^{+}+t_{4} a_{11}^{-}+t_{5} a_{12}+t_{6} a_{13}^{+}+t_{7} a_{13}^{-}+t_{8} a_{14}+t_{9} a_{15}^{-}
$$

onde $t_{i} \in \mathbb{R}, i=1, \ldots, 9$.

Suponhamos que $t_{1} \neq 0$. Considere $\Psi_{1}:\left(\mathbb{R}^{4}, 0\right) \rightarrow\left(\mathbb{R}^{4}, 0\right)$ uma simetria local de $g$ definida por

$$
\Psi_{1}\left(x_{1}, x_{2}, x_{3}, x_{4}\right)=\left(t_{1}^{-\frac{4}{9}} x_{1}, t_{1}^{-\frac{5}{9}} x_{2}, t_{1}^{-\frac{6}{9}} x_{3}, t_{1}^{-\frac{7}{9}} x_{4}\right) .
$$

Temos que $\Psi_{1}{ }^{*} a=\frac{1}{t_{1}} a$. Sendo assim, podemos considerar que $t_{1}=1$. Como $\mathcal{L}_{X_{1}} a_{9}=5 a_{10}$, segue do Teorema 1.3.13 que $a$ é difeomorfa a $a_{9}+\tilde{t_{3}} a_{11}^{+}+\tilde{t_{4}} a_{11}^{-}+\tilde{t_{5}} a_{12}+\tilde{t_{6}} a_{13}^{+}+\tilde{t_{7}} a_{13}^{-}+\tilde{t_{8}} a_{14}+\tilde{t_{9}} a_{15}^{-}$, onde $\tilde{t}_{i} \in \mathbb{R}$, onde $i \in\{3, \ldots, 9\}$. Repetindo o processo concluímos que $a$ é difeomorfa a 
$a_{9}+c_{1} a_{11}^{-}+c_{2} a_{13}^{+}$, onde $c_{1}, c_{2} \in \mathbb{R}$.

A restrição algébrica $a_{9}+c_{1} a_{11}^{-}+c_{2} a_{13}^{+}$tem um representante simplético em $\left(\mathbb{R}^{4}, 0\right)$ se, e somente se, $c_{2} \neq 0$. De fato, $a_{9}+c_{1} a_{11}^{-}+c_{2} a_{13}^{+}$tem representante simplético em $\left(\mathbb{R}^{4}, 0\right)$ se, e somente se,

$$
\begin{aligned}
\sigma & =d x_{1} \wedge d x_{2}+c_{1} d x_{1} \wedge d x_{4}+c_{2} d x_{3} \wedge d x_{4} \\
& =d x_{1} \wedge\left(d x_{2}+c_{1} d x_{4}\right)+d x_{3} \wedge\left(c_{2} d x_{4}\right)
\end{aligned}
$$

é uma 2-forma simplética, pois se $\theta \in \mathscr{A}_{0}^{2}\left(\operatorname{Im}(g),\left(\mathbb{R}^{4}, 0\right)\right)$ então $\theta^{(j)}=0, j \leq 13$. Por outro lado, $\sigma$ é uma forma simplética em $\left(\mathbb{R}^{4}, 0\right)$ se, e somente se, $\sigma(0)$ é uma forma bilinear simplética. A matriz de $\sigma(0)$ dada por

$$
\left(\begin{array}{cccc}
0 & 1 & 0 & c_{1} \\
-1 & 0 & 0 & 0 \\
0 & 0 & 0 & c_{2} \\
-c_{1} & 0 & -c_{2} & 0
\end{array}\right)
$$

é invertível se, e somente se, $c_{2} \neq 0$.

A restrição algébrica $a_{9}+c_{1} a_{11}^{-}$tem um representante simplético em $\left(\mathbb{R}^{6}, 0\right)$. Uma 2-forma que representa $a_{9}+c_{1} a_{11}^{-}$é dada por

$$
d x_{1} \wedge d x_{2}+c_{1} d x_{1} \wedge d x_{4}
$$

De acordo com a Observação 1.2.9, $d x_{3} \wedge d x_{5}+d x_{4} \wedge d x_{6}$ tem restrição algébrica a $g$ nula, pois $x_{5}$ e $x_{6}$ se anulam em $\operatorname{Im}(g)$. Temos que

$$
\begin{aligned}
& d x_{1} \wedge d x_{2}+c_{1} d x_{1} \wedge d x_{4}+d x_{3} \wedge d x_{5}+d x_{4} \wedge d x_{6}= \\
& d x_{1} \wedge\left(d x_{2}+c_{1} d x_{4}\right)+d x_{3} \wedge d x_{5}+d x_{4} \wedge d x_{6}
\end{aligned}
$$

é um representante simplético de $a_{9}+c_{1} a_{11}^{-}$em $\left(\mathbb{R}^{6}, 0\right)$. Analogamente, um representante simplético de $a_{9}+c_{1} a_{11}^{-}$em $\left(\mathbb{R}^{2 n}, 0\right)$, para $n>3$, é

$$
d x_{1} \wedge\left(d x_{2}+c_{1} d x_{4}\right)+d x_{3} \wedge d x_{n+2}+d x_{4} \wedge d x_{n+3}+\cdots+d x_{n+1} \wedge d x_{2 n} .
$$

Portanto se $a$ tem um representante simplético em $\left(\mathbb{R}^{4}, 0\right)\left(c_{2} \neq 0\right)$, então $a$ é difeomorfa a $\left[\omega_{1}\right]_{g}$ da Tabela 2.5. Se $a$ tem um representante simplético em $\left(\mathbb{R}^{2 n}, 0\right)$, para $n \geq 3$, então $a$ é difeomorfa a $\left[\omega_{1}\right]_{g}$ da Tabela 2.6.

Suponha que $t_{1}=0$ e $t_{2} \neq 0$. Considere a simetria local de $g \Psi_{2}:\left(\mathbb{R}^{4}, 0\right) \rightarrow\left(\mathbb{R}^{4}, 0\right)$ definida 
por

$$
\Psi_{2}\left(x_{1}, x_{2}, x_{3}, x_{4}\right)=\left(\left|t_{2}\right|^{-\frac{4}{10}} x_{1},\left|t_{2}\right|^{-\frac{5}{10}} x_{2},\left|t_{2}\right|^{-\frac{6}{10}} x_{3},\left|t_{2}\right|^{-\frac{7}{10}} x_{4}\right) .
$$

Portanto podemos considerar que $t_{2}= \pm 1$.

Analogamente, $a$ é difeomorfa a $\pm a_{10}+c_{1} a_{11}^{-}+c_{2} a_{12}+c_{3} a_{13}^{+}$, onde $c_{1}, c_{2}, c_{3} \in \mathbb{R}$. Se $c_{1} \neq 0$ provaremos no Lema 2.1.6 que $a$ é difeomorfa a $\pm a_{10}+c_{1} a_{11}^{-}+c_{2} a_{12}$. Portanto $a$ é difeomorfa a $\left[\omega_{2}\right]_{g}$ ou $\left[\omega_{3}\right]_{g}$ da Tabela 2.5 se $a$ tiver um representante simplético em $\left(\mathbb{R}^{4}, 0\right)$, ou da Tabela 2.6 se $a$ tem um representante simplético em $\left(\mathbb{R}^{6}, 0\right)$.

Suponhamos que $t_{1}=\cdots=t_{i-1}=0$ e $t_{i} \neq 0$, onde $i \in\{4,5,7,8,9\}$. Seja $u$ inteiro positivo tal que $a^{(u)} \neq 0$ e $a^{(v)}=0$, para inteiro não negativo $v<u$. Consideremos as simetrias locais de $g \Psi_{i}:\left(\mathbb{R}^{4}, 0\right) \rightarrow\left(\mathbb{R}^{4}, 0\right)$ definidas por

$$
\begin{aligned}
& \Psi_{i}\left(x_{1}, x_{2}, x_{3}, x_{4}\right)=\left(t_{i}^{-\frac{4}{u}} x_{1}, t_{i}^{-\frac{5}{u}} x_{2}, t_{i}^{-\frac{6}{u}} x_{3}, t_{i}^{-\frac{7}{u}} x_{4}\right), \text { se } i \in\{4,7,9\} \text { e } \\
& \Psi_{i}\left(x_{1}, x_{2}, x_{3}, x_{4}\right)=\left(\left|t_{i}\right|^{-\frac{4}{u}} x_{1},\left|t_{i}\right|^{-\frac{5}{u}} x_{2},\left|t_{i}\right|^{-\frac{6}{u}} x_{3},\left|t_{i}\right|^{-\frac{7}{u}} x_{4}\right), \text { se } i \in\{5,8\} .
\end{aligned}
$$

Portanto podemos considerar $t_{i}=1$, se $i \in\{4,7,9\}$, ou $t_{i}= \pm 1$, se $i \in\{5,8\}$. Analogamente, se $a$ tem um representante simplético em $\left(\mathbb{R}^{6}, 0\right)$ então $a$ é difeomorfa a $\left[\omega_{10}\right]_{g}$ ou a $\left[\omega_{11}\right]_{g}$ da Tabela 2.6 e, se $a$ tem um representante simplético em $\left(\mathbb{R}^{2 n}, 0\right)$ para $n \geq 6$, então $a$ é difeomorfa a $\left[\omega_{l}\right]_{g}$ da Tabela $2.6, l \in\{10,11,15,16,17\}$.

Suponhamos que $t_{3} \neq 0$. Consideramos a simetria local de $g \Psi_{3}:\left(\mathbb{R}^{4}, 0\right) \rightarrow\left(\mathbb{R}^{4}, 0\right)$ dada por

$$
\Psi_{3}\left(x_{1}, x_{2}, x_{3}, x_{4}\right)=\left(t_{3}^{-\frac{4}{11}} x_{1}, t_{3}^{-\frac{5}{11}} x_{2}, t_{3}^{-\frac{6}{11}} x_{3}, t_{3}^{-\frac{7}{11}} x_{4}\right)
$$

Logo $a$ é difeomorfa a $\frac{1}{t_{3}} a$. Nesse caso, podemos supor que $t_{3}=1$. Vamos aplicar o Teorema 1.3.13 para a seguinte base de $\left[\Lambda^{2, c l}\left(\mathbb{R}^{4}\right)\right]_{g}$ :

$$
a_{9}, a_{10}, a_{11}^{-}, a_{11}^{+}+t_{4} a_{11}^{-}, a_{12}, a_{13}^{+}, a_{13}^{-}, a_{14}, a_{15}^{-} \text {. }
$$

Logo $a=a_{11}^{+}+t_{4} a_{11}^{-}+t_{5} a_{12}+t_{6} a_{13}^{+}+t_{7} a_{13}^{-}+t_{8} a_{14}+t_{9} a_{15}^{-}$é difeomorfa a uma das restrições algébricas a seguir:

(i) $a_{11}^{+}+c_{1} a_{11}^{-}+c_{2} a_{13}^{+}, \quad c_{1} \neq-\frac{3}{2},-1,-\frac{1}{3}, \frac{12}{7}$;

(ii) $a_{11}^{+}-\frac{3}{2} a_{11}^{-}+c a_{12} c \neq 0$;

(iii) $a_{11}^{+}-\frac{3}{2} a_{11}^{-}+c a_{13}^{+}$;

(iv) $a_{11}^{+}-a_{11}^{-}+c_{1} a_{13}^{+}+c_{2} a_{14}$; 
(v) $a_{11}^{+}-\frac{1}{3} a_{11}^{-}+c_{1} a_{13}^{+}+c_{2} a_{15}^{-}$;

(vi) $a_{11}^{+}+\frac{12}{7} a_{11}^{-}+c a_{13}^{-}$.

Vejamos os casos (ii) e (iii) mais detalhadamente. Suponhamos que $a$ seja da forma $a=$ $a_{11}^{+}-\frac{3}{2} a_{11}^{-}+t_{5} a_{12}+t_{6} a_{13}^{+}+t_{7} a_{13}^{-}+t_{8} a_{14}+t_{9} a_{15}^{-}$. De acordo com o Teorema 1.3.13, $a$ é difeomorfa a $a_{11}^{+}-\frac{3}{2} a_{11}^{-}+c_{1} a_{12}+c_{2} a_{13}^{+}, c_{1}, c_{2} \in \mathbb{R}$. Se $c_{1} \neq 0$, provaremos no Lema 2.1.6 que $a$ é difeomorfa a $a_{11}^{+}-\frac{3}{2} a_{11}^{-}+c a_{12}$.

Portanto $a$ é difeomorfa a $\left[\omega_{i}\right]_{g}, i=4, \ldots, 9$, da Tabela 2.5 , se $a$ tem um representante simplético em $\left(\mathbb{R}^{4}, 0\right)$, ou da Tabela 2.6 se $a$ tem representante simplético em $\left(\mathbb{R}^{2 n}, 0\right), n \geq 3$.

Quando $t_{6} \neq 0$ e $t_{i}=0, i<6$ seguimos os passos feitos quando $t_{1}=t_{2}=0$ e $t_{3} \neq 0$ para concluir que $a$ é difeomorfa a $\left[\omega_{i}\right]_{g}, i=12,13,14$ da Tabela 2.6 tendo representantes simpléticos em $\left(\mathbb{R}^{2 n}, 0\right), n \geq 6$. Por fim, quando $t_{i}=0$, para todo $i$, então $a=\left[\omega_{18}\right]_{g}$ da Tabela 2.6 tendo um representante simplético em $\left(\mathbb{R}^{2 n}, 0\right), n \geq 4$, dado por $d x_{1} \wedge d x_{n+1}+d x_{2} \wedge d x_{n+2}+\cdots+d x_{n} \wedge$ $d x_{2 n}$.

Lema 2.1.6. (i) A restrição algébrica $a=a_{11}^{+}-\frac{3}{2} a_{11}^{-}+c_{1} a_{12}+c_{2} a_{13}^{+}, c_{1} \neq 0$ é difeomorfa a $\left[\omega_{5}\right]_{g}$ das Tabelas 2.5 e 2.6 .

(ii) A restrição algébrica $\tilde{a}= \pm a_{10}+c_{1} a_{11}^{-}+c_{2} a_{12}+c_{3} a_{13}^{+}, c_{1} \neq 0$ é difeomorfa a $\left[\omega_{2}\right]_{g}$ das Tabelas 2.5 e 2.6.

Demonstração. (i) Se $c_{1} \neq 0$ então usamos o método de homotopia de Moser como em [D1] para mostrar que $a$ é difeomorfa a $\left[\omega_{5}\right]_{g}$ nas Tabelas 2.5 e 2.6. Seja $A_{t}=a_{11}^{+}-\frac{3}{2} a_{11}^{-}+$ $c_{1} a_{12}+(1-t) c_{2} a_{13}^{+}$, onde $t \in[0,1]$. Suponha que exista uma família de simetrias locais de $g \Phi_{t}:\left(\mathbb{R}^{4}, 0\right) \rightarrow\left(\mathbb{R}^{4}, 0\right), t \in[0,1]$ tal que

$$
\Phi_{t}^{*} A_{t}=a, \Phi_{0}=I d
$$

Então $A_{1}=\left[\omega_{5}\right]_{g}$ é difeomorfa a $a$. Considere $\eta_{t}$ o campo de vetores tal que $\frac{d}{d t} \Phi_{t}=\eta_{t} \circ \Phi_{t}$. De acordo com a Proposição 1.1.21, temos que

$$
0=\frac{d}{d t} \Phi_{t}{ }^{*} A_{t}=\Phi_{t}{ }^{*}\left(\mathcal{L}_{\eta_{t}} A_{t}-c_{2} a_{13}^{+}\right)
$$

Portanto

$$
\mathcal{L}_{\eta_{t}} A_{t}=c_{2} a_{13}^{+}
$$

Suponhamos que existam $b_{i}:[0,1] \rightarrow \mathbb{R}$ funções suaves, $i=1, \ldots, 4$, tais que 


$$
\eta_{t}=\sum_{i=1}^{4} b_{i}(t) X_{i}
$$

onde os $X_{i}$ 's são os campos de vetores da Tabela $2.7, i=1, \ldots, 4$. Temos que

$$
\begin{aligned}
c_{2} a_{13}^{+}= & {\left[5 c_{1} b_{1}(t)-11 b_{2}(t)\right] a_{13}^{+}+\left[-14 c_{1} b_{1}(t)-\frac{45}{2} b_{2}(t)\right] a_{13}^{-} } \\
+ & {\left[-14(1-t) c_{2} b_{1}(t)-\frac{7}{2} c_{1} b_{2}(t)-\frac{7}{2} b_{3}(t)\right] a_{14} } \\
& {\left[5(1-t) c_{2} b_{2}(t)+10 c_{1} b_{3}(t)-\frac{35}{2} b_{4}(t)\right] a_{15}^{-} . }
\end{aligned}
$$

Observe que o seguinte sistema

$$
\left\{\begin{array}{l}
5 c_{1} b_{1}(t)-11 b_{2}(t)=c_{2} \\
-14 c_{1} b_{1}(t)-\frac{45}{2} b_{2}(t)=0 \\
-14(1-t) c_{2} b_{1}(t)-\frac{7}{2} c_{1} b_{2}(t)-\frac{7}{2} b_{3}(t)=0 \\
5(1-t) c_{2} b_{2}(t)+10 c_{1} b_{3}(t)-\frac{35}{2} b_{4}(t)=0
\end{array}\right.
$$

claramente tem solução. Logo a família de difeomorfismos $\Phi_{t}$ associada a $\eta_{t}$ preserva $\operatorname{Im}(g)$, pois $\eta_{t}$ é levantável sobre $g$, e $\Phi_{t}{ }^{*} A_{t}=a$, para todo $t \in[0,1]$.

(ii) A prova é bem similar ao caso (i). Seja $\tilde{A}_{t}= \pm a_{10}+c_{1} a_{11}^{-}+c_{2} a_{12}+(1-t) c_{3} a_{13}^{+}$, onde $t \in[0,1]$. Suponha que existe $\Psi_{t}:\left(\mathbb{R}^{4}, 0\right) \rightarrow\left(\mathbb{R}^{4}, 0\right)$ uma família de simetrias locais de $g$ tal que para todo $t \in[0,1]$ tem-se

$$
\Psi_{t}^{*} \tilde{A}_{t}=\tilde{a}, \quad \Psi_{0}=I d
$$

Considere $\epsilon_{t}$ o campo de vetores tal que $\frac{d}{d t} \Psi_{t}=\epsilon_{t} \circ \Psi_{t}$. Diferenciando (2.2) ao longo de $t$ obtemos

$$
\mathcal{L}_{\epsilon} \tilde{A}_{t}=c_{3} a_{13}^{+} .
$$

Procuramos um campo de vetores da forma $\epsilon_{t}=\sum_{i=2}^{5} h_{i}(t) X_{i}$, onde $h_{i}:[0,1] \rightarrow \mathbb{R}$ são funções suaves, $i=2, \ldots, 5$, e os $X_{i}^{\prime} s$ são campos de vetores da Tabela 2.7. Portanto 


$$
\begin{aligned}
c_{3} a_{13}^{+}= & {\left[4 c_{1} h_{2}(t) \mp 4 h_{3}(t)\right] a_{13}^{+}+\left[7 c_{1} h_{2}(t) \pm 6 h_{3}(t)\right] a_{13}^{-} } \\
& +\left[-\frac{7}{2} h_{2}(t)+7 c_{1} h_{3}(t) \pm 14 h_{4}(t)\right] a_{14} \\
& {\left[5(1-t) c_{3} h_{2}(t)+10 c_{2} h_{3}(t)+15 c_{1} h_{4}(t) \pm 10 h_{5}(t)\right] a_{15}^{-} . }
\end{aligned}
$$

Observe que o sistema

$$
\left\{\begin{array}{l}
4 c_{1} h_{2}(t) \mp 4 h_{3}(t)=c_{3} \\
7 c_{1} h_{2}(t) \pm 6 h_{3}(t)=0 \\
-\frac{7}{2} c_{2} h_{2}(t)+7 c_{1} h_{3}(t) \pm 14 h_{4}(t)=0 \\
5(1-t) c_{3} h_{2}(t)+10 c_{2} h_{3}(t)+15 c_{1} h_{4}(t) \pm 10 h_{5}(t)=0
\end{array}\right.
$$

admite solução. Sendo assim, a família de difeomorfismos $\Psi_{t}$ associada a $\epsilon_{t}$ preserva $\operatorname{Im}(g)$ pois $\epsilon_{t}$ é levantável sobre $g$ e $\Psi_{t}{ }^{*} \tilde{A}_{t}=\tilde{a}$, para todo $t \in[0,1]$.

\section{Prova do Teorema 2.1.1}

Temos que $f$ é $\mathcal{A}$-equivalente a $g(t)=\left(t^{4}, t^{5}, t^{6}, t^{7}, 0, \ldots, 0\right)$. Sejam $\Phi:\left(\mathbb{R}^{2 n}, 0\right) \rightarrow\left(\mathbb{R}^{2 n}, 0\right) \mathrm{e}$ $\phi:(\mathbb{R}, 0) \rightarrow(\mathbb{R}, 0)$ germes de difeomorfismos tais que $\Phi \circ g=f \circ \phi$. Considere $\sigma=\Phi^{*} \omega$. Logo

$$
\Phi^{*}[\omega]_{f}=\left[\Phi^{*} \omega\right]_{\Phi^{-1} \circ f}=[\sigma]_{g \circ \phi^{-1}}=[\sigma]_{g}
$$

Devido à Proposição 2.1.5, a restrição algébrica $[\sigma]_{g}$ é difeomorfa a uma das restrições algébricas a $g$ de uma das Tabelas 2.5 ou 2.6, digamos $\left[\omega_{i}\right]_{g}$. Seja $\rho_{i}:\left(\mathbb{R}^{2 n}, 0\right) \rightarrow\left(\mathbb{R}^{2 n}, 0\right)$ a simetria local de $g$ tal que

$$
\rho_{i}^{*}[\sigma]_{g}=\left[\omega_{i}\right]_{g}
$$

O Teorema 1.1.18 garante a existência de um germe de difeomorfismo $\Psi_{i}:\left(\mathbb{R}^{2 n}, 0\right) \rightarrow$ $\left(\mathbb{R}^{2 n}, 0\right)$ tal que $\Psi_{i}^{*} \omega_{i}=\omega$. Portanto

$$
\left(\Phi \circ \rho_{i} \circ \Psi_{i}\right)^{*}[\omega]_{f}=\Psi_{i}^{*} \circ \rho_{i}^{*} \circ \Phi^{*}[\omega]_{f}=[\omega]_{\Psi_{i}^{-1} \circ g}
$$

ou seja, $[\omega]_{f}$ e $\left[\omega_{i}\right]_{\Psi_{i}^{-1} g}$ são difeomorfas. Segue do Corolário 1.3.6 que $f$ e $\Psi_{i}^{-1} \circ g$ são simplectomorofos. 
Para obter as Tabelas 2.1 e 2.2 utilizamos as Tabelas 2.5 e 2.6. Para fixar ideias, obtemos a primeira linha da Tabela 2.1 com mais detalhes.

A restrição algébrica $\left[\omega_{1}\right]_{g}$ da Tabela 2.5 é representada por

$$
\omega_{1}=d x_{1} \wedge d x_{2}+c_{1} d x_{1} \wedge d x_{4}+c_{2} d x_{3} \wedge d x_{4}=d x_{1} \wedge\left(d x_{2}+c_{1} d x_{4}\right)+d x_{3} \wedge\left(c_{2} d x_{4}\right),
$$

onde $c_{1}, c_{2} \in \mathbb{R} \operatorname{com} c_{2} \neq 0$.

Considere $F_{1}:\left(\mathbb{R}^{4}, 0\right) \rightarrow\left(\mathbb{R}^{4}, 0\right)$ definido por $F_{1}(x)=\left(x_{1}, x_{2}+c_{1} x_{4}, x_{3}, c_{2} x_{4}\right)$. Seja $\Psi_{1}=$ $F_{1}^{-1}$. Observe que $\Psi_{1}^{*} \omega_{1}=\omega$. A curva descrita na primeira linha da Tabela 2.1 é dada por

$$
f_{1}:=\left(\Psi_{1}^{-1} \circ g\right)(t)=\left(F_{1} \circ g\right)(t)=F_{1}\left(t^{4}, t^{5}, t^{6}, t^{7}\right)=\left(t^{4}, t^{5}+c_{1} t^{7}, t^{6}, c_{2} t^{7}\right) .
$$

A seguir calculamos os invariantes de $f_{1}$.

O espaço tangente à órbita de $\left[\omega_{1}\right]_{g}$ é gerado por $a_{9}, a_{10}, 5 a_{11}^{-}-4 a_{11}^{+}, a_{12}, a_{13}^{-}, a_{14}, a_{15}^{-}$. Portanto a codimensão do espaço tangente à órbita de $\left[\omega_{1}\right]_{g}$ com respeito ao grupo das simetrias locais de $g$ é 2. Segue do Teorema 1.4.4 que a multiplicidade simplética do germe de curva $f_{1}$ é 2 .

A ordem de anulamento de $\omega_{1}$ é 0 , pois $\omega_{1}^{(0)} \neq 0$. Segue do Teorema 1.4.2 que o índice de isotropia de $f_{1}$ é 0 .

Para facilitar o cálculo da ordem de tangência lagrangiana, reescrevemos as coordenadas $\left(x_{1}, x_{2}, x_{3}, x_{4}\right)$ de $\mathbb{R}^{4}$ como $\left(p_{1}, q_{1}, p_{2}, q_{2}\right)$ e, consequentemente, a forma simplética $\omega$ se escreve como $\omega=d p_{1} \wedge d q_{1}+d p_{2} \wedge d q_{2}$. Seja $L$ um germe de subvariedade lagrangiana em $\left(\left(\mathbb{R}^{4}, \omega\right), 0\right)$. De acordo com o Exemplo 1.1.16, existem subconjuntos disjuntos $I, J \subset\{1,2\}$ satisfazendo $I \cup J=\{1,2\}$ e $S:\left(\mathbb{R}^{2}, 0\right) \rightarrow(\mathbb{R}, 0)$ germe de função suave tais que $L$ é definida pelas funções

$$
\left\{\begin{array}{l}
l_{I}:=p_{I}+\frac{\partial S}{\partial q_{I}}\left(p_{I}, q_{J}\right) \\
l_{J}:=q_{J}-\frac{\partial S}{\partial p_{J}}\left(p_{I}, q_{J}\right) .
\end{array}\right.
$$

Temos que $t(g, L)$ é dada pela menor ordem de anulamento entre $l_{I} \circ f_{1}$ e $l_{J} \circ f_{1}$. Considerando todas as possibilidades para os subconjuntos $I, J$ concluímos que a ordem de tangência lagrangiana de $f_{1}$ é 5 .

Nas Tabelas 2.1 e 2.2 podemos observar que alguns germes de curvas possuem os mesmos invariantes simpléticos. Por exemplo, os germes de curvas nas linhas 3 e 4 da Tabela 2.1. Devido ao Corolário 1.3.6, os germes de curvas das linhas 3 e 4 são simplectomorfos se, e somente se, as restrições algébricas $\left[\omega_{3}\right]_{g}$ e $\left[\omega_{4}\right]_{g}$ da Tabela 2.5 são difeomorfas. Observe que $\left[\omega_{3}\right]_{g}$ e $\left[\omega_{4}\right]_{g}$ não têm parte de quase grau mínimo proporcional. Segue da Proposição 1.4 .8 que $\left[\omega_{3}\right]_{g}$ e $\left[\omega_{4}\right]_{g}$ 
não são difeomorfas e, portanto, os germes de curvas 3 e 4 da Tabela 2.1 não são simplectomorfos. Analogamente, devido à Proposição 1.4.8 e ao Corolário 1.3.6, as demais órbitas dos germes de curvas das Tabelas 2.1 e 2.2 que possuem mesmos invariantes simpléticos são disjuntas.

Por fim mostramos que os coeficientes $c, c_{1}$ e $c_{2}$ são modais. A prova em cada caso é bem similar. Para fixar ideias, provamos que $c_{1}$ e $c_{2}$ são modais na órbita de $\left[\omega_{2}\right]_{g}= \pm a_{10}+c_{1} a_{11}^{-}+$ $c_{2} a_{12}, c_{1} \neq 0$ da Tabela 2.2. O espaço tangente à órbita de $\left[\omega_{2}\right]_{g}$ é gerado pelas restrições algébricas $a_{15}^{-}, a_{14}, a_{13}^{+}, a_{13}^{-}, \pm\left(4 a_{11}^{+}+6 a_{11}^{-}\right)+4 c_{1} a_{12}, \pm 10 a_{10}+11 c_{1} a_{11}^{-}+12 c_{2} a_{12}$. Observe que a interseção do subespaço vetorial gerado por $a_{11}^{-}$e $a_{12}$ com o espaço tangente à órbita de $\left[\omega_{2}\right]_{g}$ é $\{0\}$. Portanto $c_{1}$ e $c_{2}$ são modais.

\subsection{Classificação simplética de curvas com semigrupo $(4,5,6)$}

Nesta seção classificamos germes de curvas parametrizadas com semigrupo $(4,5,6)$. Seguimos os passos descritos na seção anterior e utilizamos o software Singular para alguns cálculos (Veja Capítulo 4).

Teorema 2.2.1. (i) Considere o espaço simplético $\left(\mathbb{R}^{2 n}, \omega=\sum d x_{i} \wedge d x_{n+i}\right)$. Um germe de curva $f:(\mathbb{R}, 0) \rightarrow\left(\mathbb{R}^{2 n}, 0\right)$ com semigrupo $(4,5,6)$ é simplectomorfo a um, e somente um, dos germes de curvas nas linhas 1-3 da segunda coluna da Tabela 2.8 para $n=2$. Para o caso $n>2 f$ é simplectomorfo a um, e somente um, dos germes de curvas da segunda coluna da Tabela 2.8.

(ii) Os parâmetros $c, c_{1}, c_{2}$ são modais.

Tabela 2.8: Formas normais em $\mathbb{R}^{2 n}$

\begin{tabular}{clccc}
\hline & Formas normais de $f$ & $\mu_{\text {sympl }}(f)$ & $\iota(f)$ & $L t(f)$ \\
\hline 1 & $t \rightarrow\left(t^{4}, t^{5}+c_{1} t^{6}, t^{6}, c_{2} t^{5}, 0, \ldots, 0\right)$ & 2 & 0 & 5 \\
2 & $t \rightarrow\left(t^{4}, \pm t^{6}+c_{1} t^{9}, t^{5}, c_{2} t^{6}, 0 \ldots, 0\right), c_{1} \neq 0$ & 3 & 0 & 6 \\
3 & $t \rightarrow\left(t^{4}, \pm t^{6}+c_{1} t^{9}, t^{5}, c_{2} t^{12}, 0, \ldots, 0\right)$ & 4 & 0 & 6 \\
4 & $t \rightarrow\left(t^{4}, c_{1} t^{9}+c_{2} t^{10}, t^{5}, t^{6}, 0, \ldots, 0\right)$ & 4 & 0 & 6 \\
5 & $t \rightarrow\left(t^{4}, t^{9}+c_{1} t^{10}, t^{5}, 0, t^{6}, c_{2} t^{9}, 0, \ldots, 0\right)$ & 5 & 1 & 9 \\
6 & $t \rightarrow\left(t^{4}, \pm t^{10}, t^{5}, c_{1} t^{12}, t^{6}, c_{2} t^{9}, 0, \ldots, 0\right)$ & 6 & 1 & 10 \\
7 & $t \rightarrow\left(t^{4}, 0, t^{5}, 0, t^{6},-t^{9}+c t^{11}, 0, \ldots, 0\right)$ & 6 & 1 & 11 \\
8 & $t \rightarrow\left(t^{4}, 0, t^{5}, c t^{14}, t^{6},-t^{11}, 0, \ldots, 0\right)$ & 7 & 1 & 13 \\
9 & $t \rightarrow\left(t^{4}, 0, t^{5},-t^{14}, t^{6}, 0, \ldots, 0\right)$ & 7 & 2 & 15 \\
10 & $t \rightarrow\left(t^{4}, 0, t^{5}, 0, t^{6}, 0, \ldots, 0\right)$ & 8 & $\infty$ & $\infty$ \\
\hline
\end{tabular}

Seja $g:(\mathbb{R}, 0) \rightarrow\left(\mathbb{R}^{2 n}, 0\right)$ dado por $g(t)=\left(t^{4}, t^{5}, t^{6}, 0, \ldots, 0\right)$. Seja $I(g)$ o ideal dos polinômios que se anulam em $\operatorname{Im}(g)$. 
Proposição 2.2.2. Um conjunto de geradores de $I(g)$ é dado por

$$
g_{10}=x_{2}^{2}-x_{1} x_{3} \quad g_{12}=x_{1}^{3}-x_{3}^{2}
$$

Demonstração. Os geradores do ideal de polinômios que se anulam em $\operatorname{Im}(g)$ são encontrados por meio de um algoritmo no software Singular. O algoritmo é apresentado com detalhes no Capítulo 4.

Proposição 2.2.3. Uma base do espaço $\left[\Lambda^{2, c l}\left(\mathbb{R}^{2 n}\right)\right]_{g}$ é dada por:

$$
\begin{array}{cc}
a_{9}=\left[d x_{1} \wedge d x_{2}\right]_{g} & a_{10}=\left[d x_{1} \wedge d x_{3}\right]_{g} \\
a_{11}=\left[d x_{2} \wedge d x_{3}\right]_{g} & a_{13}=\left[x_{1} d x_{1} \wedge d x_{2}\right] \\
a_{14}=\left[x_{1} d x_{1} \wedge d x_{3}\right]_{g} & a_{15}=\left[x_{2} d x_{1} \wedge d x_{3}+x_{1} d x_{2} \wedge d x_{3}\right]_{g} \\
a_{17}=\left[x_{3} d x_{2} \wedge d x_{3}\right]_{g} & a_{19}=\left[x_{2}^{2} d x_{1} \wedge d x_{2}\right]_{g}
\end{array}
$$

Demonstração. Utilizamos um algoritmo no software Singular para determinar uma base para $\left[\Lambda^{2, c l}\left(\mathbb{R}^{2 n}\right)\right]_{g}$. O algoritmo encontra-se no Capítulo 4 .

Proposição 2.2.4. Seja a uma restrição algébrica a g de uma forma simplética em $\left(\mathbb{R}^{2 n}, 0\right)$, onde $n \geq 2$. Então a é difeomorfa a uma das restrições algébricas a g das linhas 1-3 da Tabela 2.9, para $n=2$. Se $n \geq 3$ então a é difeomorfa a uma das restrições algébricas a $g$ da Tabela 2.9.

Tabela 2.9: Restrições algébricas em $\mathbb{R}^{2 n}$

\begin{tabular}{|l|}
\hline$\left[\omega_{1}\right]_{g}=a_{9}+c_{1} a_{10}+c_{2} a_{11}$ \\
\hline$\left[\omega_{2}\right]_{g}= \pm a_{10}+c_{1} a_{11}+c_{2} a_{13}, \quad c_{1} \neq 0$ \\
{$\left[\omega_{3}\right]_{g}= \pm a_{10}+c_{1} a_{13}+c_{2} a_{17}$} \\
\hline$\left[\omega_{4}\right]_{g}=a_{11}+c_{1} a_{13}+c_{2} a_{14}$ \\
\hline$\left[\omega_{5}\right]_{g}=a_{13}+c_{1} a_{14}+c_{2} a_{15}$ \\
\hline$\left[\omega_{6}\right]_{g}= \pm a_{14}+c_{1} a_{15}+c_{2} a_{17}$ \\
\hline$\left[\omega_{7}\right]_{g}=a_{15}+c a_{17}$ \\
\hline$\left[\omega_{8}\right]_{g}=a_{17}+c a_{19}$ \\
\hline$\left[\omega_{9}\right]_{g}=a_{19}$ \\
\hline$\left[\omega_{10}\right]_{g}=0$ \\
\hline
\end{tabular}


Demonstração. Utilizamos basicamente o Teorema 1.3.13. Devido às Proposições 1.2.18 e 2.2.3 é suficiente descrever os campos de vetores levantáveis sobre $g$ quase homogêneos de quase grau até 10. Consideramos

$$
\begin{array}{ll}
X_{0}=E=4 x_{1} \frac{\partial}{\partial x_{1}}+5 x_{2} \frac{\partial}{\partial x_{2}}+6 x_{3} \frac{\partial}{\partial x_{3}} & X_{4}=x_{1} E \\
X_{5}=x_{2} E & X_{6}=x_{3} E \\
X_{7}=4 x_{2} x_{3} \frac{\partial}{\partial x_{1}}+5 x_{3}^{2} \frac{\partial}{\partial x_{2}}+6 x_{1}^{2} x_{2} \frac{\partial}{\partial x_{3}} & X_{8}=x_{1}^{2} E \\
X_{9}=x_{1} x_{2} E & X_{10}=x_{1} x_{3} E
\end{array}
$$

As derivadas de Lie dos elementos da base de $\left[\Lambda^{2, c l}\left(\mathbb{R}^{2 n}\right)\right]_{g}$ ao longo dos campos de vetores acima são descritos na Tabela 2.10.

Tabela 2.10: Derivadas de Lie

\begin{tabular}{|c|cccccccc|}
\hline $\mathcal{L}_{X_{i}} a_{j}$ & $a_{9}$ & $a_{10}$ & $a_{11}$ & $a_{13}$ & $a_{14}$ & $a_{15}$ & $a_{17}$ & $a_{19}$ \\
\hline$X_{0}=E$ & $9 a_{9}$ & $10 a_{10}$ & $11 a_{11}$ & $13 a_{13}$ & $14 a_{14}$ & $15 a_{15}$ & $17 a_{17}$ & $19 a_{19}$ \\
$X_{4}$ & $13 a_{13}$ & $14 a_{14}$ & $5 a_{15}$ & $-\frac{34}{3} a_{17}$ & 0 & $57 a_{19}$ & 0 & 0 \\
$X_{5}$ & $7 a_{14}$ & $10 a_{15}$ & 0 & 0 & $38 a_{19}$ & 0 & 0 & 0 \\
$X_{6}$ & $5 a_{15}$ & 0 & $17 a_{17}$ & $19 a_{19}$ & 0 & 0 & 0 & 0 \\
$X_{7}$ & 0 & 0 & 0 & 0 & 0 & 0 & 0 & 0 \\
$X_{8}$ & $-\frac{34}{3} a_{17}$ & 0 & $19 a_{19}$ & 0 & 0 & 0 & 0 & 0 \\
$X_{9}$ & 0 & $38 a_{19}$ & 0 & 0 & 0 & 0 & 0 & 0 \\
$X_{10}$ & $19 a_{19}$ & 0 & 0 & 0 & 0 & 0 & 0 & 0 \\
\hline
\end{tabular}

Devido às Proposições 1.2.14 e 2.2.3 toda restrição algébrica a $g$ representada por uma forma simplética se escreve como:

$$
a=t_{9} a_{9}+t_{10} a_{10}+t_{11} a_{11}+t_{13} a_{13}+t_{14} a_{14}+t_{15} a_{15}+t_{17} a_{17}+t_{19} a_{19}
$$

onde $t_{i} \in \mathbb{R}, i \in J=\{9,10,11,13,14,15,17,19\}$.

Suponha que $t_{i} \neq 0$, para algum $i \in J$ e $t_{u}=0$, para $u<i$. Consideremos a simetria local $\Psi_{i}:\left(\mathbb{R}^{3}, 0\right) \rightarrow\left(\mathbb{R}^{3}, 0\right)$ definida por $\Psi_{i}(x)=\left(s_{i}^{-\frac{4}{i}} x_{1}, s_{i}^{-\frac{5}{i}} x_{2}, s_{i}^{-\frac{6}{i}} x_{3}\right)$, onde $s_{i}=t_{i}$, se $i$ é ímpar e $s_{i}=\left|t_{i}\right|$ se $i$ é par. Portanto $a$ é difeomorfa a $\frac{1}{s_{i}} a$. Utilizando o Teorema 1.3 .13 e a Tabela 2.10 
concluímos que $a$ é difeomorfa a uma das restrições algébricas:

1. $a_{9}+c_{1} a_{10}+c_{2} a_{11}$

2. $\pm a_{10}+c_{1} a_{11}+c_{2} a_{13}+c_{3} a_{17}$

3. $a_{11}+c_{1} a_{13}+c_{2} a_{14}$

4. $a_{13}+c_{1} a_{14}+c_{2} a_{15}$

5. $\pm a_{14}+c_{1} a_{15}+c_{2} a_{17}$

6. $a_{15}+c a_{17}$

7. $a_{17}+c a_{19}$

8. $a_{19}$

Se $t_{i}=0$, para todo $i$, então $a=\left[\omega_{10}\right]_{g}$. No Lema 2.2.5 veremos que a órbita de $\pm a_{10}+$ $c_{1} a_{11}+c_{2} a_{13}+c_{3} a_{17}$ se decompõe nas órbitas $\pm a_{10}+c_{2} a_{13}+c_{3} a_{17} \mathrm{e} \pm a_{10}+c_{1} a_{11}+c_{2} a_{13}$.

Lema 2.2.5. Seja $a= \pm a_{10}+c_{1} a_{11}+c_{2} a_{13}+c_{3} a_{17}$. Se $c_{1} \neq 0$ então a é difeomorfa $a \pm a_{10}+c_{1} a_{11}+$ $c_{2} a_{13}$.

Demonstração. Utilizamos o método de homotopia de Moser como em [D1]. Seja $A_{t}= \pm a_{10}+$ $c_{1} a_{11}+c_{2} a_{13}+(1-t) c_{3} a_{17}$, onde $t \in[0,1]$. Suponha que exista uma família de simetrias de $g$ $\Phi_{t}:\left(\mathbb{R}^{3}, 0\right) \rightarrow\left(\mathbb{R}^{3}, 0\right), t \in[0,1]$ satisfazendo

$$
\Phi_{t}^{*} A_{t}=a, \Phi_{0}=I d .
$$

Considere $\eta$ o campo de vetores satisfazendo $\frac{d}{d t} \Phi_{t}=\eta \circ \Phi_{t}$. Segue da Proposição 1.1.21 que

$$
0=\frac{d}{d t} \Phi_{t}^{*} A_{t}=\Phi_{t}^{*}\left(\mathcal{L}_{\eta} A_{t}-c_{3} a_{17}\right)
$$

Logo

$$
\mathcal{L}_{\eta} A_{t}=c_{3} a_{17}
$$

Considere o campo de vetores $\eta=\frac{c_{3}}{17 c_{1}} X_{6}-\frac{c_{2} c_{3}}{17 c_{1}^{2}} X_{8}$, onde $X_{6}$ e $X_{9}$ são os campos de vetores descritos na Tabela 2.10. Observe que $\eta$ satisfaz (2.4). Logo a família de difeomorfismos $\Phi_{t}$ associada ao campo $\eta$ preserva $\operatorname{Im}(g)$, pois $\eta$ é levantável sobre $g$ e satisfaz (2.3).

\section{Prova do Teorema 2.2.1}


A demonstração do Teorema 2.2.1 é similar a demonstração do Teorema 2.1.1. Devido ao Teorema 1.1.18 para cada $\left[\omega_{i}\right]_{g}$ da Tabela 2.9 existe um germe de difeomorfismo $\Psi_{i}:\left(\mathbb{R}^{2 n}, 0\right) \rightarrow$ $\left(\mathbb{R}^{2 n}, 0\right)$ tal que $\Psi_{i}^{*} \omega_{i}=\omega$. Os germes de curvas da Tabela 2.8 são dados por $\Psi_{i}^{-1} \circ g$. Os invariantes simpléticos, referente a cada órbita, encontram-se nas colunas 3,4 e 5 .

Para distinguir as órbitas simplética dos germes da Tabela 2.8 usamos os invariantes simpléticos. Algumas órbitas possuem os mesmos invariantes simpléticos, porém devido à Proposição 1.4.8 e ao Corolário 1.3.6, provamos que as órbitas dos germes de curvas da Tabela 2.8 são disjuntas.

Resta verificar que os coeficientes $c, c_{1}$ e $c_{2}$ são modais. A prova em cada caso é bem similar. Para fixar ideias, provamos que $c_{1}$ e $c_{2}$ são modais na órbita de $\left[\omega_{1}\right]_{g}=a_{9}+c_{1} a_{10}+$ $c_{2} a_{11}$ da Tabela 2.8. O espaço tangente à órbita de $\left[\omega_{1}\right]_{g}$ é gerado pelas restrições algébricas $a_{19}, a_{17}, a_{15}, a_{14}, a_{13}, 9 a_{9}+10 a_{10}+11 a_{11}$. Observe que a interseção do subespaço vetorial gerado por $a_{10}$ e $a_{11}$ com o espaço tangente à órbita de $\left[\omega_{1}\right]_{g}$ é $\{0\}$. Portanto $c_{1}$ e $c_{2}$ são modais.

\subsection{Classificação simplética de curvas com semigrupo $(4,5,7)$}

Nesta seção classificamos germes de curvas parametrizadas com semigrupo $(4,5,7)$. Seguimos os passos descritos nas seção 2.1 e utilizamos o software Singular para obter alguns resultados.

Teorema 2.3.1. (i) Considere o espaço simplético $\left(\mathbb{R}^{2 n}, \omega=\sum d x_{i} \wedge d x_{n+i}\right)$. Um germe de curva $f$ : $(\mathbb{R}, 0) \rightarrow\left(\mathbb{R}^{2 n}, 0\right)$ com semigrupo $(4,5,7)$ é simplectomorfo a um, e somente um, dos germes de curvas nas linhas 1-3 da segunda coluna da Tabela 2.11 se $n=2$. Para $n>2$ é simplectomorfo a um, e somente um, dos germes de curvas da segunda coluna da Tabela 2.11.

(ii) Os parâmetros $c, c_{1}, c_{2}$ são modais. 
Tabela 2.11: Formas normais em $\mathbb{R}^{2 n}$

\begin{tabular}{clccc}
\hline & Formas normais de $f$ & $\mu_{\text {sympl }}(f)$ & $\iota(f)$ & $L t(f)$ \\
\hline 1 & $t \rightarrow\left(t^{4}, t^{5}+c t^{7}, t^{7}, 0, \ldots, 0\right)$ & 1 & 0 & 5 \\
2 & $t \rightarrow\left(t^{4}, t^{7}+c_{1} t^{9}, t^{5}, c_{2} t^{7}, 0 \ldots, 0\right)$ & 3 & 0 & 7 \\
3 & $t \rightarrow\left(t^{4}, c_{1} t^{9}, t^{5}, \pm t^{7}+c_{2} t^{9}, 0, \ldots, 0\right)$ & 4 & 0 & 7 \\
4 & $t \rightarrow\left(t^{4}, t^{9}+c_{1} t^{11}, t^{5}, c_{2} t^{9}, t^{6}, 0, \ldots, 0\right)$ & 5 & 1 & 9 \\
5 & $t \rightarrow\left(t^{4}, c_{1} t^{11}, t^{5}, \pm t^{9}, t^{7}, c_{2} t^{9}, 0, \ldots, 0\right)$ & 6 & 1 & 10 \\
6 & $t \rightarrow\left(t^{4}, t^{11}, t^{5}, c_{1} t^{12}, t^{7}, c_{2} t^{9}, 0, \ldots, 0\right)$ & 7 & 1 & 11 \\
7 & $t \rightarrow\left(t^{4}, 0, t^{5}, c_{1} t^{12}, t^{7}, \pm t^{9}+c_{2} t^{11}, 0, \ldots, 0\right)$ & 8 & 1 & 11 \\
8 & $t \rightarrow\left(t^{4}, c t^{14}, t^{5}, t^{12}, t^{7}, 0, \ldots, 0\right)$ & 8 & 1 & 13 \\
9 & $t \rightarrow\left(t^{4}, 0, t^{5}, 0, t^{7}, \pm t^{11}, 0, \ldots, 0\right)$ & 8 & 1 & 14 \\
10 & $t \rightarrow\left(t^{4}, 0, t^{5}, 0, t^{7}, 0, \ldots, 0\right)$ & 9 & $\infty$ & $\infty$ \\
\hline
\end{tabular}

Seja $g:(\mathbb{R}, 0) \rightarrow\left(\mathbb{R}^{2 n}, 0\right)$ o germe de curva parametrizada dado por $g(t)=\left(t^{4}, t^{5}, t^{7}, 0, \ldots, 0\right)$. Seja $I(g)$ o ideal dos polinômios que se anulam em $\operatorname{Im}(g)$.

Proposição 2.3.2. Um conjunto de geradores de $I(g)$ é dado por

$$
g_{12}=x_{1}^{3}-x_{2} x_{3} \quad g_{14}=x_{1} x_{2}^{2}-x_{3}^{2} \quad g_{15}=x_{1}^{2} x_{3}-x_{2}^{3}
$$

Demonstração. Um algoritmo no software Singular foi desenvolvido no Capítulo 4 para a determinação dos geradores do ideal dos polinômios que se anulam em $\operatorname{Im}(g)$.

Proposição 2.3.3. Uma base do espaço $\left[\Lambda^{2, c l}\left(\mathbb{R}^{2 n}\right)\right]_{g}$ é dada por:

$$
\begin{array}{cc}
a_{9}=\left[d x_{1} \wedge d x_{2}\right]_{g} & a_{11}=\left[d x_{1} \wedge d x_{3}\right]_{g} \\
a_{12}=\left[d x_{2} \wedge d x_{3}\right]_{g} & a_{13}=\left[x_{1} d x_{1} \wedge d x_{2}\right] \\
a_{14}=\left[x_{2} d x_{1} \wedge d x_{2}\right]_{g} & a_{15}=\left[x_{1} d x_{1} \wedge d x_{3}\right]_{g} \\
a_{16}=\left[x_{2} d x_{1} \wedge d x_{3}+x_{1} d x_{2} \wedge d x_{3}\right]_{g} & a_{17}=\left[x_{2} d x_{2} \wedge d x_{3}\right]_{g} \\
a_{18}=\left[x_{3} d x_{1} \wedge d x_{3}\right]_{g} &
\end{array}
$$

Demonstração. Assim como na Seção 2.2 utilizamos um algoritmo no software Singular para determinar uma base para $\left[\Lambda^{2, c l}\left(\mathbb{R}^{2 n}\right)\right]_{g}$. No Capítulo 4 apresentaremos o algoritmo com detalhes. 
Proposição 2.3.4. Seja a uma restrição algébrica a $g$ de uma forma simplética em $\left(\mathbb{R}^{2 n}, 0\right)$, onde $n \geq 2$. Então a é difeomorfa a uma das restrições algébricas a g nas linhas 1-3 da Tabela 2.12, para $n=2$. Se $n \geq 3$ então a é difeomorfa a uma das restrições algébricas a $g$ da Tabela 2.12.

Tabela 2.12: Restrições algébricas em $\mathbb{R}^{2 n}$

\begin{tabular}{|l|}
\hline$\left[\omega_{1}\right]_{g}=a_{9}+c a_{11}$ \\
\hline$\left[\omega_{2}\right]_{g}=a_{11}+c_{1} a_{12}+c_{2} a_{13}$ \\
\hline$\left[\omega_{3}\right]_{g}= \pm a_{12}+c_{1} a_{13}+c_{2} a_{14}$ \\
\hline$\left[\omega_{4}\right]_{g}=a_{13}+c_{1} a_{14}+c_{2} a_{15}$ \\
\hline$\left[\omega_{5}\right]_{g}= \pm a_{14}+c_{1} a_{15}+c_{2} a_{16}$ \\
\hline$\left[\omega_{6}\right]_{g}=a_{15}+c_{1} a_{16}+c_{2} a_{17}$ \\
\hline$\left[\omega_{7}\right]_{g}= \pm a_{16}+c_{1} a_{17}+c_{2} a_{18}$ \\
\hline$\left[\omega_{8}\right]_{g}=a_{17}+c a_{18}$ \\
\hline$\left[\omega_{9}\right]_{g}= \pm a_{18}$ \\
\hline$\left[\omega_{10}\right]_{g}=0$ \\
\hline \hline
\end{tabular}

Demonstração. A classificação é obtida por meio do Teorema 1.3.13. Devido às Proposições 1.2.18 e 2.3.3, é suficiente descrever os campos de vetores levantáveis sobre $g$ quase homogêneos de quase grau até 9 . Consideramos

$$
\begin{array}{ll}
X_{0}=E=4 x_{1} \frac{\partial}{\partial x_{1}}+5 x_{2} \frac{\partial}{\partial x_{2}}+7 x_{3} \frac{\partial}{\partial x_{3}} & \\
X_{3}=4 x_{3} \frac{\partial}{\partial x_{1}}+5 x_{1}^{2} \frac{\partial}{\partial x_{2}}+7 x_{2}^{2} \frac{\partial}{\partial x_{3}} & \\
X_{4}=x_{1} E & X_{5}=x_{2} E \\
X_{6}=4 x_{2}^{2} \frac{\partial}{\partial x_{1}}+5 x_{1} x_{3} \frac{\partial}{\partial x_{2}}+7 x_{1}^{2} x_{2} \frac{\partial}{\partial x_{3}} & \\
X_{7}=x_{3} E & X_{8}=x_{1}^{2} E \\
X_{9}=x_{1} x_{2} E &
\end{array}
$$

As derivadas de Lie dos elementos da base de $\left[\Lambda^{2, c l}\left(\mathbb{R}^{2 n}\right)\right]_{g}$ ao longo dos campos de vetores acima são descritos na Tabela 2.13 . 
Tabela 2.13: Derivadas de Lie

\begin{tabular}{|c|ccccccccc|}
\hline $\mathcal{L}_{X_{i}} a_{j}$ & $a_{9}$ & $a_{11}$ & $a_{12}$ & $a_{13}$ & $a_{14}$ & $a_{15}$ & $a_{16}$ & $a_{17}$ & $a_{18}$ \\
\hline$X_{0}=E$ & $9 a_{9}$ & $11 a_{11}$ & $12 a_{12}$ & $13 a_{13}$ & $14 a_{14}$ & $15 a_{15}$ & $16 a_{16}$ & $17 a_{17}$ & $18 a_{18}$ \\
$X_{3}$ & $-4 a_{12}$ & $14 a_{14}$ & $10 a_{15}$ & $-4 a_{16}$ & $-\frac{17}{3} a_{17}$ & $18 a_{18}$ & 0 & 0 & 0 \\
$X_{4}$ & $13 a_{13}$ & $15 a_{15}$ & $12 a_{16}$ & $-\frac{17}{3} a_{17}$ & $18 a_{18}$ & 0 & 0 & 0 & 0 \\
$X_{5}$ & $14 a_{14}$ & $4 a_{16}$ & $17 a_{17}$ & $18 a_{18}$ & 0 & 0 & 0 & 0 & 0 \\
$X_{6}$ & $5 a_{15}$ & $\frac{17}{3} a_{17}$ & $-9 a_{18}$ & 0 & 0 & 0 & 0 & 0 & 0 \\
$X_{7}$ & $-4 a_{16}$ & $18 a_{18}$ & 0 & 0 & 0 & 0 & 0 & 0 & 0 \\
$X_{8}$ & $-\frac{17}{3} a_{17}$ & 0 & 0 & 0 & 0 & 0 & 0 & 0 & 0 \\
$X_{9}$ & $18 a_{18}$ & 0 & 0 & 0 & 0 & 0 & 0 & 0 & 0 \\
\hline
\end{tabular}

Seja $a$ uma restrição algébrica a $g$ de uma forma simplética em $\mathbb{R}^{2 n}, n \geq 2$. Devido às Proposições 1.2.14 e 2.3.3, $a$ se escreve como

$$
a=t_{9} a_{9}+t_{11} a_{11}+t_{12} a_{12}+t_{13} a_{13}+t_{14} a_{14}+t_{15} a_{15}+t_{16} a_{16}+t_{17} a_{17}+t_{18} a_{18} .
$$

$t_{i} \in \mathbb{R}$, para todo $i \in\{9,11,12,13,14,15,16,17,18\}$.

Suponha que $t_{i} \neq 0$, para algum $i$. Neste caso, consideremos a simetria local $\Psi_{i}:\left(\mathbb{R}^{3}, 0\right) \rightarrow$ $\left(\mathbb{R}^{3}, 0\right)$ definida por $\Psi_{i}(x)=\left(s_{i}^{-\frac{4}{i}} x_{1}, s_{i}^{-\frac{5}{i}} x_{2}, s_{i}^{-\frac{7}{i}} x_{3}\right)$, onde $s_{i}=t_{i}$ se $i$ é ímpar, e $s_{i}=\left|t_{i}\right|$ se $i$ é par. Portanto $a$ é difeomorfa a $\frac{1}{s_{i}} a$. Aplicando o Teorema 1.3.13 concluímos que $a$ é difeomorfa a uma das restrições algébricas das linhas 1-9 da Tabela 2.12. Se $t_{i}=0$, para todo $i$, então $a=\left[\omega_{10}\right]_{g}$.

\section{Prova do Teorema 2.3.1}

A prova deste resultado é similar a do Teorema 2.1.1. Devido ao Teorema de 1.1.18, para cada $\left[\omega_{i}\right]_{g}$ da Tabela 2.12 existe um germe de difeomorfismo $\Psi_{i}:\left(\mathbb{R}^{2 n}, 0\right) \rightarrow\left(\mathbb{R}^{2 n}, 0\right)$ tal que $\Psi_{i}^{*} \omega_{i}=\omega$. Os germes de curvas da Tabela 2.11 são dados por $\Psi_{i}^{-1} \circ g$. Os invariantes simpléticos, referentes a cada curva, encontram-se nas colunas 3,4 e 5 da Tabela 2.11.

Para distinguir as órbitas utilizamos os invariantes simpléticos descritos na Tabela 2.11. Por fim, verificamos que os coeficientes $c, c_{1}$ e $c_{2}$ são modais. A prova em cada caso é bem similar. Para fixar ideias, provamos que $c_{1}$ e $c_{2}$ são modais na órbita de $\left[\omega_{2}\right]_{g}=a_{11}+c_{1} a_{12}+$ $c_{2} a_{13}$ da Tabela 2.11. O espaço tangente à órbita de $\left[\omega_{2}\right]_{g}$ é gerado pelas restrições algébricas $a_{18}, a_{17}, a_{16}, a_{15}, a_{14}, 11 a_{11}+12 a_{12}+13 a_{13}$. Observe que a interseção do subespaço vetorial gerado por $a_{12}$ e $a_{13}$ com o espaço tangente à órbita de $\left[\omega_{2}\right]_{g}$ é $\{0\}$. Portanto $c_{1}$ e $c_{2}$ são modais. 


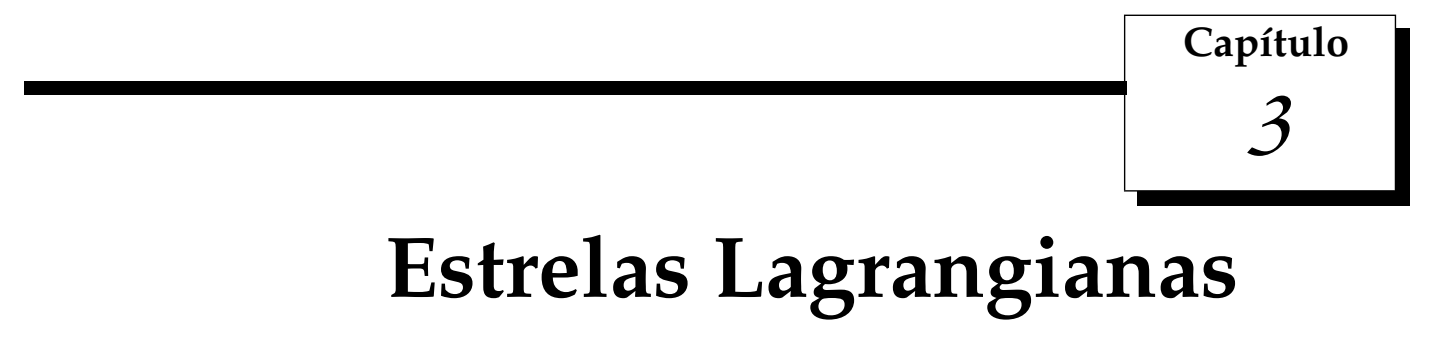

\subsection{Introdução}

O problema de classificação de estrelas lagrangianas foi inicialmente estudado por S. Janeczko em [J]. Neste capítulo apresentaremos a classificação das estrelas lagrangianas quase homogêneas transversais em $\left(\left(\mathbb{R}^{2 n}, \omega\right), 0\right)$ sob a ação do grupo dos simplectomorfismos utilizando o método das restrições algébricas.

Neste capítulo os germes considerados são germes na origem na categoria real analítica. Além disso, consideramos $\left(\mathbb{R}^{2 n}, \omega=\sum_{i=1}^{n} d x_{i} \wedge d y_{i}\right)$ o espaço euclidiano simplético $2 n$ dimensional com o sistema de coordenadas $\left(x_{1}, \ldots, x_{n}, y_{1}, \ldots, y_{n}\right)$.

Sejam $L_{1}, \ldots, L_{s}$ germes de subvariedades lagrangianas de $\left(\left(\mathbb{R}^{2 n}, \omega\right), 0\right)$ se intersectando na origem.

Definição 3.1.1. O conjunto $\left(\left\{L_{1}, \ldots, L_{s}\right\}, 0\right)$ é chamado s-estrela lagrangiana em $\left(\left(\mathbb{R}^{2 n}, \omega\right), 0\right)$. Se $s=2$ e $L_{1}$ é transversal a $L_{2}$ então a 2-estrela lagrangiana $\left(\left\{L_{1}, L_{2}\right\}, 0\right)$ é chamada estrela lagrangiana básica. Uma 3-estrela lagrangiana é chamada, por simplicidade, de estrela lagrangiana.

Seja $E=\left(\left\{L_{1}, \ldots, L_{s}\right\}, 0\right)$ uma $s$-estrela lagrangiana em $\left(\left(\mathbb{R}^{2 n}, \omega\right), 0\right)$. Diremos que $E$ é quase homogênea se $L=L_{1} \cup \cdots \cup L_{s}$ é um germe quase homogêneo. Diremos também que $E$ é transversal se $L_{1}, \ldots, L_{s}$ são germes dois a dois transversais se intersectando apenas na origem.

Sejam $E=\left(\left\{L_{1}, \ldots, L_{s}\right\}, 0\right)$ e $E^{\prime}=\left(\left\{L_{1}^{\prime}, \ldots, L_{s}^{\prime}\right\}, 0\right)$ duas $s$-estrelas lagrangianas. Diremos que são difeomorfas se existem $j_{1}, \ldots, j_{s}$ uma permutação de $1, \ldots, s$ e um germe de difeomorfismo $\Phi:\left(\left(\mathbb{R}^{2 n}, \omega\right), 0\right) \rightarrow\left(\left(\mathbb{R}^{2 n}, \omega\right), 0\right)$ tal que $\Phi\left(L_{i}\right)=L_{j_{i}}^{\prime}$ para todo $i \in\{1, \ldots, s\}$. Quando $\Phi$ for um germe de simplectomorfismo de $\left(\left(\mathbb{R}^{2 n}, \omega\right), 0\right)$ diremos que $E$ e $E^{\prime}$ são simpleticamente equivalentes, ou simplesmente, equivalentes.

Dada uma estrela lagrangiana básica $\left(\left\{L_{1}, L_{2}\right\}, 0\right)$ em $\left(\left(\mathbb{R}^{2 n}, \omega\right), 0\right)$, existe um simplectomorfismo $\Phi:\left(\left(\mathbb{R}^{2 n}, \omega\right), 0\right) \rightarrow\left(\left(\mathbb{R}^{2 n}, \omega\right), 0\right)$ que leva $L_{1}$ em $\left\{y_{1}=\cdots=y_{n}=0\right\}$ e $L_{2}$ em $\left\{x_{1}=\cdots=x_{n}=0\right\}$ (veja [AVG]). 
Seja $\left(\left\{L_{1}, \ldots, L_{s}\right\}, 0\right)$ um $s$-estrela lagrangiana. Denotaremos a parte regular de $L$ como $L_{\text {reg. }}$

Notações: Seja $\theta$ uma $k$-forma e $E=\left(\left\{L_{1}, \ldots, L_{s}\right\}, 0\right)$ uma $s$-estrela lagrangiana ambas em $\left(\left(\mathbb{R}^{2 n}, \omega\right), 0\right)$.

1. A restrição de $\theta$ ao conjunto $\left\{\left(p, v_{1}, \ldots, v_{k}\right) \mid p \in L_{j}\right.$ e $\left.v_{1}, \ldots, v_{k} \in T_{p} L_{j}\right\}$ será denotada por $\left.\theta\right|_{T L_{j}}, j=1, \ldots, s$.

2. Suponhamos que para cada $p \in L_{\text {reg }}$ e $u_{1}, \ldots, u_{k} \in T_{p} L_{\text {reg }}$ tem-se $\theta(p)\left(u_{1}, \ldots, u_{k}\right)=0$. Nesse caso, diremos que $\theta$ se anula em $T L_{\text {reg }}$.

Observe que se $E$ for transversal então a única singularidade de $L$ é a origem. Portanto, se $\sigma$ é uma forma simplética que se anula em $T L_{\text {reg }}$ então, por continuidade, $L_{j}$ é um germe de subvariedade lagrangiana em $\left(\left(\mathbb{R}^{2 n}, \sigma\right), 0\right), j=1, \ldots, s$.

Lema 3.1.2. Sejam $\Phi:\left(\mathbb{R}^{2 n}, 0\right) \rightarrow\left(\mathbb{R}^{2 n}, 0\right)$ um germe de difeomorfismo e $N$ um germe de subvariedade de $\left(\mathbb{R}^{2 n}, 0\right)$. Então $W=\Phi(N)$ é um germe de subvariedade lagrangiana com respeito ao espaço simplético $\left(\left(\mathbb{R}^{2 n}, \omega\right), 0\right)$ se, e somente se, $N$ é um germe de subvariedade lagrangiana com respeito ao espaço simplético $\left(\left(\mathbb{R}^{2 n}, \Phi^{*} \omega\right), 0\right)$.

Demonstração. Suponhamos que $W$ seja um germe de subvariedade lagrangiana em $\left(\left(\mathbb{R}^{2 n}, \omega\right), 0\right)$. Considere $p \in N$ e $u_{1}, u_{2} \in T_{p} N$. Então

$$
0=\omega(\Phi(p))\left(d \Phi(p) \cdot u_{1}, d \Phi(p) \cdot u_{2}\right)=\Phi^{*} \omega(p)\left(u_{1}, u_{2}\right)
$$

Portanto $\left.\Phi^{*} \omega\right|_{T N}=0$.

A recíproca é análoga.

Corolário 3.1.3. (Corolário Teorema A) Sejam $\tilde{E}=\left(\left\{\tilde{L_{1}}, \ldots, \tilde{L_{s}}\right\}, 0\right)$ e $\hat{E}=\left(\left\{\hat{L_{1}}, \ldots, \hat{L_{s}}\right\}, 0\right) s$ estrelas lagrangianas difeomorfas a uma s-estrela lagrangiana quase homogênea $E=\left(\left\{L_{1}, \ldots, L_{s}\right\}, 0\right)$. Então $\tilde{E}$ e $\hat{E}$ são equivalentes se, e somente se, $[\omega]_{\hat{L}}$ e $[\omega]_{\tilde{L}}$ são difeomorfas.

Demonstração. Suponha que $\tilde{E}$ e $\hat{E}$ sejam equivalentes. Considere o simplectomorfismo $\Psi$ : $\left(\left(\mathbb{R}^{2 n}, \omega\right), 0\right) \rightarrow\left(\left(\mathbb{R}^{2 n}, \omega\right), 0\right)$ tal que $\Psi\left(\hat{L}_{i}\right)=\tilde{L}_{j_{i}}, i \in\{1, \ldots, s\}$, e $j_{1}, \ldots, j_{s}$ uma permutação de $1, \ldots, s . \operatorname{Logo}$

$$
\Psi^{*}[\omega]_{\tilde{L}}=[\omega]_{\hat{L}}
$$

Reciprocamente, suponhamos que $[\omega]_{\hat{L}}$ e $[\omega]_{\tilde{L}}$ são difeomorfas. Considere os germes de difeomorfismos $\phi_{1}$ e $\phi_{2}$ de $\left(\mathbb{R}^{2 n}, 0\right)$ tais que

$$
\phi_{1}(\hat{L})=L \text { e } \phi_{2}(\tilde{L})=L
$$


Sejam $\sigma_{1}=\left(\phi_{1}^{-1}\right)^{*} \omega$ e $\sigma_{2}=\left(\phi_{2}^{-1}\right)^{*} \omega$. Logo $\left[\sigma_{1}\right]_{L}$ e $\left[\sigma_{2}\right]_{L}$ são difeomorfas a $[\omega]_{\hat{L}}$ e $[\omega]_{\tilde{L}^{\prime}}$, respectivamente. Seja $\rho:\left(\mathbb{R}^{2 n}, 0\right) \rightarrow\left(\mathbb{R}^{2 n}, 0\right)$ a simetria local de $L$ tal que

$$
\rho^{*}\left[\sigma_{1}\right]_{L}=\left[\rho^{*} \sigma_{1}\right]_{L}=\left[\sigma_{2}\right]_{L}
$$

De acordo com o Lema 3.1.2, $E$ é uma $s$-estrela lagrangiana com respeito a $\left(\left(\mathbb{R}^{2 n}, \sigma_{1}\right), 0\right)$ e $\left(\left(\mathbb{R}^{2 n}, \sigma_{2}\right), 0\right)$. Segue do Teorema A que existe um germe de difeomorfismo $H:\left(\mathbb{R}^{2 n}, 0\right) \rightarrow$ $\left(\mathbb{R}^{2 n}, 0\right)$ tal que $\left.H\right|_{L}=I d$ e $H^{*} \sigma_{2}=\rho^{*} \sigma_{1}$. Considere $T=\phi_{2}^{-1} \circ H \circ \rho^{-1} \circ \phi_{1}$. Logo

$$
T^{*} \omega=\omega \text { e } T(\hat{L})=\tilde{L}
$$

Segue que $\hat{E}$ e $\tilde{E}$ são equivalentes.

Observação 3.1.4. Seja $E=\left(\left\{L_{1}, \ldots, L_{s}\right\}, 0\right)$ uma s-estrela lagrangiana transversal quase homogênea. De acordo com a demonstração do Corolário 3.1.3, a classificação de s-estrelas lagrangianas transversais se reduz à classificação de restrições algébricas a $L$ de formas simpléticas que se anulam em $T L_{\text {reg. }}$.

Proposição 3.1.5. Seja $\tilde{E}=\left(\left\{\tilde{L}_{1}, \tilde{L}_{2}, \tilde{L}_{3}\right\}, 0\right)$ uma estrela lagrangiana transversal em $\left(\left(\mathbb{R}^{2 n}, \omega\right), 0\right)$. Existe uma mudança de coordenadas em $\left(\mathbb{R}^{2 n}, 0\right)$ tal que, para cada $i, \tilde{L}_{i}$ é difeomorfa a $L_{i}$, onde $L_{1}=$ $\left\{y_{1}=\cdots=y_{n}=0\right\}, L_{2}=\left\{x_{1}=\cdots=x_{n}=0\right\}$ e $L_{3}=\left\{x_{1}-y_{1}=\cdots=x_{n}-y_{n}=0\right\}$.

Demonstração. Sendo $\left(\left(\mathbb{R}^{2 n}, \omega\right), 0\right)$ um espaço simplético e $\tilde{L}_{1}$ transversal a $\tilde{L}_{2}$ então existe um simplectomorfismo, em particular um difeomorfismo, $\Phi:\left(\left(\mathbb{R}^{2 n}, \omega\right), 0\right) \rightarrow\left(\left(\mathbb{R}^{2 n}, \omega\right), 0\right)$ tal que $\Phi\left(\tilde{L_{1}}\right)=L_{1}$ e $\Phi\left(\tilde{L_{2}}\right)=L_{2}$ (veja $[\mathrm{AVG}]$ ).

Seja $\hat{L_{3}}=\Phi\left(\tilde{L_{3}}\right)$. Como difeomorfismo preserva transversalidade, então $\hat{L_{3}}$ é transversal a $L_{1}$ e a $L_{2}$. Como $\Phi$ é um simplectomorfismo segue que $\hat{L_{3}}$ é lagrangiana em $\left(\left(\mathbb{R}^{2 n}, \omega\right), 0\right)$. Devido ao Exemplo 1.1.16, $\hat{L_{3}}$ se escreve como

$$
y_{i}=\frac{\partial H}{\partial x_{i}}(x), i=1, \ldots, n
$$

para algum germe de função suave $H:\left(\mathbb{R}^{n}, 0\right) \rightarrow \mathbb{R}$. Como $\hat{L_{3}}$ é transversal a $L_{2}$, segue que o germe $G:\left(\mathbb{R}^{n}, 0\right) \rightarrow\left(\mathbb{R}^{n}, 0\right)$ definido por $G(x)=\left(\frac{\partial H}{\partial x_{1}}(x), \ldots, \frac{\partial H}{\partial x_{n}}(x)\right)$ é um difeomorfismo. Considere $\Psi:\left(\mathbb{R}^{2 n}, 0\right) \rightarrow\left(\mathbb{R}^{2 n}, 0\right)$ definido por $\Psi(x, y)=\left(x, G^{-1}(y)\right)$. Assim $\Psi\left(L_{i}\right)=L_{i}$, para $i=1,2$, e $\Psi\left(\hat{L_{3}}\right)=L_{3}=\left\{x_{1}-y_{1}=\cdots=x_{n}-y_{n}=0\right\}$.

No que segue, $E=\left(\left\{L_{1}, L_{2}, L_{3}\right\}, 0\right)$ é a estrela lagrangiana em $\left(\left(\mathbb{R}^{2 n}, \omega\right), 0\right)$ onde $L_{1}=\left\{y_{1}=\cdots=y_{n}=0\right\}, L_{2}=\left\{x_{1}=\cdots=x_{n}=0\right\}$ e $L_{3}=\left\{x_{1}-y_{1}=\cdots=x_{n}-y_{n}=0\right\}$.

Seja $k$ um inteiro não negativo. Fixaremos algumas notações: 
- $\Lambda_{\text {reg }}^{k}\left(\mathbb{R}^{2 n}\right)$ : o espaço vetorial das $k$-formas que se anulam em $T L_{\text {reg. }}$.

- $\left[\Lambda_{\text {reg }}^{k}\left(\mathbb{R}^{2 n}\right)\right]_{L}$ : o espaço vetorial das restrições algébricas a $L$ de elementos de $\Lambda_{\text {reg }}^{k}\left(\mathbb{R}^{2 n}\right)$.

- $\Lambda_{\text {reg }}^{k, c l}\left(\mathbb{R}^{2 n}\right)$ : o subespaço vetorial de $\Lambda_{\text {reg }}^{k}\left(\mathbb{R}^{2 n}\right)$ das $k$-formas fechadas que se anulam em $T L_{\mathrm{reg}}$.

- $\left[\Lambda_{\text {reg }}^{k, c l}\left(\mathbb{R}^{2 n}\right)\right]_{L}$ : o subespaço de $\left[\Lambda_{\text {reg }}^{k}\left(\mathbb{R}^{2 n}\right)\right]_{L}$ das restrições algébricas a $L$ de elementos de $\Lambda_{\text {reg }}^{k, \mathrm{cl}}\left(\mathbb{R}^{2 n}\right)$.

- $S y m p_{\text {reg }}\left(\mathbb{R}^{2 n}\right)$ : o subconjunto aberto de $\Lambda_{\text {reg }}^{2, c l}\left(\mathbb{R}^{2 n}\right)$ das formas simpléticas que se anulam em $T L_{\text {reg }}$

- $\left[\operatorname{Symp}_{\text {reg }}\left(\mathbb{R}^{2 n}\right)\right]_{L}$ : o subconjunto de $\left[\Lambda_{\text {reg }}^{2, c l}\left(\mathbb{R}^{2 n}\right)\right]_{L}$ das restrições algébricas a $L$ de elementos de $\operatorname{Symp}_{\mathrm{reg}}\left(\mathbb{R}^{2 n}\right)$.

Este capítulo está organizado da seguinte maneira: na seção 3.2 encontramos um conjunto finito de geradores de $\left[\Lambda_{\text {reg }}^{2, c l}\left(\mathbb{R}^{2 n}\right)\right]_{L}$. Provamos na seção 3.3 que é possível reduzir a classificação das restrições algébricas a $L$ em $\left[\operatorname{Symp}_{\text {reg }}\left(\mathbb{R}^{2 n}\right)\right]_{L}$ sob a ação das simetrias locais de $L$ à classificação de restrições algébricas a $L$ de formas simpléticas homogêneas de grau 2 se anulando em $T L_{\text {reg }}$ sob a ação das simetrias locais de $L$ lineares. Na seção 3.4 classificamos as estrelas lagrangianas transversais em $\left(\left(\mathbb{R}^{2 n}, \omega\right), 0\right)$ utilizando o método das restrições algébricas. Finalizamos o capítulo na seção 3.5 com o índice de Maslov.

\subsection{Geradores de $\left[\Lambda_{\text {reg }}^{2, c l}\left(\mathbb{R}^{2 n}\right)\right]_{L}$}

Nesta seção encontraremos um conjunto finito de geradores para o espaço vetorial $\left[\Lambda_{\text {reg }}^{2, \mathrm{cl}}\left(\mathbb{R}^{2 n}\right)\right]_{L}$. Um resultado útil é o seguinte.

Proposição 3.2.1. Seja $\theta$ uma $k$-forma que se anula em $T L_{\mathrm{reg}}$. Então $\theta^{(r)}$ se anula $e m T L_{\mathrm{reg}}$, para todo inteiro $r \geq k$.

Demonstração. Sendo $\theta$ uma $k$-forma então $\theta^{(r)}=0$ para todo $r=0, \ldots, k-1$. Seja $r \geq k$ então $\theta$ se decompõe como

$$
\theta=\theta^{(k)}+\cdots+\theta^{(r)}+T
$$

onde $\theta^{(s)}$ é uma $k$-forma homogênea de grau $s, s=k, \ldots, r$, e $T$ é uma $k$-forma $\operatorname{com} T^{(i)}=0$, $i=0, \ldots, r$.

Sejam $p \in L_{j}$ e $u_{1}, \ldots, u_{k} \in T_{p} L_{j}=L_{j}$, para algum $j \in\{1,2,3\}$. Escrevendo $u=$ $\left(u_{1}, \ldots, u_{k}\right)$ então para $t \neq 0$ suficientemente pequeno tem-se

$$
0=\theta(t p) u=\theta^{(k)}(p) u+\cdots+t^{r-k} \theta^{(r)}(p) u+T(t p) u .
$$


Pela fórmula da expansão de Taylor tem-se $\lim _{t \rightarrow 0} \frac{T(t p)}{t^{r-k}}=0$. Portanto

$$
0=\lim _{t \rightarrow 0} \theta(t p) u=\theta^{(k)}(p) u
$$

Consequentemente,

$$
0=\lim _{t \rightarrow 0} \frac{\theta(t p) u}{t}=\theta^{(k+1)}(p) u
$$

Logo $\theta^{(k)}$ e $\theta^{(k+1)}$ se anulam em $T L_{j}$. Seguindo o mesmo procedimento concluímos que $\theta^{(r)}$ se anula em $T L_{j}$, para todo $j \in\{1,2,3\}$. Portanto $\theta^{(r)}$ se anula em $T L_{\text {reg. }}$.

No que segue, mostraremos que os geradores de $\left[\Lambda_{\text {reg }}^{2, \mathrm{cl}}\left(\mathbb{R}^{2 n}\right)\right]_{L}$ são obtidos como diferenciais de geradores de $\left[\Lambda_{\text {reg }}^{1}\left(\mathbb{R}^{2 n}\right)\right]_{L}$. Reduzimos assim nosso problema ao caso de 1 -formas.

Proposição 3.2.2. Seja $\sigma$ uma 2-forma analítica em $\Lambda_{\text {reg }}^{2, c l}\left(\mathbb{R}^{2 n}\right)$. Então existe uma 1-forma analítica $\gamma$ em $\Lambda_{\text {reg }}^{1}\left(\mathbb{R}^{2 n}\right)$ tal que $\sigma=d \gamma$.

Demonstração. Definimos $F:[0,1] \times \mathbb{R}^{2 n} \rightarrow \mathbb{R}^{2 n}$ por $F(t, x, y)=F_{t}(x, y)=e^{-t}(x, y)$. É fácil verificar que $X=\sum_{i=1}^{n}-\left(\frac{\partial}{\partial x_{i}}+\frac{\partial}{\partial y_{i}}\right)$ é o campo de vetores associado à equação $\frac{d F_{t}}{d t}=X \circ F_{t}$. Segue da Proposição 3.2.1 que $\sigma^{(r)} \in \Lambda_{\text {reg }}^{2, c l}\left(\mathbb{R}^{2 n}\right)$, para todo $r \geq 2$. Temos que

$$
\begin{aligned}
\left(e^{-r}-1\right) \sigma^{(r)} & =F_{1}{ }^{*} \sigma^{(r)}-F_{0}{ }^{*} \sigma^{(r)}=\int_{0}^{1} \frac{d}{d t} F_{t}^{*} \sigma^{(r)} d t=\int_{0}^{1} F_{t}^{*}\left(\mathcal{L}_{X} \sigma^{(r)}\right) d t \\
& =\int_{0}^{1} F_{t}^{*}\left(d\left(i_{X} \sigma^{(r)}\right)\right) d t=d \int_{0}^{1} F_{t}^{*}\left(i_{X} \sigma^{(r)}\right) d t .
\end{aligned}
$$

Sejam $p \in L_{\mathrm{reg}}, v \in T_{p} L_{\mathrm{reg}}$ e $t \in[0,1]$. Então

$$
F_{t}^{*}\left(i_{X} \sigma^{(r)}\right)(p) \cdot v=\sigma^{(r)}\left(F_{t}(p)\right)\left(X \circ F_{t}(p), d F_{t}(p) \cdot v\right)=0
$$

pois $L_{\text {reg }}=L_{1} \cup L_{2} \cup L_{3}-\{0\}$ e $F_{t}(L-\{0\}) \subset L-\{0\}, t \in[0,1]$. Portanto $\int_{0}^{1} F_{t}^{*}\left(i_{X} \sigma^{(r)}\right) d t \in$ $\Lambda_{\text {reg }}^{1}\left(\mathbb{R}^{2 n}\right)$ e, consequentemente,

$$
\gamma_{r}=\frac{1}{\left(e^{-r}-1\right)} \int_{0}^{1} F_{t}^{*}\left(i_{X} \sigma^{(r)}\right) d t \in \Lambda_{\mathrm{reg}}^{1}\left(\mathbb{R}^{2 n}\right)
$$

Portanto $\sigma=\sum_{r} \sigma^{(r)}=d\left(\sum_{r} \gamma_{r}\right)$.

Proposição 3.2.3. Seja $\gamma \in \Lambda_{\text {reg }}^{1}\left(\mathbb{R}^{2 n}\right)$ então $d \gamma \in \Lambda_{\text {reg }}^{2, c l}\left(\mathbb{R}^{2 n}\right)$. 
Demonstração. Temos que $\gamma$ se escreve como

$$
\gamma=\sum_{j=1}^{n}\left(f_{j} d x_{j}+g_{j} d y_{j}\right)
$$

onde $f_{j}$ e $g_{j}$ são germes de funções em $\left(\mathbb{R}^{2 n}, 0\right), j=1, \ldots, n$. Temos que

$$
\begin{aligned}
& d \gamma=\sum_{j=1}^{n}\left(d f_{j} \wedge d x_{j}+d g_{j} \wedge d y_{j}\right)= \\
& \sum_{i, j=1}^{n}\left(\left(\frac{\partial f_{j}}{\partial x_{i}} d x_{i}+\frac{\partial f_{j}}{\partial y_{i}} d y_{i}\right) \wedge d x_{j}+\left(\frac{\partial g_{j}}{\partial x_{i}} d x_{i}+\frac{\partial g_{j}}{\partial y_{i}} d y_{i}\right) \wedge d y_{j}\right) .
\end{aligned}
$$

Como $\left.\gamma\right|_{T L_{\text {reg }}}=0$ então $f_{j}(x, 0)=0, g_{j}(0, y)=0$ e $\left(f_{j}+g_{j}\right)(z, z)=0$. Segue que

$$
\left.d \gamma\right|_{T L_{1}}=\sum_{i, j=1}^{n} \frac{\partial f_{j}}{\partial x_{i}}(x, 0) d x_{i} \wedge d x_{j}=\sum_{j=1}^{n} d\left(f_{j}(x, 0)\right) \wedge d x_{j}=0
$$

Analogamente $\left.d \gamma\right|_{T L_{2}}=0$. Também tem-se que

$$
\begin{aligned}
\left.d \gamma\right|_{T L_{3}} & =\sum_{i, j=1}^{n}\left(\left(\frac{\partial f_{j}}{\partial z_{i}}(z, z)+\frac{\partial g_{j}}{\partial z_{i}}(z, z)\right) d z_{i} \wedge d z_{j}\right) \\
& =\sum_{j=1}^{n}\left(d\left(f_{j}(z, z)+g_{j}(z, z)\right) \wedge d z_{j}\right)=0 .
\end{aligned}
$$

A seguir descrevemos $\mathscr{A}_{0}^{k}\left(L,\left(\mathbb{R}^{2 n}, 0\right)\right)$. Observe que se $\theta$ é uma $k$-forma em $\mathscr{A}_{0}^{k}\left(L,\left(\mathbb{R}^{2 n}, 0\right)\right)$ então $\theta^{(r)} \in \mathscr{A}_{0}^{k}\left(L,\left(\mathbb{R}^{2 n}, 0\right)\right)$, para todo inteiro não negativo $r$. Portanto, $\mathscr{A}_{0}^{k}\left(L,\left(\mathbb{R}^{2 n}, 0\right)\right)$ é gerado por $k$-formas homogêneas.

Seja $\alpha+d \beta \in \mathscr{A}_{0}^{k}\left(L,\left(\mathbb{R}^{2 n}, 0\right)\right)$, onde $\alpha$ é uma $k$-forma e $\beta$ é uma $(k-1)$-forma, ambas homogêneas de grau $r$ e se anulando em $L$. As formas $\alpha$ e $\beta$ se escrevem como

$$
\begin{aligned}
& \alpha=\sum \alpha_{i_{1} \cdots i_{k}}(x, y) d x_{i_{1}} \wedge \ldots \wedge d x_{i_{u}} \wedge d y_{i_{u+1}} \wedge \ldots \wedge d y_{i_{k}} \mathrm{e} \\
& \beta=\sum \beta_{j_{1} \cdots j_{k-1}}(x, y) d x_{j_{1}} \wedge \ldots \wedge d x_{j_{v}} \wedge d y_{j_{v+1}} \wedge \ldots \wedge d y_{j_{k-1}},
\end{aligned}
$$


onde $\alpha_{i_{1} \cdots i_{k}}$ e $\beta_{j_{1} \cdots j_{k-1}}$ são polinômios homogêneos de graus $r-k$ e $r-k+1$, respectivamente. Como $\alpha$ e $\beta$ se anulam em $L$, então, por definição, $\alpha_{i_{1} \cdots i_{k}}$ e $\beta_{j_{1} \cdots j_{k-1}}$ se anulam em $L$. Como $\alpha_{i_{1} \cdots i_{k}}$ e $\beta_{j_{1} \cdots+j_{k-1}}$ são polinômios, segue que $\alpha_{i_{1} \cdots i_{k}}, \beta_{i_{1} \cdots i_{k-1}} \in I(L)$, onde $I(L)$ é o ideal dos polinômios que se anulam em $L$. A seguir encontramos um conjunto finito de geradores para $I(L)$.

Proposição 3.2.4. $I(L)$ é gerado pelos polinômios:

de grau 2: $x_{i} y_{j}-x_{j} y_{i}, 1 \leq i<j \leq n$.

de grau 3: $x_{i} x_{j} y_{k}-y_{i} y_{j} x_{k}, 1 \leq i \leq j \leq k \leq n$.

Demonstração. É fácil verificar que se um polinômio pertence a $I(L)$ então cada parte homogênea também pertence a $I(L)$. Portanto $I(L)$ é gerado por polinômios homogêneos que se anulam em $L$. Obviamente não há polinômios de grau 1 que se anulam em $L$.

Seja $\tilde{f}$ um polinômio em $\mathbb{R}^{2 n}$ que se anula em $L$. Como $\tilde{f}$ se anula em $L_{1} \cup L_{2}$, segue do Lema 1.2.19 que $\tilde{f}$ é da forma

$$
\tilde{f}(x, y)=\sum_{i, j=1}^{n} x_{i} y_{j} \tilde{f_{i j}}(x, y) .
$$

onde $\tilde{f_{i j}}$ é um polinômio em $\mathbb{R}^{2 n}$.

Seja $f \in I(L)$ um polinômio homogêneo de grau 2. Segue da observação acima que $f$ é da forma $f=\sum_{i, j=1}^{n} a_{i j} x_{i} y_{j}$, onde $a_{i j} \in \mathbb{R}, i, j=1, \ldots, n$. Como $\left.f\right|_{L_{3}}=0$ então $a_{i j}=-a_{j i}$, para todo $i, j \in\{1, \ldots, n\}$. Portanto os geradores de grau 2 são:

- $x_{i} y_{j}-x_{j} y_{i}, 1 \leq i<j \leq n$.

Analogamente os polinômios homogêneos geradores de grau 3 são:

- $x_{i} x_{j} y_{k}-x_{i} y_{j} x_{k}$

- $x_{i} x_{j} y_{k}-y_{i} x_{j} x_{k}$

- $x_{i} x_{j} y_{k}-x_{i} y_{j} y_{k}$

- $x_{i} x_{j} y_{k}-y_{i} x_{j} y_{k}$

- $x_{i} x_{j} y_{k}-y_{i} y_{j} x_{k}$

para $i, j, k \in\{1, \ldots, n\}$. É fácil verificar que os polinômios homogêneos de grau 3 em $I(L)$ são gerados por polinômios de grau 2 em $I(L)$ e polinômios da forma $x_{i} x_{j} y_{k}-y_{j} y_{i} x_{k}$, para 
$1 \leq i \leq j \leq k \leq n$. Portanto os polinômios em $I(L)$ de grau até 3 são gerados pelo conjunto $S=\left\{x_{i} y_{j}-x_{j} y_{i} ; 1 \leq i<j \leq n\right\} \cup\left\{x_{i} x_{j} y_{k}-y_{i} y_{j} x_{k} ; 1 \leq i \leq j \leq k \leq n\right\}$. Seja $I_{S}$ o ideal gerado por $S$. Usamos o princípio da indução para provar que $I_{S}=I(L)$.

Suponhamos que os polinômios homogêneos de grau $l-1 \geq 3$ em $I(L)$ pertençam a $I_{S}$. A seguir, provamos que os polinômios homogêneos de grau $l$ em $I(L)$ pertençam a $I_{S}$.

Seja $g(x, y) \in I(L)$ um polinômio homogêneo de grau $l$. Então $g$ é da forma:

$$
\begin{aligned}
& g(x, y)=\sum_{i_{1}, \ldots, i_{l}=1}^{n} a_{i_{1} \cdots i_{l}}^{(1)} y_{i_{1}} x_{i_{2}} \ldots x_{i_{l}}+\cdots+ \\
& \sum_{i_{1}, \ldots, i_{l}=1}^{n} a_{i_{1} \cdots i_{l}}^{(t)} y_{i_{1}} \cdots y_{i_{t}} x_{i_{t+1}} \ldots x_{i_{l}}+\cdots+\sum_{i_{1}, \ldots, i_{l}=1}^{n} a_{i_{1} \cdots i_{l}}^{(l-1)} y_{i_{1}} \ldots y_{i_{l-1}} x_{i_{l}} .
\end{aligned}
$$

Como $g$ se anula em $L_{3}$ tem-se que

$$
\sum_{\sigma \in S_{l}} a_{\sigma\left(i_{1}\right) \cdots \sigma\left(i_{l}\right)}^{(1)}+\cdots+\sum_{\sigma \in S_{l}} a_{\sigma\left(i_{1}\right) \cdots \sigma\left(i_{l}\right)}^{(l-1)}=0
$$

onde $S_{l}$ é o grupo de permutações de $\left\{i_{1}, \ldots, i_{l}\right\}$. Portanto os polinômios em $I(L)$ homogêneos de grau $l$ são gerados pelos polinômios da forma

$$
h_{i j t}=y_{i_{1}} x_{i_{2}} \cdots x_{i_{l}}-y_{j_{1}} \cdots y_{j_{t}} x_{j_{t+1}} \cdots x_{j_{l}}
$$

para $1 \leq t \leq l-1$ e $\left\{j_{1}, \ldots, j_{l}\right\}=\left\{i_{1} \ldots, i_{l}\right\}$. Como $x_{i} y_{j}-y_{i} x_{j} \in I_{S}, i, j=1, \ldots, n$, concluímos que se $t=1$ então $h_{i j 1} \in I(L)$ e se $t>1$ então

$$
h_{i j t} \equiv y_{i_{1}} x_{i_{2}} \ldots x_{i_{l}}-y_{i_{1}} \ldots y_{i_{t}} x_{i_{t+1}} \ldots x_{i_{l}} \bmod \left(I_{S}\right)
$$

Como $y_{i_{1}} x_{i_{2}} x_{i_{3}}-y_{i_{1}} y_{i_{2}} x_{i_{3}} \in I_{S}$ tem-se que

$$
h_{i j t} \equiv y_{i_{1}}\left(y_{i_{2}} x_{i_{3}} \ldots x_{i_{l}}-y_{i_{2}} \ldots y_{i_{t}} x_{i_{t+1}} \ldots x_{i_{l}}\right) \bmod \left(I_{S}\right) .
$$

Observe que $y_{i_{2}} x_{i_{3}} \ldots x_{i_{l}}-y_{i_{2}} \ldots y_{i_{t}} x_{i_{t+1}} \ldots x_{i_{l}}$ se anula em $L \mathrm{e}$, por hipótese de indução, pertence a $I_{S}$, para todo $2 \leq t \leq l-1$. Segue que $h_{i j t}$ é gerado por polinômios em $I_{S}$, para todo $1 \leq t \leq l-1$. Portanto os polinômios homogêneos de grau $l$ em $I(L)$ pertencem a $I_{S}$. Segue pelo princípio da indução que $I_{S}=I(L)$. 
Devido à Proposição 3.2.1, $\Lambda_{\text {reg }}^{1}\left(\mathbb{R}^{2 n}\right)$ é gerado por 1-formas homogêneas. Primeiramente, encontraremos geradores de $\Lambda_{\text {reg }}^{1}\left(\mathbb{R}^{2 n}\right)$ homogêneos de grau até 4 . Posteriormente, no Lema 3.2.5, provaremos que elementos em $\Lambda_{\text {reg }}^{1}\left(\mathbb{R}^{2 n}\right)$ homogêneos de grau $\geq 5$ tem restrição algébrica a $L$ nula. Concluiremos que o espaço vetorial $\left[\Lambda_{\text {reg }}^{1}\left(\mathbb{R}^{2 n}\right)\right]_{L}$ tem dimensão finita e é gerado por restrições algébricas a $L$ de 1 -formas que se anulam em $T L_{\text {reg }}$ homogêneas de grau $\leq 4$.

Claramente não existe 1-formas homogêneas de grau 1 em $\Lambda_{\text {reg }}^{1}\left(\mathbb{R}^{2 n}\right)$.

\section{Geradores de grau 2:}

Seja $\gamma=\sum_{i, j=1}^{n}\left(a_{i j} x_{i} d x_{j}+b_{i j} x_{i} d y_{j}+c_{i j} y_{i} d x_{j}+e_{i j} y_{i} d y_{j}\right)$ uma 1-forma em $\Lambda_{\text {reg }}^{1}\left(\mathbb{R}^{2 n}\right)$. Como $\left.\gamma\right|_{L}=0$ segue que $\sum_{i, j=1}^{n} a_{i j} x_{i} d x_{j}=\sum_{i, j=1}^{n} e_{i j} y_{i} d y_{j}=0$ e $b_{i j}=-c_{j i}$, para todo $i, j \in\{1, \ldots, n\}$. Portanto as 1-formas homogêneas de grau 2 em $\Lambda_{\text {reg }}^{1}\left(\mathbb{R}^{2 n}\right)$ são combinações lineares das 1formas:

- $x_{i} d y_{j}-y_{i} d x_{j}, i, j \in\{1, \ldots, n\}$.

Analogamente encontramos os geradores de graus 3 e 4 .

\section{Geradores de grau 3:}

- $x_{i} x_{j} d y_{k}-x_{i} y_{j} d y_{k}$

- $x_{i} x_{j} d y_{k}-y_{i} x_{j} d x_{k}$

- $x_{i} x_{j} d y_{k}-y_{i} x_{j} d y_{k}$

- $x_{i} x_{j} d y_{k}-x_{i} y_{j} d x_{k}$

- $x_{i} x_{j} d y_{k}-y_{i} y_{j} d x_{k}$

onde $i, j, k \in\{1, \ldots, n\}$.

\section{Geradores de grau 4:}

- $x_{i} x_{j} x_{k} d y_{l}-x_{i} x_{j} y_{k} d y_{l}$

- $x_{i} x_{j} x_{k} d y_{l}-x_{i} y_{j} x_{k} d y_{l}$

- $x_{i} x_{j} x_{k} d y_{l}-y_{i} x_{j} x_{k} d y_{l}$

- $x_{i} x_{j} x_{k} d y_{l}-x_{i} y_{j} y_{k} d y_{l}$

- $x_{i} x_{j} x_{k} d y_{l}-y_{i} x_{j} y_{k} d y_{l}$

- $x_{i} x_{j} x_{k} d y_{l}-y_{i} y_{j} x_{k} d y_{l}$

- $x_{i} x_{j} x_{k} d y_{l}-x_{i} x_{j} y_{k} d x_{l}$
- $x_{i} x_{j} x_{k} d y_{l}-x_{i} y_{j} x_{k} d x_{l}$

- $x_{i} x_{j} x_{k} d y_{l}-y_{i} x_{j} x_{k} d x_{l}$

- $x_{i} x_{j} x_{k} d y_{l}-x_{i} y_{j} y_{k} d x_{l}$

- $x_{i} x_{j} x_{k} d y_{l}-y_{i} x_{j} y_{k} d x_{l}$

- $x_{i} x_{j} x_{k} d y_{l}-y_{i} y_{j} x_{k} d x_{l}$

- $x_{i} x_{j} x_{k} d y_{l}-y_{i} y_{j} y_{k} d x_{l}$

onde $i, j, k, l \in\{1, \ldots, n\}$. 
Lema 3.2.5. As 1-formas homogêneas de grau maior ou igual a $5 \mathrm{em} \Lambda_{\mathrm{reg}}^{1}\left(\mathbb{R}^{2 n}\right)$ têm restrição algébrica a L nula.

Demonstração. Seja $\tilde{\gamma}=\sum_{j=1}^{n}\left(f_{j}(x, y) d x_{i}+g_{j}(x, y) d y_{i}\right)$ em $\Lambda_{\text {reg }}^{1}\left(\mathbb{R}^{2 n}\right)$, onde $f_{j}, g_{j}$ são germes de funções em $\left(\mathbb{R}^{2 n}, 0\right), i=1, \ldots, n$. Como $\tilde{\gamma}$ se anula em $T L_{1}$ e em $T L_{2}$ segue que $\tilde{\gamma}=$ $\sum_{i, j=1}^{n}\left(y_{i} f_{i j}(x, y) d x_{i}+x_{i} g_{i j}(x, y) d y_{j}\right)$, onde $f_{i j}, g_{i j}$ são germes de funções em $\left(\mathbb{R}^{2 n}, 0\right), i, j=$ $1, \ldots, n$.

Seja $\gamma$ uma 1-forma homogênea de grau $l+1 \geq 5$ em $\Lambda_{\text {reg }}^{1}\left(\mathbb{R}^{2 n}\right)$, então $\gamma$ é da forma:

$$
\begin{aligned}
\gamma= & \sum a_{i_{1} \cdots i_{l} k}^{(1)} y_{i_{1}} x_{i_{2}} \cdots x_{i_{l}} d x_{k}+\cdots+\sum a_{i_{1} \cdots i_{l} k}^{(l)} y_{i_{1}} \cdots y_{i_{l}} d x_{k} \\
& \sum b_{i_{1} \cdots i_{l} k}^{(1)} x_{i_{1}} y_{i_{2}} \cdots y_{i_{l}} d y_{k}+\cdots+\sum b_{i_{1} \cdots i_{l} k}^{(l)} x_{i_{1}} \cdots x_{i_{l}} d y_{k},
\end{aligned}
$$

onde $i_{1}, \ldots, i_{l}, k \in\{1, \ldots, n\}$. Como $\left.\gamma\right|_{T L_{3}}=0$, para $i_{1}, \ldots, i_{l}, k \in\{1, \ldots, n\}$ tem-se

$$
\sum_{\sigma \in S_{l}} a_{\sigma\left(i_{1}\right) \cdots \sigma\left(i_{l}\right) k}^{(1)}+\cdots+\sum_{\sigma \in S_{l}} a_{\sigma\left(i_{1}\right) \cdots \sigma\left(i_{l}\right) k}^{(l)}+\sum_{\sigma \in S_{l}} b_{\sigma\left(i_{1}\right) \cdots \sigma\left(i_{l}\right) k}^{(1)}+\cdots+\sum_{\sigma \in S_{l}} b_{\sigma\left(i_{1}\right) \cdots \sigma\left(i_{l}\right) k}^{(l)}=0
$$

onde $S_{l}$ é o grupo das permutações de $\left\{i_{1}, \ldots, i_{l}\right\}$. Portanto as 1-formas homogêneas de grau $l+1$ em $\Lambda_{\text {reg }}^{1}\left(\mathbb{R}^{2 n}\right)$ são geradas por 1-formas do tipo

$$
\begin{aligned}
& \rho_{t \sigma}=y_{i_{1}} y_{i_{2}} x_{i_{3}} \cdots x_{i_{l}} d x_{k}-x_{\sigma\left(i_{1}\right)} \cdots x_{\sigma\left(i_{t}\right)} y_{\sigma\left(i_{t+1}\right)} \cdots y_{\sigma\left(i_{l}\right)} d x_{k}, \\
& \xi_{t \sigma}=y_{i_{1}} y_{i_{2}} x_{i_{3}} \cdots x_{i_{l}} d x_{k}-y_{\sigma\left(i_{1}\right)} \cdots y_{\sigma\left(i_{t}\right)} x_{\sigma\left(i_{t+1}\right)} \cdots x_{\sigma\left(i_{l}\right)} d y_{k},
\end{aligned}
$$

onde $\sigma \in S_{l}$ e $0 \leq t \leq l-1$.

Seja $t \in\{1, \ldots, l-1\}$. Observe que o polinômio $y_{i_{1}} y_{i_{2}} x_{i_{3}} \cdots x_{i_{l}} x_{k}-$ $x_{\sigma\left(i_{1}\right)} \cdots x_{\sigma\left(i_{t}\right)} y_{\sigma\left(i_{t+1}\right)} \cdots y_{\sigma\left(i_{l}\right)} x_{k}$ se anula em $L$. Portanto as 1-formas do tipo $\rho_{t \sigma}$ e $\xi_{t \sigma}$ são gerados por

$$
\begin{aligned}
& \rho=y_{i_{1}} y_{i_{2}} x_{i_{3}} \cdots x_{i_{l}} d x_{k}-y_{i_{1}} \cdots y_{i_{l}} d x_{k} \\
& \xi_{1}=y_{i_{1}} y_{i_{2}} x_{i_{3}} \cdots x_{i_{l}} d x_{k}-x_{i_{1}} \cdots x_{i_{l}} d y_{k} \\
& \xi_{2}=y_{i_{1}} y_{i_{2}} x_{i_{3}} \cdots x_{i_{l}}\left(d x_{k}-d y_{k}\right) .
\end{aligned}
$$

Observe que o polinômio $h(x, y)=y_{i_{1}} y_{i_{2}} x_{i_{3}} \cdots x_{i_{l}}\left(x_{k}-y_{k}\right)$ se anula em $L$, logo a 1-forma $d h$ tem restrição algébrica a $L$ nula. Temos que $d h=\xi_{2}+\hat{\gamma}$, onde 


$$
\begin{aligned}
\hat{\gamma} & =y_{i_{2}} x_{i_{3}} \cdots x_{i_{l}}\left(x_{k}-y_{k}\right) d y_{i_{1}}+y_{i_{1}} x_{i_{3}} \cdots x_{i_{l}}\left(x_{k}-y_{k}\right) d y_{i_{2}} \\
& +\sum_{u=3}^{l} y_{i_{1}} y_{i_{2}} x_{i_{3}} \cdots x_{i_{u-1}} x_{i_{u+1}} \cdots x_{i_{l}}\left(x_{k}-y_{k}\right) d x_{u}
\end{aligned}
$$

Claramente $\hat{\gamma}$ tem restrição algébrica a $L$ nula. Portanto $\xi_{2}$ tem restrição algébrica a $L$ nula. A prova que 1-formas do tipo $\rho$ e $\xi_{1}$ tem restrição algébrica a $L$ nula segue diretamente do fato de que $\xi_{2}$ tem restrição algébrica a $L$ nula.

Encontrar uma base do espaço vetorial $\left[\Lambda_{\text {reg }}^{2, c l}\left(\mathbb{R}^{2 n}\right)\right]_{L}$ é um problema difícil. O seguinte resultado fornece um conjunto finito de geradores de $\left[\Lambda_{\mathrm{reg}}^{2, \mathrm{cl}}\left(\mathbb{R}^{2 n}\right)\right]_{L}$.

Proposição 3.2.6. Um conjunto finito de geradores de $\left[\Lambda_{\mathrm{reg}}^{2, c l}\left(\mathbb{R}^{2 n}\right)\right]_{L}$ é dado por:

- Grau 2: $\left[d x_{i} \wedge d y_{j}-d y_{i} \wedge d x_{j}\right]_{L}, \quad 1 \leq i \leq j \leq n$;

- Grau 3: $\left[d\left(x_{i} y_{j}\right) \wedge d x_{k}-d\left(y_{i} y_{j}\right) \wedge d x_{k}\right]_{L}, \quad\left[d\left(x_{i} y_{j}\right) \wedge d x_{k}-d\left(x_{i} y_{j}\right) \wedge d y_{k}\right]_{L}, \quad 1 \leq i \leq j \leq$ $n, 1 \leq k \leq n$;

- Grau 4: $\left[d\left(x_{i} x_{j} y_{k}\right) \wedge d x_{l}-d\left(y_{i} y_{j} x_{k}\right) \wedge d y_{l}\right]_{L}, 1 \leq i \leq j \leq k \leq n, 1 \leq l \leq n$.

Demonstração. De acordo com as Proposições 3.2.2 e 3.2.3, as derivadas dos geradores de $\left[\Lambda_{\text {reg }}^{1}\left(\mathbb{R}^{2 n}\right)\right]_{L}$ geram $\left[\Lambda_{\text {reg }}^{2, \mathrm{cl}}\left(\mathbb{R}^{2 n}\right)\right]_{L}$. Sendo assim, é suficiente verificar que

- $x_{i} d y_{j}-y_{i} d x_{j}, 1 \leq i \leq j \leq n$

- $x_{i} y_{j} d x_{k}-y_{i} y_{j} d x_{k}, x_{i} y_{j} d x_{k}-x_{i} y_{j} d y_{k} 1 \leq i \leq j \leq n, 1 \leq k \leq n$

- $x_{i} x_{j} y_{k} d x_{l}-y_{i} y_{j} x_{k} d y_{l}, 1 \leq i \leq j \leq k \leq n, 1 \leq l \leq n$.

geram $\left[\Lambda_{\text {reg }}^{1}\left(\mathbb{R}^{2 n}\right)\right]_{L}$. De acordo com a Proposição 3.2.1, fixamos um grau e encontramos geradores para este grau. Como a prova é bem similar para cada grau fixado, encontraremos um conjunto de geradores homogêneos de grau 4. Sabemos que as 1-formas homogêneas de grau 4 são geradas por:

- $x_{i} x_{j} x_{k} d y_{l}-x_{i} x_{j} y_{k} d y_{l}$

- $x_{i} x_{j} x_{k} d y_{l}-x_{i} y_{j} x_{k} d y_{l}$

- $x_{i} x_{j} x_{k} d y_{l}-y_{i} x_{j} x_{k} d y_{l}$

- $x_{i} x_{j} x_{k} d y_{l}-x_{i} y_{j} y_{k} d y_{l}$

- $x_{i} x_{j} x_{k} d y_{l}-y_{i} x_{j} y_{k} d y_{l}$
- $x_{i} x_{j} x_{k} d y_{l}-y_{i} y_{j} x_{k} d y_{l}$

- $x_{i} x_{j} x_{k} d y_{l}-x_{i} x_{j} y_{k} d x_{l}$

- $x_{i} x_{j} x_{k} d y_{l}-x_{i} y_{j} x_{k} d x_{l}$

- $x_{i} x_{j} x_{k} d y_{l}-y_{i} x_{j} x_{k} d x_{l}$

- $x_{i} x_{j} x_{k} d y_{l}-x_{i} y_{j} y_{k} d x_{l}$ 
- $x_{i} x_{j} x_{k} d y_{l}-y_{i} x_{j} y_{k} d x_{l}$

- $x_{i} x_{j} x_{k} d y_{l}-y_{i} y_{j} y_{k} d x_{l}$

- $x_{i} x_{j} x_{k} d y_{l}-y_{i} y_{j} x_{k} d x_{l}$

onde $i, j, k, l \in\{1, \ldots, n\}$. A menos de soma de restrições algébricas a $L$ nulas da forma $\left[h(x, y) d x_{l}\right]_{L}$ e $\left[h(x, y) d y_{l}\right]_{L}$, onde $h \in I(L)$, reduzimos os geradores de grau 4 aos seguintes:

- $\left[x_{i} x_{j} x_{k} d y_{l}-x_{i} x_{j} y_{k} d y_{l}\right]_{L}$

- $\left[x_{i} x_{j} x_{k} d y_{l}-x_{i} y_{j} y_{k} d x_{l}\right]_{L}$

- $\left[x_{i} x_{j} x_{k} d y_{l}-y_{i} y_{j} y_{k} d x_{l}\right]_{L}$

onde $i, j, k, l \in\{1, \ldots, n\}$. Observe que $\gamma_{1}=d\left(x_{i} x_{j} x_{k} y_{l}-x_{i} x_{j} y_{k} y_{l}\right) \in \mathscr{A}_{0}^{1}\left(L,\left(\mathbb{R}^{2 n}, 0\right)\right)$. Além disso,

$$
\begin{aligned}
d\left(x_{i} x_{j} x_{k} y_{l}-x_{i} x_{j} y_{k} y_{l}\right)= & \left(x_{j} x_{k} y_{l}-x_{j} y_{k} y_{l}\right) d x_{i}+\left(x_{i} x_{k} y_{l}-x_{i} y_{k} y_{l}\right) d x_{j}+ \\
& x_{i} x_{j} y_{l} d x_{k}-x_{i} x_{j} y_{l} d y_{k}+\left(x_{i} x_{j} x_{k}-x_{i} x_{j} y_{k}\right) d y_{l} .
\end{aligned}
$$

Logo as restrições algébricas a $L$ da forma $\left[x_{i} x_{j} x_{k} d y_{l}-x_{i} x_{j} y_{k} d y_{l}\right]_{L}$ são geradas por restrições algébricas a $L$ da forma $\left[x_{i} x_{j} y_{k} d y_{l}-y_{i} y_{j} x_{k} d x_{l}\right]_{L}, i, j, k, l \in\{1, \ldots, n\}$. Analogamente, $\left[x_{i} x_{j} x_{k} d y_{l}-x_{i} y_{j} y_{k} d x_{l}\right]_{L}$ e $\left[x_{i} x_{j} x_{k} d y_{l}-y_{i} y_{j} y_{k} d x_{l}\right]_{L}$ são gerados por $\left[x_{i} x_{j} y_{k} d y_{l}-y_{i} y_{j} x_{k} d x_{l}\right]_{L}$, $i, j, k, l \in\{1, \ldots, n\}$.

As restrições algébricas a $L$

$$
\left[x_{i} x_{j} y_{k} d x_{l}-y_{i} y_{j} x_{k} d y_{l}\right]_{L}, 1 \leq i \leq j \leq k \leq n, 1 \leq l \leq n,
$$

geram as restrições algébricas a $L$ das 1 -formas homogêneas de grau 4 em $\left[\Lambda_{\text {reg }}^{1}\left(\mathbb{R}^{2 n}\right)\right]_{L}$ pois para qualquer permutação dos índices $i, j, k$ as restrições algébricas a $L$ da forma $\left[x_{i} x_{j} y_{k} d x_{l}-\right.$ $\left.y_{i} y_{j} x_{k} d y_{l}\right]_{L}$ são iguais.

\subsection{Redução ao caso linear}

Nesta seção reduzimos a classificação das restrições algébricas a $L$ de formas simpléticas que se anulam em $T L_{\text {reg }}$ sob a ação das simetrias locais de $L$ à classificação de restrições algébricas a $L$ de formas simpléticas homogêneas de grau 2 sob a ação das simetrias locais de $L$ lineares.

Definição 3.3.1. Um germe de campo de vetores $\eta \mathrm{em}\left(\mathbb{R}^{m}, 0\right)$ é levantável sobre um multigerme $F=$ $\left\{F_{1}, \ldots, F_{s}\right\}:\left(\mathbb{R}^{k}, 0\right) \rightarrow\left(\mathbb{R}^{m}, 0\right)$ se existem germes de campos de vetores $\xi_{1}, \ldots, \xi_{s}$ em $\left(\mathbb{R}^{k}, 0\right)$ tais que 


$$
d F_{i} \circ \xi_{i}=\eta \circ F_{i}, i=1, \ldots, s .
$$

Denotamos o conjunto dos germes dos campos levantáveis de $F$ por $\operatorname{Lift}(F)$.

Considere o multigerme $F:\left\{F_{1}, F_{2}, F_{3}\right\}:\left(\mathbb{R}^{n}, 0\right) \rightarrow\left(\mathbb{R}^{2 n}, 0\right)$ definido por $F_{1}\left(x_{1}, \ldots, x_{n}\right)=$ $\left(x_{1}, \ldots, x_{n}, 0, \ldots, 0\right), \quad F_{2}\left(y_{1}, \ldots, y_{n}\right)=\left(0, \ldots, 0, y_{1}, \ldots, y_{n}\right)$ e $F_{3}\left(z_{1}, \ldots, z_{n}\right)=$ $\left(z_{1}, \ldots, z_{n}, z_{1}, \ldots, z_{n}\right)$. Observe que a imagem do multigerme $F$ é $L$.

Proposição 3.3.2. Os germes de campos de vetores levantáveis sobre $F$ são da forma $\sum_{i=1}^{n}\left(X_{i} \frac{\partial}{\partial x_{i}}+Y_{i} \frac{\partial}{\partial y_{i}}\right)$, onde

$$
\begin{aligned}
& X_{i} \in\left\langle x_{1}, \ldots, x_{n}\right\rangle \\
& Y_{i} \in\left\langle y_{1}, \ldots, y_{n}\right\rangle \\
& X_{i}-Y_{i} \in\left\langle x_{1}-y_{1}, \ldots, x_{n}-y_{n}\right\rangle,
\end{aligned}
$$

$i=1, \ldots, n$.

Demonstração. Seja $\eta=\sum_{i=1}^{n}\left(X_{i} \frac{\partial}{\partial x_{i}}+Y_{i} \frac{\partial}{\partial y_{i}}\right)$ um germe de campo de vetores em $\left(\mathbb{R}^{2 n}, 0\right)$ como no enunciado. Então

$$
\begin{aligned}
\eta \circ F_{1}(x) & =\left(X_{1}(x, 0), \ldots, X_{n}(x, 0), 0, \ldots, 0\right) \\
\eta \circ F_{2}(y) & =\left(0, \ldots, 0, Y_{1}(0, y), \ldots, Y_{n}(0, y)\right) \\
\eta \circ F_{3}(z) & =\left(X_{1}(z, z), \ldots, X_{n}(z, z), Y_{1}(z, z), \ldots, Y_{n}(z, z)\right) \\
& =\left(X_{1}(z, z), \ldots, X_{n}(z, z), X_{1}(z, z), \ldots, X_{n}(z, z)\right) .
\end{aligned}
$$

Considere os germes de campo de vetores

$$
\begin{aligned}
& \xi_{1}(x)=\sum_{i=1}^{n} X_{i}(x, 0) \frac{\partial}{\partial x_{i}} \\
& \xi_{2}(y)=\sum_{i=1}^{n} Y_{i}(0, y) \frac{\partial}{\partial y_{i}} \\
& \xi_{3}(z)=\sum_{i=1}^{n} X_{i}(z, z) \frac{\partial}{\partial z_{i}} .
\end{aligned}
$$

Observe que 


$$
d F_{i} \circ \xi_{i}=\eta \circ F_{i}, \quad i=1,2,3 .
$$

Segue $\eta$ é levantável sobre $F$.

Seja $W=\sum_{i=1}^{n}\left(U_{i} \frac{\partial}{\partial x_{i}}+V_{i} \frac{\partial}{\partial y_{i}}\right) \in \operatorname{Lift}(F)$. Sendo assim, existem $\rho_{1}, \rho_{2}, \rho_{3}$ germes de campo de vetores em $\mathbb{R}^{n}$ tais que

$$
W \circ F_{i}=d F_{i} \circ \rho_{i} .
$$

Escrevendo $\rho_{1}$ como $\rho_{1}(x)=\sum_{j=1}^{n} \rho_{1}^{j}(x) \frac{\partial}{\partial x_{j}}$ tem-se

$$
d F_{1}(x) \cdot \rho_{1}(x)=\left(\rho_{1}^{1}(x), \ldots, \rho_{1}^{n}(x), 0, \ldots, 0\right) .
$$

Logo $V_{i} \in\left\langle y_{1}, \ldots, y_{n}\right\rangle$, para todo $i \in\{1, \ldots, n\}$. Segue analogamente que $U_{i} \in\left\langle x_{1}, \ldots, x_{n}\right\rangle$ e $U_{i}-V_{i} \in\left\langle x_{1}-y_{1}, \ldots, x_{n}-y_{n}\right\rangle$, para todo $i=1, \ldots, n$.

O próximo resultado estabelece uma relação entre germes de campos levantáveis e tangentes.

Proposição 3.3.3. Se $\eta \in \operatorname{Lift}(F)$ então $\eta$ é tangente a $L$.

Demonstração. Seja $h$ um germe de função que se anula em $L$. Por hipótese, existem germes de campo de vetores $\xi_{i}$ tais que $\eta \circ F_{i}=d F_{i} \circ \xi_{i}, i=1,2,3$. Se $p \in\left(\mathbb{R}^{n}, 0\right)$ então

$$
\begin{aligned}
(d h \circ \eta)\left(F_{i}(p)\right) & =d h\left(F_{i}(p)\right) \cdot \eta\left(F_{i}(p)\right)=d h\left(F_{i}(p)\right) \cdot d F_{i}(p) \cdot \xi_{i}(p) \\
& =d\left(h \circ F_{i}\right)(p) \cdot \xi_{i}(p)=0,
\end{aligned}
$$

pois $h \circ F_{i} \equiv 0 \mathrm{em}\left(\mathbb{R}^{n}, 0\right)$.

Proposição 3.3.4. Sejam $\eta \in \operatorname{Lift}(F) e \theta \in \mathscr{A}_{0}^{k}\left(L,\left(\mathbb{R}^{2 n}, 0\right)\right)$. Então $\mathcal{L}_{\eta} \theta \in \mathscr{A}_{0}^{k}\left(L,\left(\mathbb{R}^{2 n}, 0\right)\right)$.

Demonstração. Segue diretamente do fato de que $\eta$ é tangente a $L$ e $\mathcal{L}_{\eta}(d \beta)=d\left(\mathcal{L}_{\eta} \beta\right)$, para toda $(k-1)$-forma $\beta$.

Proposição 3.3.5. Sejam $\sigma \in \Lambda_{\text {reg }}^{2, c l}\left(\mathbb{R}^{2 n}\right)$ e $\eta \in \operatorname{Lift}(F)$ então $\mathcal{L}_{\eta} \sigma \in \Lambda_{\text {reg }}^{2, c l}\left(\mathbb{R}^{2 n}\right)$.

Demonstração. Pela Proposição 3.2.2 existe uma 1-forma $\gamma \in \Lambda_{\text {reg }}^{1}\left(\mathbb{R}^{2 n}\right)$ tal que $\sigma=d \gamma$. Portanto, $\mathcal{L}_{\eta} \sigma=\mathcal{L}_{\eta} d \gamma=d \mathcal{L}_{\eta} \gamma$. Pela Proposição 3.2.3, é suficiente provar que $\mathcal{L}_{\eta} \gamma$ se anula em $T L_{\text {reg. }}$.

Sejam $\gamma=\sum_{j=1}^{n}\left(f_{j}(x, y) d x_{j}+g_{j}(x, y) d y_{j}\right) \in \Lambda_{\text {reg }}^{1}\left(\mathbb{R}^{2 n}\right)$ e $X_{i}, Y_{i}$ germes de funções reais, $i=1, \ldots, n$, tais que $\eta=\sum_{j=1}^{n}\left(X_{j} \frac{\partial}{\partial x_{i}}+Y_{j} \frac{\partial}{\partial y_{i}}\right)$. Como $\gamma$ se anula em $T L_{\text {reg }}$ temos que $f_{j}(x, 0)=0, g_{j}(0, y)=0$ e $\left(f_{j}+g_{j}\right)(z, z)=0$. Então 


$$
\begin{aligned}
& \mathcal{L}_{\eta} \gamma=d\left(i_{\eta} \gamma\right)+i_{\eta}(d \gamma) \\
& =d\left(\sum_{j=1}^{n}\left(f_{j} X_{j}+g_{j} Y_{j}\right)\right)+i_{\eta}\left(d\left(\sum_{j=1}^{n}\left(f_{j} d x_{j}+g_{j} d y_{j}\right)\right)\right) \\
& =\sum_{i, j=1}^{n}\left(\frac{\partial\left(f_{j} X_{j}\right)}{\partial x_{i}} d x_{i}+\frac{\partial\left(f_{j} X_{j}\right)}{\partial y_{i}} d y_{i}+\frac{\partial\left(g_{j} Y_{j}\right)}{\partial x_{i}} d x_{i}+\frac{\partial\left(g_{j} Y_{j}\right)}{\partial y_{i}} d y_{i}\right) \\
& +\sum_{i, j=1}^{n}\left(\frac{\partial f_{j}}{\partial x_{i}}\left(X_{i} d x_{j}-X_{j} d x_{i}\right)+\frac{\partial f_{j}}{\partial y_{i}}\left(Y_{i} d x_{j}-X_{j} d y_{i}\right)\right) \\
& +\sum_{i, j=1}^{n}\left(\frac{\partial g_{j}}{\partial x_{i}}\left(X_{i} d y_{j}-Y_{j} d x_{i}\right)+\frac{\partial g_{j}}{\partial y_{i}}\left(Y_{i} d y_{j}-Y_{j} d y_{i}\right)\right)
\end{aligned}
$$

Segue da Proposição 3.3.2 que $\mathcal{L}_{\eta} \gamma$ se anula em $T L_{1}$ e em $T L_{2}$. A restrição de $\mathcal{L}_{\eta} \gamma$ a $T L_{3}$ é zero pois

$$
\begin{aligned}
& \left.\mathcal{L}_{\eta} \gamma\right|_{L_{3}}= \\
& =\sum_{i, j=1}^{n}\left(\frac{\partial\left(f_{j} Z_{j}\right)}{\partial z_{i}}(z, z) d z_{i}+\frac{\partial\left(g_{j} Z_{j}\right)}{\partial z_{i}}(z, z) d z_{i}\right) \\
& +\sum_{i, j=1}^{n}\left(\frac{\partial f_{j}}{\partial z_{i}}(z, z)\left(Z_{i} d z_{j}-Z_{j} d z_{i}\right)+\frac{\partial g_{j}}{\partial z_{i}}(z, z)\left(Z_{i} d z_{j}-Z_{j} d z_{i}\right)\right) \\
& =\sum_{i, j=1}^{n}\left(\frac{\partial\left(\left(f_{j}+g_{j}\right) Z_{j}\right)}{\partial z_{i}}(z, z) d z_{i}+\frac{\partial\left(f_{j}+g_{j}\right)}{\partial z_{i}}(z, z)\left(Z_{i} d z_{j}-Z_{j} d z_{i}\right)\right)
\end{aligned}
$$

onde $Z_{j}(z)=X_{j}(z, z)=Y_{j}(z, z), j=1, \ldots, n$.

Seja $a \in\left[\operatorname{Symp}_{\text {reg }}\left(\mathbb{R}^{2 n}\right)\right]_{L}$. Devido à Proposição 3.2.6, a restrição algébrica a $L$ a tem um representante simplético $\sigma=\sigma^{(2)}+\sigma^{(3)}+\sigma^{(4)}$ onde 


$$
\begin{aligned}
\sigma^{(2)}= & \sum_{\substack{1 \leq i \leq j \leq n \\
\sigma^{(3)}=}} a_{i j}\left(d x_{i} \wedge d y_{j}-d y_{i} \wedge d x_{j}\right) \\
& \sum_{\substack{1 \leq i \leq j \leq n, 1 \leq k \leq n}} b_{i j k}^{(1)}\left(d\left(x_{i} y_{j}\right) \wedge d x_{k}-d\left(y_{i} y_{j}\right) \wedge d x_{k}\right)+ \\
& \sum_{\substack{1 \leq i \leq j \leq n, 1 \leq k \leq n}} b_{i j k}^{(2)}\left(d\left(x_{i} y_{j}\right) \wedge d x_{k}-d\left(x_{i} y_{j}\right) \wedge d y_{k}\right) \\
\sigma^{(4)}= & \sum_{\substack{1 \leq i \leq j \leq k \leq n \\
1 \leq l \leq n}} c_{i j k l}\left(d\left(x_{i} x_{j} y_{k}\right) \wedge d x_{l}-d\left(y_{i} y_{j} x_{k}\right) \wedge d y_{l}\right) .
\end{aligned}
$$

onde $a_{i j}, b_{i j k}^{(1)}, b_{i j k}^{(2)}, c_{i j k l} \in \mathbb{R}$. A 2-forma fechada $\sigma$ é simplética se, e somente se, $\sigma(0)$ é uma forma bilinear simplética. Observe que $\sigma(0)$ tem matriz associada

$$
M=\left[\begin{array}{cc}
0 & C \\
-C & 0
\end{array}\right]
$$

onde $C=\left(c_{i j}\right) \in M(n, \mathbb{R})$ definida por $C=\left\{\begin{array}{l}c_{i j}=a_{i j}, i<j \\ c_{i j}=a_{j i}, i>j \\ c_{i i}=2 a_{i i}, i=1, \ldots, n .\end{array}\right.$.

Portanto $\sigma(0)$ é simplética se, e somente se, $C \in \mathrm{GL}(n, \mathbb{R})$.

Proposição 3.3.6. A restrição algébrica $[\sigma]_{L}$ é difeomorfa a $\left[\sigma^{(2)}\right]_{L}$.

A demonstração da Proposição 3.3.6 será feita nos próximos 2 Lemas.

Lema 3.3.7. A restrição algébrica $[\sigma]_{L}$ é difeomorfa $a\left[\sigma^{(2)}+\theta\right]_{L}$, onde $\theta$ é uma 2-forma homogênea de grau 4 que se anula em $T L_{\mathrm{reg}}$.

Demonstração. Para demonstrar usamos o método de homotopia de Moser como em [D1].

Seja

$$
\sigma_{t}^{(4)}=\sum_{\substack{1 \leq i \leq j \leq k \leq n \\ 1 \leq l \leq n}} f_{i j k l}(t)\left(d\left(x_{i} x_{j} y_{k}\right) \wedge d x_{l}-d\left(y_{i} y_{j} x_{k}\right) \wedge d y_{l}\right),
$$

onde $f_{i j k l}:[0,1] \rightarrow \mathbb{R}$ são germes de funções com $f_{i j k l}(0)=c_{i j k l}, 1 \leq i \leq j \leq k \leq n$ e $1 \leq l \leq n$. Seja $\sigma_{t}=\sigma^{(2)}+(1-t) \sigma^{(3)}+\sigma_{t}^{(4)}$. Suponhamos que existe $\Phi_{t}:\left(\mathbb{R}^{2 n}, 0\right) \rightarrow\left(\mathbb{R}^{2 n}, 0\right), t \in[0,1]$, uma família de simetrias locais de $L$ tal que

$$
\Phi_{t}^{*}\left[\sigma_{t}\right]_{L}=[\sigma]_{L} \text { e } \Phi_{0}=I d .
$$


Segue da Proposição 1.1.21 que derivando (3.1) em relação a $t$ obtemos

$$
\Phi_{t}^{*}\left[\mathcal{L}_{\eta_{t}} \sigma_{t}-\sigma^{(3)}+\frac{d \sigma_{t}^{(4)}}{d t}\right]_{L}=0
$$

onde $\eta_{t}$ satisfaz a equação $d \Phi_{t} / d t=\eta_{t} \circ \Phi_{t}$. Logo

$$
\left[\mathcal{L}_{\eta_{t}} \sigma_{t}\right]_{L}=\left[\sigma^{(3)}-\frac{d \sigma_{t}^{(4)}}{d t}\right]_{L}
$$

Se encontrarmos um germe de campo de vetores $\eta_{t}$ levantável sobre $F$ satisfazendo (3.2) então seu fluxo satisfaz (3.1).

De acordo com a Proposição 1.2.18, queremos encontrar um germe de campo de vetores $\eta_{t} \in \operatorname{Lift}(F)$ homogêneo de grau 1 que satisfaz

$$
\mathcal{L}_{\eta_{t}} \sigma^{(2)}=\sigma^{(3)}
$$

Segue da fórmula de Cartan que

$$
\begin{aligned}
d\left(i_{\eta_{t}} \sigma^{(2)}\right)= & \sum_{\substack{1 \leq i \leq j \leq n, 1 \leq k \leq n}}\left(b_{i j k}^{(1)}\left(d\left(x_{i} y_{j}\right) \wedge d x_{k}-d\left(y_{i} y_{j}\right) \wedge d x_{k}\right)+\right. \\
& b_{i j k}^{(2)}\left(d\left(x_{i} y_{j}\right) \wedge d x_{k}-d\left(x_{i} y_{j}\right) \wedge d y_{k}\right) .
\end{aligned}
$$

Portanto se encontrarmos um germe de campo de vetores $\eta_{t}$ que satisfaz

$$
\begin{aligned}
i_{\eta_{t}} \sigma^{(2)}= & \sum_{\substack{1 \leq i \leq j \leq n, 1 \leq k \leq n}}\left(b_{i j k}^{(1)}\left(x_{i} y_{j} d x_{k}-y_{i} y_{j} d x_{k}\right)+\right. \\
& \left.b_{i j k}^{(2)}\left(x_{i} y_{j} d x_{k}-x_{i} y_{j} d y_{k}\right)\right)
\end{aligned}
$$

consequentemente tal germe de campo de vetores irá satisfazer (3.3).

$$
\text { Se } \eta_{t}=\sum_{i=1}^{n}\left(X_{i}(t, x, y) \frac{\partial}{\partial x_{i}}+Y_{i}(t, x, y) \frac{\partial}{\partial y_{i}}\right) \text { então }
$$




$$
\begin{aligned}
i_{\eta_{t}} \sigma^{(2)} & =\sum_{1 \leq i \leq j \leq n} a_{i j}\left(X_{i} d y_{j}-Y_{j} d x_{i}+X_{j} d y_{i}-Y_{i} d x_{j}\right) \\
& =\sum_{i, j=1}^{n} e_{i j}\left(X_{i} d y_{j}-Y_{i} d x_{j}\right),
\end{aligned}
$$

onde $\left\{\begin{array}{l}e_{i j}=a_{i j}, i<j \\ e_{i j}=a_{j i}, i>j \\ e_{i i}=2 a_{i i}, i=1, \ldots, n\end{array}\right.$. Observe que $E=\left(e_{i j}\right)=C \in \operatorname{GL}(n, \mathbb{R})$.

Portanto $\eta_{t}$ deve satisfazer

$$
\begin{aligned}
\sum_{i, j=1}^{n} e_{i j}\left(X_{i} d y_{j}-Y_{i} d x_{j}\right)= & \sum_{\substack{1 \leq i \leq j \leq n, 1 \leq k \leq n}}\left(b_{i j k}^{(1)}\left(x_{i} y_{j} d x_{k}-y_{i} y_{j} d x_{k}\right)+\right. \\
& \left.b_{i j k}^{(2)}\left(x_{i} y_{j} d x_{k}-x_{i} y_{j} d y_{k}\right)\right) .
\end{aligned}
$$

Temos o seguinte sistema

$$
\left\{\begin{array}{l}
\sum_{s=1}^{n} e_{s k} Y_{s}=-\sum_{1 \leq i \leq j \leq n}\left(b_{i j k}^{(1)}\left(x_{i} y_{j}-y_{i} y_{j}\right)+b_{i j k}^{(2)} x_{i} y_{j}\right) \\
\sum_{s=1}^{n} e_{s k} X_{s}=-\sum_{1 \leq i \leq j \leq n} b_{i j k}^{(2)} x_{i} y_{j},
\end{array}\right.
$$

onde $k=1, \ldots, n$.

Temos que o sistema se escreve como

$$
\left(\begin{array}{cc}
E & 0 \\
0 & E
\end{array}\right)\left(\begin{array}{c}
X_{1} \\
\vdots \\
X_{n} \\
Y_{1} \\
\vdots \\
Y_{n}
\end{array}\right)=\sum_{1 \leq i \leq j \leq n}\left(\begin{array}{c}
-b_{i j 1}^{(2)} x_{i} y_{j} \\
\vdots \\
-b_{i j n}^{(2)} x_{i} y_{j} \\
-b_{i j 1}^{(1)}\left(x_{i} y_{j}-y_{i} y_{j}\right)-b_{i j 1}^{(2)} x_{i} y_{j} \\
\vdots \\
-b_{i j n}^{(1)}\left(x_{i} y_{j}-y_{i} y_{j}\right)-b_{i j n}^{(2)} x_{i} y_{j}
\end{array}\right)
$$

Seja $W=\left(w_{i j}\right) \in \operatorname{GL}(n, \mathbb{R})$ a matriz inversa de $E$. A solução do sistema é dada por 


$$
\begin{aligned}
& X_{1}=-\sum_{1 \leq i \leq j \leq n}\left(w_{11}\left(b_{i j 1}^{(2)} x_{i} x_{j}\right)+\cdots+w_{1 n}\left(b_{i j n}^{(2)} x_{i} x_{j}\right)\right) \\
& X_{n}=-\sum_{1 \leq i \leq j \leq n}\left(w_{n 1}\left(b_{i j 1}^{(2)} x_{i} x_{j}\right)+\cdots+w_{n n}\left(b_{i j n}^{(2)} x_{i} x_{j}\right)\right) \\
& Y_{1}=-\sum_{1 \leq i \leq j \leq n}\left(w_{11}\left(b_{i j 1}^{(1)}\left(x_{i} y_{j}-y_{i} y_{j}\right)+b_{i j 1}^{(2)} x_{i} y_{j}\right)+\cdots+\right. \\
& \left.w_{1 n}\left(b_{i j n}^{(1)}\left(x_{i} y_{j}-y_{i} y_{j}\right)+b_{i j n}^{(2)} x_{i} y_{j}\right)\right) \\
& Y_{n}=-\sum_{1 \leq i \leq j \leq n}\left(w_{n 1}\left(b_{i j 1}^{(1)}\left(x_{i} y_{j}-y_{i} y_{j}\right)+b_{i j 1}^{(2)} x_{i} y_{j}\right)+\cdots+\right. \\
& \left.w_{n n}\left(b_{i j n}^{(1)}\left(x_{i} y_{j}-y_{i} y_{j}\right)+b_{i j n}^{(2)} x_{i} y_{j}\right)\right) .
\end{aligned}
$$

Pela Proposição 3.3.2 o germe de campo de vetores $\eta_{t}=\sum_{i=1}^{n}\left(X_{i} \frac{\partial}{\partial x_{i}}+Y_{i} \frac{\partial}{\partial y_{i}}\right)$ é levantável sobre $F$.

De acordo com as Proposições 1.2.18 e 3.3.5 temos que $\mathcal{L}_{\eta_{t}} \sigma_{t}^{(4)}$ é uma 2-forma fechada homogênea de grau 5 que se anula em $T L_{\text {reg. }}$. Segue da Proposição 3.2.6 que $\mathcal{L}_{\eta_{t}} \sigma_{t}^{(4)}$ tem restrição algébrica a $L$ nula.

Determinamos os germes de funções $f_{i j k l}$ pelas EDO's

$$
i_{\eta_{t}}(1-t) \sigma^{(3)}=-\sum_{\substack{1 \leq i \leq j \leq k \leq n, 1 \leq l \leq n}} \frac{d f_{i j k l}(t)}{d t}\left(x_{i} x_{j} y_{k} d x_{l}-x_{i} x_{j} y_{k} d y_{l}\right)
$$

com condição inicial $f_{i j k}(0)=c_{i j k}$.

Provamos assim que

$$
\mathcal{L}_{\eta_{t}}\left(\sigma^{(2)}+(1-t) \sigma_{(3)}\right)=\sigma^{(3)}-\frac{d}{d t} \sigma_{t}^{(4)}
$$

Logo a família de difeomorfismos $\Phi_{t}$ associada a $\eta_{t}$ preserva $L$, pois $\eta_{t}$ é levantável sobre $F$ e $\Phi^{*}\left[\sigma_{t}\right]_{L}=[\sigma]_{L}, t \in[0,1]$. Portanto $[\sigma]_{L}$ é difeomorfa a $\left[\sigma^{(2)}+\theta\right]_{L}$, onde

$$
\theta=\sum_{\substack{1 \leq i \leq j \leq k \leq n \\ 1 \leq l \leq n}} \tilde{c}_{i j k}\left(x_{i} x_{j} y_{k} d x_{l}-x_{i} x_{j} y_{k} d y_{l}\right)
$$

onde $\tilde{c}_{i j k}=\frac{d f_{i j k l}}{d t}(1)$

Lema 3.3.8. A restrição algébrica $\left[\sigma^{(2)}+\theta\right]_{L}$ é difeomorfa $a\left[\sigma^{(2)}\right]_{L}$. 
Demonstração. A prova deste Lema é bem similar a do Lema 3.3.7. Considere $\sigma_{t}=\sigma^{(2)}+(1-t) \theta$. Procuramos $\eta_{t}$ um germe de campo de vetores levantável sobre $F$ homogêneo de grau 2 tal que

$$
\mathcal{L}_{\eta_{t}}\left[\sigma_{t}\right]_{L}=\left[\sigma^{(2)}+\theta\right]_{L}
$$

Sendo $\eta_{t}$ homogêneo de grau 2 temos que

$$
\mathcal{L}_{\eta_{t}}\left[\sigma_{t}\right]_{L}=\mathcal{L}_{\eta_{t}}\left[\sigma^{(2)}\right]_{L}
$$

Isso acontece devido às Proposições 3.2.6 e 3.3.5. Se encontrarmos um germe de campo de vetores levantável sobre $F \eta_{t}$ que satisfaz

$$
i_{\eta_{t}} \sigma^{(2)}=\sum_{\substack{1 \leq i \leq j \leq k \leq n, 1 \leq l \leq n}} \tilde{c}_{i j k l}\left(x_{i} x_{j} y_{k} d x_{l}-y_{i} y_{j} x_{k} d y_{l}\right)
$$

então $\eta_{t}$ satisfaz (3.4).

Analogamente ao Lema 3.3.7, para $l=1, \ldots, n$ temos as seguintes igualdades

$$
\left\{\begin{array}{l}
\sum_{s=1}^{n} e_{s l} Y_{s}=-\sum_{1 \leq i \leq j \leq k \leq n} \tilde{c}_{i j k l} x_{i} x_{j} y_{k}, \\
\sum_{s=1}^{n} e_{s l} X_{s}=-\sum_{1 \leq i \leq j \leq k \leq n} \tilde{c}_{i j k l} y_{i} y_{j} x_{k},
\end{array}\right.
$$

onde $\left\{\begin{array}{l}e_{i j}=a_{i j}, i<j \\ e_{i j}=a_{j i}, i>j \\ e_{i i}=2 a_{i i}, i=l, \ldots, n\end{array}\right.$.

Seja $W=\left(w_{i j}\right) \in \mathrm{GL}(n, \mathbb{R})$ a matriz inversa de $E=\left(e_{i j}\right)$. Uma solução para o sistema é 


$$
\begin{aligned}
& X_{1}=-\sum_{1 \leq i \leq j \leq k \leq n}\left(w_{11}\left(\tilde{c}_{i j k 1} x_{i} x_{j} y_{k}\right)+\cdots+w_{1 n}\left(\tilde{c}_{i j k n} x_{i} x_{j} y_{k}\right)\right) \\
& \vdots \\
& X_{n}=-\sum_{1 \leq i \leq j \leq k \leq n}\left(w_{n 1}\left(\tilde{c}_{i j k n} x_{i} x_{j} y_{k}\right)+\cdots+w_{n n}\left(\tilde{c}_{i j k n} x_{i} x_{j} y_{k}\right)\right) \\
& Y_{1}=-\sum_{1 \leq i \leq j \leq k \leq n}\left(w_{11}\left(\tilde{c}_{i j k 1} y_{i} y_{j} x_{k}\right)+\cdots+w_{1 n}\left(\tilde{c}_{i j k n} y_{i} y_{j} x_{k}\right)\right) \\
& \vdots \\
& Y_{n}=-\sum_{1 \leq i \leq j \leq k \leq n}\left(w_{n 1}\left(\tilde{c}_{i j k 1} y_{i} y_{j} x_{k}\right)+\cdots+w_{n n}\left(\tilde{c}_{i j k n} y_{i} y_{j} x_{k}\right)\right)
\end{aligned}
$$

O germe $\eta_{t}$ é levantável sobre $F$ devido à Proposição 3.3.2. Portanto a família de difeomorfismos $\Phi_{t}$ associada a $\eta_{t}$ preserva $L$, pois $\eta_{t}$ é levantável sobre $F$ e $\Phi^{*}\left[\sigma_{t}\right]_{L}=[\sigma]_{L}, t \in[0,1]$. Portanto $\left[\sigma^{(2)}+\theta\right]_{L}$ é difeomorfa a $\left[\sigma^{(2)}\right]_{L}$.

Proposição 3.3.9. Seja $\Phi:\left(\mathbb{R}^{2 n}, 0\right) \rightarrow\left(\mathbb{R}^{2 n}, 0\right)$ uma simetria local de L. Então o germe de difeomorfismo $\Phi^{(1)}$ é uma simetria local de $L$.

Demonstração. Seja $\left\{j_{1}, j_{2}, j_{3}\right\}=\{1,2,3\}$ tal que $\Phi\left(L_{i}\right)=L_{j_{i}}$, para $i=1,2,3$. Seja $p \in L_{i}$, para algum $i \in\{1,2,3\}$. Temos que $\Phi$ se escreve como

$$
\Phi=\Phi^{(1)}+\tilde{\Phi}
$$

onde $\tilde{\Phi}^{(1)}=0$. Pela fórmula da expansão de Taylor temos que

$$
\lim _{t \rightarrow 0} \frac{\Phi(t p)}{t}=\Phi^{(1)}(p)
$$

Como $L_{i}$ é um germe de subespaço linear de $\mathbb{R}^{2 n}$ segue que $\Phi(t p) / t$ pertence ao subespaço linear $\ell_{j_{i}}$ que contém o germe $L_{j_{i}}$, para todo $t \in(0,1]$. Portanto

$$
\lim _{t \rightarrow 0} \frac{\Phi(t p)}{t}=\Phi^{(1)}(p) \in \ell_{j_{i}}
$$

Tomando $p$ suficientemente próximo da origem temos que $\Phi^{(1)}(p) \in L_{j_{i}}$.

Como consequência das Proposições 3.3.6 e 3.3.9, a classificação das restrições algébricas a $L$ de formas simpléticas que se anulam em $T L_{\text {reg }}$ sob a ação das simetrias locais de $L$ se reduz à classificação das restrições algébricas a $L$ de formas simpléticas homogêneas de grau 2 que se anulam em $T L_{\text {reg }}$ sob a ação das simetrias locais de $L$ lineares. Como não existem 2-formas 
homogêneas de grau 2 com restrição algébrica a $L$ nula temos o seguinte resultado.

Proposição 3.3.10. Sejam $\sigma_{1}, \sigma_{2}$ duas formas simpléticas que se anulam em $T L_{\mathrm{reg}}$. Então $\left[\sigma_{1}\right]_{L}$ é difeomorfa a $\left[\sigma_{2}\right]_{L}$ se, e somente se, existe uma simetria local de $L$ linear $\Psi:\left(\mathbb{R}^{2 n}, 0\right) \rightarrow\left(\mathbb{R}^{2 n}, 0\right)$ tal que $\Psi^{*} \sigma_{2}^{(2)}=\sigma_{1}^{(2)}$.

\subsection{Classificação de estrelas lagrangianas em $\left(\mathbb{R}^{2 n}, 0\right)$}

Seja $\Phi:\left(\mathbb{R}^{2 n}, 0\right) \rightarrow\left(\mathbb{R}^{2 n}, 0\right)$ uma simetria local de $L$ linear com $\Phi\left(L_{i}\right)=L_{i}$, para $i \in\{1,2,3\}$. Considere as matrizes $B, C, E, F \in M(n, \mathbb{R})$ tais que $\Phi$ tem a matriz associada

$$
\left[\begin{array}{ll}
B & C \\
E & F
\end{array}\right]
$$

Seja $(x, 0)=\left(x_{1}, \ldots, x_{n}, 0, \ldots, 0\right) \in L_{1}$ então

$$
\left[\begin{array}{ll}
B & C \\
E & F
\end{array}\right]\left[\begin{array}{l}
x \\
0
\end{array}\right]=\left[\begin{array}{c}
B x \\
E x
\end{array}\right] .
$$

Segue que $E=0$. Analogamente, como $\Phi\left(L_{2}\right)=L_{2}$ então $C=0$. Como $\Phi\left(L_{3}\right)=L_{3}$ então $F=B$. Portanto $\Phi:\left(\mathbb{R}^{2 n}, 0\right) \rightarrow\left(\mathbb{R}^{2 n}, 0\right)$ tem matriz associada

$$
\left[\begin{array}{ll}
B & 0 \\
0 & B
\end{array}\right]
$$

Como $\Phi$ é um isomorfismo segue que $B \in \mathrm{GL}(n, \mathbb{R})$.

Para o caso geral, onde $\Psi:\left(\mathbb{R}^{2 n}, 0\right) \rightarrow\left(\mathbb{R}^{2 n}, 0\right)$ é uma simetria local de $L$ linear satisfazendo $\Psi\left(L_{i}\right)=L_{j_{i}}$, onde $\left\{j_{1}, j_{2}, j_{3}\right\}=\{1,2,3\}$ repetimos o mesmo processo para obter o seguinte resultado.

Proposição 3.4.1. Seja $\Phi:\left(\mathbb{R}^{2 n}, 0\right) \rightarrow\left(\mathbb{R}^{2 n}, 0\right)$ uma simetria local de L linear. Então $\Phi$ tem uma das representações matriciais a seguir:
1. $\left[\begin{array}{cc}B & 0 \\ 0 & B\end{array}\right]$
3. $\left[\begin{array}{cc}B & 0 \\ B & -B\end{array}\right]$
5. $\left[\begin{array}{cc}0 & B \\ -B & B\end{array}\right]$
2. $\left[\begin{array}{cc}0 & B \\ B & 0\end{array}\right]$
4. $\left[\begin{array}{cc}B & -B \\ 0 & -B\end{array}\right]$
6. $\left[\begin{array}{cc}B & -B \\ B & 0\end{array}\right]$ 
onde $B \in \mathrm{GL}(n, \mathbb{R})$.

Seja $\sigma$ uma forma simplética homogênea de grau 2 que se anula em $T L_{\text {reg. }}$. De acordo com a Proposição 3.2.6, $\sigma$ se escreve como $\sigma=\sum_{i, j=1}^{n} a_{i j} d x_{i} \wedge d y_{j}$, onde $a_{i j}=a_{j i}, i, j=1, \ldots, n$. Como $\sigma$ é simplética segue que $\operatorname{det} A \neq 0$, onde $A=\left(a_{i j}\right)$. Para todo $p \in\left(\mathbb{R}^{2 n}, 0\right)$, a forma bilinear $\sigma(p): \mathbb{R}^{2 n} \times \mathbb{R}^{2 n} \rightarrow \mathbb{R}$ tem matriz associada

$$
W=\left[\begin{array}{cc}
0 & A \\
-A & 0
\end{array}\right]
$$

Seja $\Phi:\left(\mathbb{R}^{2 n}, 0\right) \rightarrow\left(\mathbb{R}^{2 n}, 0\right)$ uma simetria local de $L$ linear com representação matricial do tipo (1) da Proposição 3.4.1. O pullback de $\Phi$ em $\sigma$ é dado por

$$
\text { (1) }\left[\begin{array}{ll}
B & 0 \\
0 & B
\end{array}\right]^{T}\left[\begin{array}{cc}
0 & A \\
-A & 0
\end{array}\right]\left[\begin{array}{ll}
B & 0 \\
0 & B
\end{array}\right]=\left[\begin{array}{cc}
0 & B^{T} A B \\
-B^{T} A B & 0
\end{array}\right] \text {. }
$$

Para as demais simetrias locais de $L$ lineares $\Phi$ descritas na Proposição 3.4.1, $\Phi^{*} \sigma$ tem uma das representações matriciais
(2) $\left[\begin{array}{cc}0 & -B^{T} A B \\ B^{T} A B & 0\end{array}\right]$
(3) $\left[\begin{array}{cc}0 & -B^{T} A B \\ B^{T} A B & 0\end{array}\right]$
(4) $\left[\begin{array}{cc}0 & -B^{T} A B \\ B^{T} A B & 0\end{array}\right]$
(5) $\left[\begin{array}{cc}0 & B^{T} A B \\ -B^{T} A B & 0\end{array}\right]$
(6) $\left[\begin{array}{cc}0 & B^{T} A B \\ -B^{T} A B & 0\end{array}\right]$.

Definição 3.4.2. Duas matrizes $A, B \in M(n, \mathbb{R})$ são congruentes se existe uma matriz $P \in \operatorname{GL}(n, \mathbb{R})$ tal que

$$
A=P^{T} B P .
$$

Portanto classificar restrições algébricas a $L$ de formas simpléticas homogêneas de grau 2 
que se anulam em $T L_{\text {reg }}$ sob a ação das simetrias locais de $L$ lineares é equivalente a classificar matrizes simétricas em $\operatorname{GL}(n, \mathbb{R})$ sob a relação de congruência. Para tal utilizamos a Lei de Inércia de Sylvester.

Para tripla de inteiros não negativos $(u, m, k)$ tais que $n=u+m+k$ definimos a matriz em $M(n, \mathbb{R})$

$$
Z_{u, m, k}=\left[\begin{array}{ccc}
I d_{u} & 0 & 0 \\
0 & -I d_{m} & 0 \\
0 & 0 & 0
\end{array}\right]
$$

Teorema 3.4.3 (Lei de Inércia de Sylvester [R]). Seja $S_{n}$ o conjunto das matrizes simétricas $n \times n$ com coeficientes reais. Então:

1. Qualquer matriz em $S_{n}$ é congruente a uma única matriz da forma $Z_{u, m, k}$ para inteiros não negativos $u, m, k$ tais que $n=u+m+k$.

2. Cada classe de equivalência de $S_{n}$, segundo a relação de congruência, tem um representante da forma $Z_{u, m, k}$.

3. Seja $M \in S_{n}$ congruente a $Z_{u, m, k}$. Então $u+m$ é o posto de $M$ e $u-m$ é a assinatura de $M$. O par $(u, m)$, ou equivalentemente o par $(u+m, u-m)$, é um sistema completo de invariantes para a congruência em $S_{n}$.

Para cada $s \in\{0, \ldots, n\}$, definamos $a_{s}=\left[\omega_{s}\right]_{L}$ onde

$$
\omega_{s}=d x_{1} \wedge d y_{1}+\cdots+d x_{s} \wedge d y_{s}-d x_{s+1} \wedge d y_{s+1}-\cdots-d x_{n} \wedge d y_{n}
$$

Teorema 3.4.4. Seja $\sigma$ uma forma simplética em $\left(\mathbb{R}^{2 n}, 0\right)$ que se anula em $T L_{\mathrm{reg}}$. Então $[\sigma]_{L}$ é difeomorfa a uma, e somente uma, restrição algébrica a $L$ do tipo $a_{s}$, para algum $s \in\{0, \ldots, n\}$ com $s \leq \frac{n}{2}$.

Demonstração. De acordo com a Proposição 3.3.10, é suficiente mostrar que dada $\sigma$ uma forma simplética homogênea de grau 2 que se anula em $T L_{\text {reg }}$ existe $\Psi:\left(\mathbb{R}^{2 n}, 0\right) \rightarrow\left(\mathbb{R}^{2 n}, 0\right)$ uma simetria local de $L$ linear tal que $\Psi^{*} \sigma=\omega_{s}$, para um único $s \in\{0, \ldots, n\}$ tal que $s \leq \frac{n}{2}$. De acordo com a Proposição 3.2.6, para todo $p \in\left(\mathbb{R}^{2 n}, 0\right)$ a forma bilinear $\sigma(p): \mathbb{R}^{2 n} \times \mathbb{R}^{2 n} \rightarrow \mathbb{R}$ tem a matriz associada

$$
W=\left[\begin{array}{cc}
0 & A \\
-A & 0
\end{array}\right]
$$

onde $A \in \mathrm{GL}(n, \mathbb{R})$ é simétrica.

De acordo com o Teorema 3.4.3, existe $B \in \mathrm{GL}(n, \mathbb{R})$ tal que $B^{T} A B$ é igual a uma, e somente uma, das matrizes $Z_{u, n-u, 0}$ para algum $u \in\{0, \ldots, n\}$ onde 


$$
Z_{u, n-u, 0}=\left[\begin{array}{cc}
I d_{u} & 0 \\
0 & -I d_{n-u}
\end{array}\right]
$$

Se $u \leq \frac{n}{2}$ considere $\Phi$ a simetria local de $L$ linear com representação matricial

$$
\left[\begin{array}{ll}
B & 0 \\
0 & B
\end{array}\right]
$$

A forma bilinear $\Phi^{*} \sigma(p)$ tem representação matricial

$$
\left[\begin{array}{cc}
0 & Z_{u, n-u, 0} \\
-Z_{u, n-u, 0} & 0
\end{array}\right] .
$$

Logo, $\Phi^{*} \sigma=\sum_{i=1}^{u} d x_{i} \wedge d y_{i}-\sum_{i=u+1}^{n} d x_{i} \wedge d y_{i}=\omega_{u}$

Se $u>\frac{n}{2}$ considere $\Psi$ a simetria local de $L$ linear com matriz associada

$$
\left[\begin{array}{ll}
0 & B \\
B & 0
\end{array}\right]
$$

Portanto $\Psi^{*} \sigma(p)$ tem representação matricial

$$
\left[\begin{array}{cc}
0 & -Z_{u, n-u, 0} \\
Z_{u, n-u, 0} & 0
\end{array}\right]
$$

De acordo com o Teorema 3.4.3, existe $C \in \operatorname{GL}(n, \mathbb{R})$ tal que $C^{T}\left(-Z_{u, n-u, 0}\right) C=Z_{n-u, u, 0}$. Seja $H$ a simetria local de $L$ linear com matriz associada

$$
\left[\begin{array}{ll}
C & 0 \\
0 & C
\end{array}\right]
$$

Temos que

$$
H^{*} \Psi^{*} \sigma=\sum_{i=1}^{n-u} d x_{i} \wedge d y_{i}-\sum_{i=n-u+1}^{n} d x_{i} \wedge d y_{i}=\omega_{n-u}
$$

Segue que as órbitas das restrições algébricas a $L$ de formas simpléticas sob a ação do grupo das simetrias locais de $L$ tem um representante $\omega_{s}$, para $s \leq \frac{n}{2}$. 
Agora resta provar que as órbitas de $a_{1}, \ldots, a_{s}$ são disjuntas, onde $s$ é o maior inteiro tal que $s \leq \frac{n}{2}$. Para isso, sejam $u, v \in\{0, \ldots, n\}, u, v \leq \frac{n}{2}$ e $u \neq v$. As representações matriciais de $\omega_{u}(p)$ e $\omega_{v}(p)$ são respectivamente:

$$
\left[\begin{array}{cc}
0 & Z_{u, n-u, 0} \\
-Z_{u, n-u, 0} & 0
\end{array}\right] \text { e }\left[\begin{array}{cc}
0 & Z_{v, n-v, 0} \\
-Z_{v, n-v, 0} & 0
\end{array}\right] \text {. }
$$

Observe que as assinaturas de $Z_{v, n-v, 0} \mathrm{e}-Z_{v, n-v, 0}$ são diferentes de $Z_{u, n-u, 0}$. De acordo com o Teorema 3.4.3, a matriz $Z_{u, n-u, 0}$ não é congruente nem a $Z_{v, n-v, 0}$ nem a $-Z_{v, n-v, 0}$. Segue da Proposição 3.4.1 que não existe uma simetria local de $L$ linear $T:\left(\mathbb{R}^{2 n}, 0\right) \rightarrow\left(\mathbb{R}^{2 n}, 0\right)$ tal que $T^{*} a_{u}=a_{v}$. Portanto as órbitas representadas por $a_{u}$ e $a_{v}$ são disjuntas.

Teorema 3.4.5. Uma estrela lagrangiana transversal é equivalente a uma, e somente uma, das seguintes estrelas lagrangianas $E^{s}=\left(\left\{L_{1}, L_{2}, L_{3}^{s}\right\}, 0\right)$, onde $L_{1}=\left\{y_{1}=\cdots=y_{n}=0\right\}, L_{2}=\left\{x_{1}=\cdots=\right.$ $\left.x_{n}=0\right\}$ e $L_{3}^{s}=\left\{x_{1}-y_{1}=\cdots=x_{s}-y_{s}=x_{s+1}+y_{s+1}=\cdots=x_{n}+y_{n}=0\right\}$.

Demonstração. De acordo com o Teorema 3.4.4, as órbitas das restrições algébricas a $L$ de formas simpléticas que se anulam em $T L_{\text {reg }}$ são

$$
a_{1}=\left[\omega_{1}\right]_{L}, \ldots, a_{u}=\left[\omega_{u}\right]_{L},
$$

onde $u$ é o maior inteiro que satisfaz $u \leq \frac{n}{2}$.

Seja $s$ um inteiro positivo tal que $s \leq u$. Considere o germe de difeomorfismo $\Phi_{s}:\left(\mathbb{R}^{2 n}, 0\right) \rightarrow$ $\left(\mathbb{R}^{2 n}, 0\right)$ definido por $\Phi_{s}(x, y)=\left(x, y_{1}, \ldots, y_{s},-y_{s+1}, \ldots,-y_{n}\right)$. Observe que $\Phi_{s}^{*} \omega_{s}=\omega$. Logo as restrições algébricas $a_{1}, \ldots, a_{u}$ são difeomorfas a

$$
[\omega]_{\Phi_{1}^{-1}(L)}, \ldots,[\omega]_{\Phi_{u}^{-1}(L)},
$$

respectivamente. Sejam $E^{s}=\left(\left\{\Phi_{s}^{-1}\left(L_{1}\right), \Phi_{s}^{-1}\left(L_{2}\right), \Phi_{s}^{-1}\left(L_{3}\right)\right\}, 0\right)$. De acordo com o Lema 3.1.2 segue que cada $E^{s}$ é uma estrela lagrangiana em $\left(\left(\mathbb{R}^{2 n}, \omega\right), 0\right)$. Segue do Corolário 3.1.3 que as estrelas lagrangianas

$$
E^{1}, \ldots, E^{u}
$$

formam uma lista de estrelas lagrangianas transversais sob a ação dos simplectomorfismos.

\section{5 Índice de Maslov}

Um invariante natural para a classificação simplética da união de 3 subespaços lineares lagrangianos é o índice de Maslov [LV]. Nesta seção descrevemos o índice de maslov para 
estrelas lagrangianas transversais e mostramos que ele é um invariante simplético. Para mais detalhes sobre o índice de Maslov veja [LV].

Sejam $V_{1}, V_{2}, V_{3}$ subespaços lagrangianas do espaço vetorial simplético $\left(\mathbb{R}^{2 n}, B\right)$, onde $B$ é uma forma bilinear simplética.

Definição 3.5.1. O indice de Maslov de $V_{1}, V_{2}, V_{3}$, denotado por $\tau_{B}\left(V_{1}, V_{2}, V_{3}\right)$, é a assinatura da forma quadrática $Q_{B}: V_{1} \oplus V_{2} \oplus V_{3} \rightarrow \mathbb{R}$ definida por

$$
Q_{B}\left(v_{1}, v_{2}, v_{3}\right)=B\left(v_{1}, v_{2}\right)+B\left(v_{2}, v_{3}\right)+B\left(v_{3}, v_{1}\right), v_{i} \in V_{i}, i=1,2,3 .
$$

$A$ assinatura de $Q_{B}$ é definida da seguinte forma: existe um base de $V_{1} \oplus V_{2} \oplus V_{3}$, onde a matriz de $Q_{B}$ é diagonal e contém $p$ vezes o número $1, q$ vezes o número -1 e $r$ vezes o número 0 . Assinatura de $Q_{B}$ é igual a $p-q$.

Proposição 3.5.2. ([LV])

1. Para todo $\Phi$ simplectomorfismo linear de $\left(\mathbb{R}^{2 n}, B\right)$ tem-se: $\tau_{B}\left(\Phi\left(V_{1}\right), \Phi\left(V_{2}\right), \Phi\left(V_{3}\right)\right)=\tau_{B}\left(V_{1}, V_{2}, V_{3}\right)$.

2. $\tau_{B}\left(V_{1}, V_{2}, V_{3}\right)=-\tau_{B}\left(V_{2}, V_{1}, V_{3}\right)=-\tau_{B}\left(V_{1}, V_{3}, V_{2}\right)$.

Proposição 3.5.3. ([LV]) Sejam $V_{1}, V_{2}$ e $V_{3}$ subespaços lagrangianos dois a dois transversais em $\left(\mathbb{R}^{2 n}, B\right)$. Então existe uma base simplética $p_{1}, \ldots, p_{n}, q_{1}, \ldots, q_{n}$ e um inteiro $s, 0 \leq s \leq n$, tais que

$$
\begin{aligned}
& V_{1}=\left\{q_{1}=\cdots=q_{n}=0\right\} \\
& V_{2}=\left\{p_{1}=\cdots=p_{n}=0\right\} \\
& V_{3}^{s}=\left\{p_{1}-q_{1}=\cdots=p_{s}-q_{s}=p_{s+1}+q_{s+1}=\cdots=p_{n}+q_{n}=0\right\} .
\end{aligned}
$$

Portanto $\tau_{B}\left(V_{1}, V_{2}, V_{3}\right)=n-2 s$.

Observe que a descrição de estrela lagrangiana é feita sem distinção da ordem das subvariedades lagrangianas apresentadas. Segue da Proposição 3.5.2 que uma generalização do índice de Maslov para estrelas lagrangianas deve ser feito em valor absoluto.

Definição 3.5.4. Seja $E=\left(\left\{L_{1}, L_{2}, L_{3}\right\}, 0\right)$ uma estrela lagrangiana em $\left(\left(\mathbb{R}^{2 n}, \omega\right), 0\right)$. Definimos o indice de Maslov de E como o valor absoluto de $\tau_{\omega}\left(T_{0} L_{1}, T_{0} L_{2}, T_{0} L_{3}\right)$. Denotamos o indice de Maslov de $E \operatorname{por} \tau_{\omega}(E)$.

Proposição 3.5.5. Sejam $E=\left(\left\{L_{1}, L_{2}, L_{3}\right\}\right)$ e $E^{\prime}=\left(\left\{L_{1}{ }^{\prime}, L_{2}{ }^{\prime}, L_{3}{ }^{\prime}\right\}\right)$ duas estrelas lagrangianas transversais em $\left(\left(\mathbb{R}^{2 n}, \omega\right), 0\right)$ equivalentes. Então $\tau_{\omega}(E)=\tau_{\omega}\left(E^{\prime}\right)$. 
Demonstração. Sendo $E$ e $E^{\prime}$ equivalentes, então existe um germe de simplectomorfismo $\Phi$ : $\left(\left(\mathbb{R}^{2 n}, \omega\right), 0\right) \rightarrow\left(\left(\mathbb{R}^{2 n}, \omega\right), 0\right)$ tal que $\Phi\left(L_{i}\right)=L_{j_{i}}{ }^{\prime}$, para uma permutação $j_{1}, j_{2}, j_{3}$ de $1,2,3$. Portanto

$$
d \Phi(0)\left(T_{0} L_{i}\right)=T_{0} L_{j_{i}}{ }^{\prime}, \quad i=1,2,3
$$

Note que $d \Phi(0)$ é um simplectomorfismo linear do espaço vetorial simplético $\left(\mathbb{R}^{2 n}, \omega(0)\right)$. De acordo com a Proposição 3.5.3 existem simplectomorfismos lineares $\Psi_{1}:\left(\mathbb{R}^{2 n}, \omega(0)\right) \rightarrow$ $\left(\mathbb{R}^{2 n}, \omega(0)\right)$ e $\Psi_{2}:\left(\mathbb{R}^{2 n}, \omega(0)\right) \rightarrow\left(\mathbb{R}^{2 n}, \omega(0)\right)$ tais que

$$
\begin{gathered}
\Psi_{1}\left(T_{0} L_{1}\right) \cup \Psi_{1}\left(T_{0} L_{2}\right) \cup \Psi_{1}\left(T_{0} L_{3}\right)=V_{1} \cup V_{2} \cup V_{3}^{s} \\
\Psi_{2}\left(T_{0} L_{1}{ }^{\prime}\right) \cup \Psi_{2}\left(T_{0} L_{2}{ }^{\prime}\right) \cup \Psi_{2}\left(T_{0} L_{3}{ }^{\prime}\right)=V_{1} \cup V_{2} \cup V_{3}^{s}
\end{gathered}
$$

para algum $s \in\{0, \ldots, n\}$ onde $V_{1}, V_{2}$ e $V_{3}^{s}$ são como na Proposição 3.5.3. De acordo com a Proposição 3.5.2, segue que $\left|\tau_{\omega}\left(T_{0} L_{1}, T_{0} L_{2}, T_{0} L_{3}\right)\right|=\left|\tau_{\omega}\left(T_{0} L_{1}{ }^{\prime}, T_{0} L_{2}{ }^{\prime}, T_{0} L_{3}{ }^{\prime}\right)\right|=\left|\tau_{\omega}\left(V_{1}, V_{2}, V_{3}^{s}\right)\right|$. 


\section{Capítulo \\ 4 \\ Alguns algoritmos desenvolvidos no Singular}

Neste capítulo apresentamos alguns algoritmos desenvolvidos no software Singular [DGPS]. Primeiramente, encontraremos os geradores do ideal $I(g)$ dos polinômios em $\mathbb{R}\left[x_{1}, \ldots, x_{m}\right]$ que se anulam na imagem da curva $g: \mathbb{R} \rightarrow \mathbb{R}^{m}$ dada por $g(t)=\left(t^{\lambda_{1}}, \ldots, t^{\lambda_{m}}\right)$, onde $\lambda_{1}, \ldots, \lambda_{m}$ são inteiros não negativos.

Definição 4.0.6. Um anel A é noetheriano se todo ideal é finitamente gerado.

Teorema 4.0.7. (Teorema da base de Hilbert)([AM2]) Se A é um anel noetheriano então A[x] também é noetheriano.

De acordo com o Teorema 4.0.7, o anel $\mathbb{R}\left[x_{1}, \ldots, x_{m}\right]$ é noetheriano. Seja $I_{Z}(g)=I(g) \cap$ $\mathbb{Z}\left[x_{1}, \ldots, x_{m}\right]$.

Proposição 4.0.8. O ideal $I(g)$ é gerado por polinômios em $I_{Z}(g)$.

Demonstração. Observe que se $P \in I(g)$ então a parte quase homogênea de $P$ de quase grau $l$ pertence a $I(g)$, para todo inteiro não negativo $l$. Aqui consideramos as variáveis pesadas $x_{1}, \ldots, x_{m}$ com pesos $\lambda_{1}, \ldots, \lambda_{m}$, respectivamente. Portanto é suficiente provar que os polinômios quase homogêneos em $I(g)$ são gerados por polinômios em $I_{Z}(g)$.

Seja $h$ um polinômio quase homogêneo em $I(g)$ de quase grau $s$. Temos que $h$ se escreve como

$$
h(x)=\sum a_{i_{1} \ldots i_{m}} x_{1}^{i_{1}} \cdots x_{m}^{i_{m}}
$$

onde $i_{1}, \ldots, i_{m}$ são inteiros não negativos tais que $x_{1}^{i_{1}} \cdots x_{m}^{i_{m}}$ é um monômio de quase grau $s$. Observe que se $x_{1}^{i_{1}} \cdots x_{m}^{i_{m}}$ e $x_{1}^{j_{1}} \cdots x_{m}^{j_{m}}$ são monômios de quase grau $s$ então $x_{1}^{i_{1}} \cdots x_{m}^{i_{m}}-$ $x_{1}^{j_{1}} \cdots x_{m}^{j_{m}} \in I_{Z}(g)$. Fixamos $u_{1}, \ldots, u_{m}$ inteiros não negativos tais que $x_{1}^{u_{1}} \cdots x_{m}^{u_{m}}$ é quase homogêneo de quase grau $s$. Então $h$ se escreve como 


$$
h(x)=\left(\sum a_{i_{1} \ldots i_{m}}\right) x_{1}^{u_{1}} \cdots x_{m}^{u_{m}}+\sum_{\left(i_{1}, \ldots, i_{m}\right) \neq\left(u_{1}, \ldots, u_{m}\right)} a_{i_{1} \ldots i_{m}}\left(x_{1}^{i_{1}} \ldots x_{m}^{i_{m}}-x_{1}^{u_{1}} \cdots x_{m}^{u_{m}}\right)
$$

Restringindo $h$ a $\operatorname{Im}(g)$ concluímos que $\sum a_{i_{1} \ldots i_{m}}=0$. Portanto $h$ é gerado por polinômios em $I_{Z}(g)$.

Utilizamos o software Singular para encontrar geradores de $I(g)$. Como exemplo, vejamos o ideal de polinômios em $\mathbb{R}\left[x_{1}, x_{2}, x_{3}, x_{4}\right]$ que se anulam na curva $t \rightarrow\left(t^{4}, t^{5}, t^{6}, t^{7}\right)$.

Exemplo 4.0.9. Geradores do ideal dos polinômios que se anulam na curva $t \rightarrow\left(t^{4}, t^{5}, t^{6}, t^{7}\right)$ em $\mathbb{R}\left[x_{1}, x_{2}, x_{3}, x_{4}\right]$.

>LIB "all.lib";

>ring $r=0,(x 1, x 2, x 3, x 4, t), d p ; / / a n e l$ dos polinômios com ordem

lexicográfica reversa.

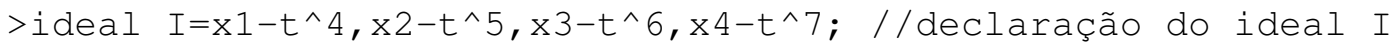

>eliminate(II,t); //geradores do ideal dos polinômios que se anulam na curva $\$$ t $\backslash$ to $\left(t^{\wedge} 4, t^{\wedge} 5, t^{\wedge} 6, t^{\wedge} 7\right) \$$.

O processo para encontrar os geradores de $I(g)$, onde $g(t)=\left(t^{4}, t^{5}, t^{6}\right)$ e $\left(t^{4}, t^{5}, t^{7}\right)$, é análogo.

No que segue, apresentamos um algoritmo para encontrar geradores do espaço vetorial das restrições algébricas a $g(t)=\left(t^{4}, t^{5}, t^{6}, t^{7}\right)$ de 2-formas fechadas em $\left(\mathbb{R}^{4}, 0\right)$. Devido ao Teorema 1.1.10, é suficiente encontrar uma base para o espaço vetorial das restrições algébricas a $g$ de 2-formas exatas.

Denotaremos por $\Lambda_{p}^{k}\left(\mathbb{R}^{m}\right)$ o conjunto das $k$-formas em $\mathbb{R}^{m}$ do tipo $\sum f_{i_{1} \ldots i_{k}} d x_{i_{1}} \wedge \ldots d x_{i_{k}}$, onde $f_{i_{1} \ldots i_{k}}$ é um polinômio em $\mathbb{R}^{m}$. Seja $W(m)=\Lambda_{p}^{0}\left(\mathbb{R}^{m}\right)+\Lambda_{p}^{1}\left(\mathbb{R}^{m}\right)+\Lambda_{p}^{2}\left(\mathbb{R}^{m}\right)$. O conjunto $W(m)$ é um anel com as operações de soma e produto exterior $(+, \wedge)$ e pode ser identificado com o anel quociente $\mathbb{R}\left[x_{1}, \ldots, x_{m}, d x_{1}, \ldots, d x_{m}\right] / S$, onde $S$ é o ideal gerado pelas relações $d x_{i} d x_{j}=-d x_{j} d x_{i}$ e $d x_{i} d x_{j} d x_{l}=0, i, j, l \in\{1, \ldots, m\}$. Definimos os seguintes ideais em $W(m)$ :

- $\mathcal{M}$ o ideal gerado por $x_{1}, \ldots, x_{m}$;

- $d \mathcal{M}$ o ideal gerado pelas derivadas exteriores dos geradores de $\mathcal{M}$, isto é, pelas 1-formas $d x_{1}, \ldots, d x_{m}$

- $d \mathcal{M}^{2}$ o ideal gerado pelos produtos exteriores dos geradores de $d \mathcal{M}$, isto é, gerado por elementos da forma: $d x_{i} \wedge d x_{j}, i, j \in\{1, \ldots, m\}$;

- $d I(g)$ o ideal gerado pelas derivadas exteriores dos polinômios geradores do ideal $I(g)$; 
- $I(g) \wedge d \mathcal{M}^{2}$ o ideal gerado pelos produtos exteriores dos geradores de $I(g)$ com geradores de $d \mathcal{M}^{2}$;

- $d I(g) \wedge d \mathcal{M}$ o ideal gerado pelos produtos exteriores dos geradores de $d I(g)$ com geradores de $d \mathcal{M}$;

- $Z=I(g) \wedge d \mathcal{M}^{2}+d I(g) \wedge d \mathcal{M}$ o ideal gerado pelas somas dos geradores de $I(g) \wedge d \mathcal{M}^{2}$ e $d I(g) \wedge d \mathcal{M}$.

Lema 4.0.10. Seja $\tilde{Z}$ o conjunto das 2 -formas $\alpha+d \beta$, onde $\alpha \in \Lambda_{p}^{2}\left(\mathbb{R}^{m}\right)$ se anula em $\operatorname{Im}(g)$ e $\beta \in$ $\Lambda_{p}^{1}\left(\mathbb{R}^{m}\right)$ se anula em $\operatorname{Im}(g)$. Então $Z=\tilde{Z}$.

Demonstração. De acordo com o Teorema 4.0.7, o ideal $I(g)$ é finitamente gerado. Sejam $g_{1}, \ldots, g_{s}$ os geradores de $I(g)$. Considere $\alpha+d \beta \in \tilde{Z}$. Por definição, $\alpha$ e $\beta$ se escrevem como:

$$
\alpha=\sum_{i, j=1}^{m} f_{i j} d x_{i} \wedge d x_{j} \text {, onde } f_{i j} \in I(g) \text { e } \beta=\sum_{j=1}^{m} h_{j} d x_{j} \text {, onde } h_{j} \in I(g) \text {. }
$$

Sejam $v_{i j 1}, \ldots, v_{i j s}, u_{j 1}, \ldots, u_{j s}, i, j=1, \ldots, s$, polinômios em $\mathbb{R}\left[x_{1}, \ldots, x_{m}\right]$ tais que $f_{i j}=$ $\sum_{l=1}^{s} v_{i j l} g_{l}$ e $h_{j}=\sum_{l=1}^{s} u_{j l} g_{l}$. Temos que

$$
\begin{aligned}
& \alpha+d \beta=\sum_{i, j=1}^{m} f_{i j} d x_{i} \wedge d x_{j}+\sum_{j=1}^{m} d\left(h_{j} d x_{j}\right) \\
& =\sum_{l=1}^{s}\left(\sum_{i, j=1}^{m} v_{i j l} g_{l} d x_{i} \wedge d x_{j}+\sum_{j=1}^{m} d\left(u_{j l} g_{l}\right) \wedge d x_{j}\right) \\
& =\sum_{l=1}^{s}\left(\sum_{i, j=1}^{m} v_{i j l} g_{l} d x_{i} \wedge d x_{j}+\sum_{j=1}^{m} u_{j l} d g_{l} \wedge d x_{j}+\sum_{j=1}^{m} g_{l} d u_{j l} \wedge d x_{j}\right) \\
& =\sum_{l=1}^{s}\left(\sum_{i, j=1}^{m} v_{i j l} g_{l} d x_{i} \wedge d x_{j}+\sum_{j=1}^{m} u_{j l} d g_{l} \wedge d x_{j}+\sum_{i, j=1}^{m} g_{l} \frac{\partial u_{j l}}{\partial x_{i}} d x_{i} \wedge d x_{j}\right) \\
& =\sum_{l=1}^{s}\left(\sum_{i, j=1}^{m}\left(v_{i j l}+\frac{\partial u_{j l}}{\partial x_{i}}\right) g_{l} d x_{i} \wedge d x_{j}+\sum_{j=1}^{m} u_{j l} d g_{l} \wedge d x_{j}\right) .
\end{aligned}
$$

Portanto $\tilde{Z} \subset Z$. A inclusão $Z \subset \tilde{Z}$ é óbvia.

Segue do Lema 4.0.10 que o conjunto das 2-formas com restrição algébrica a $g$ nula é um ideal em $W(m)$. O próximo exemplo exibe uma base para o espaço vetorial $\left[\Lambda^{2, c l}\left(\mathbb{R}^{4}\right)\right]_{g}$, onde $g(t)=\left(t^{4}, t^{5}, t^{6}, t^{7}\right)$.

\section{Exemplo 4.0.11.}

>LIB "all.lib";

>ring $r=0,(x 1, x 2, x 3, x 4, t), d p ; / / a n e l$ dos polinômios com ordem 


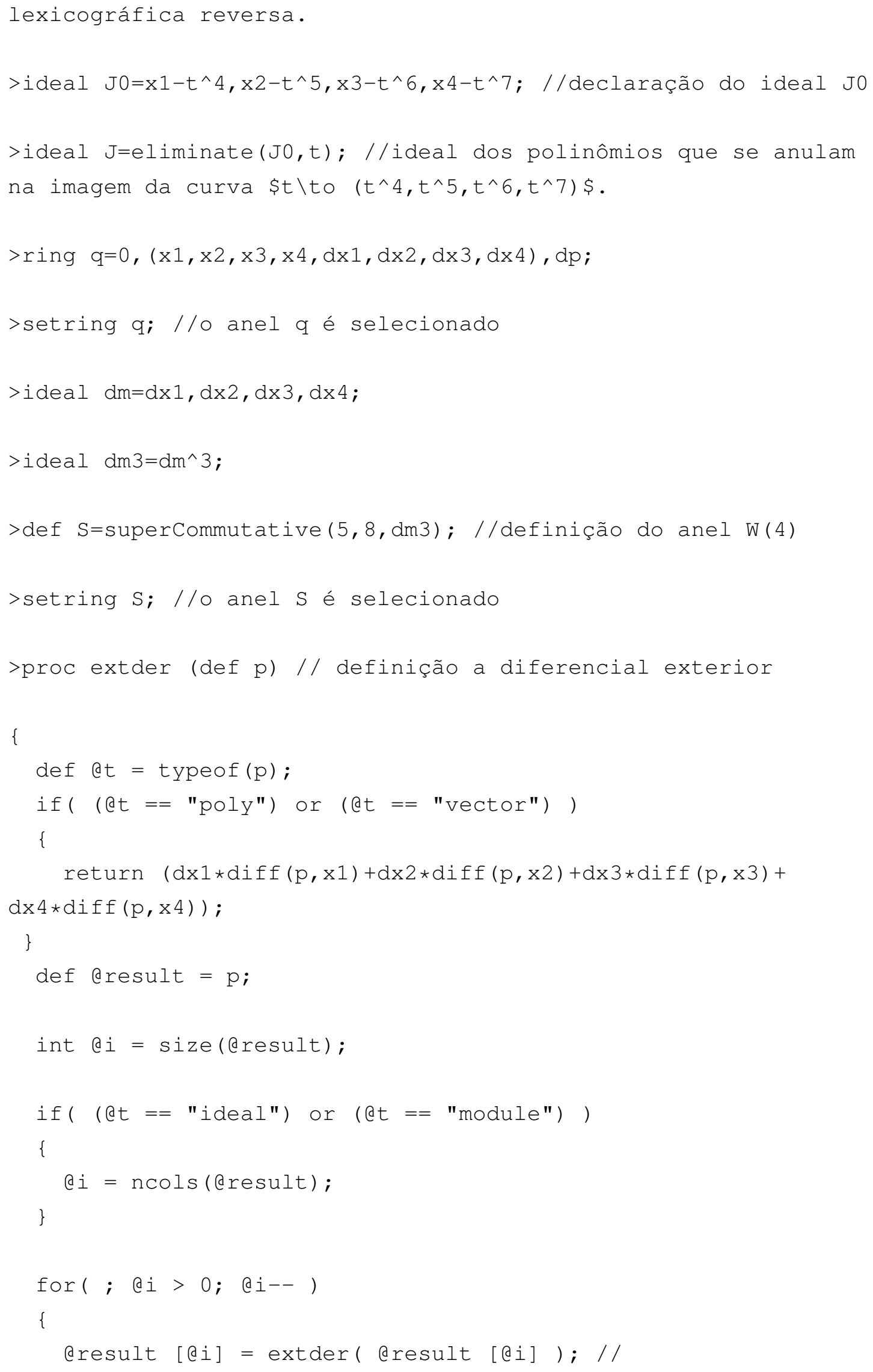




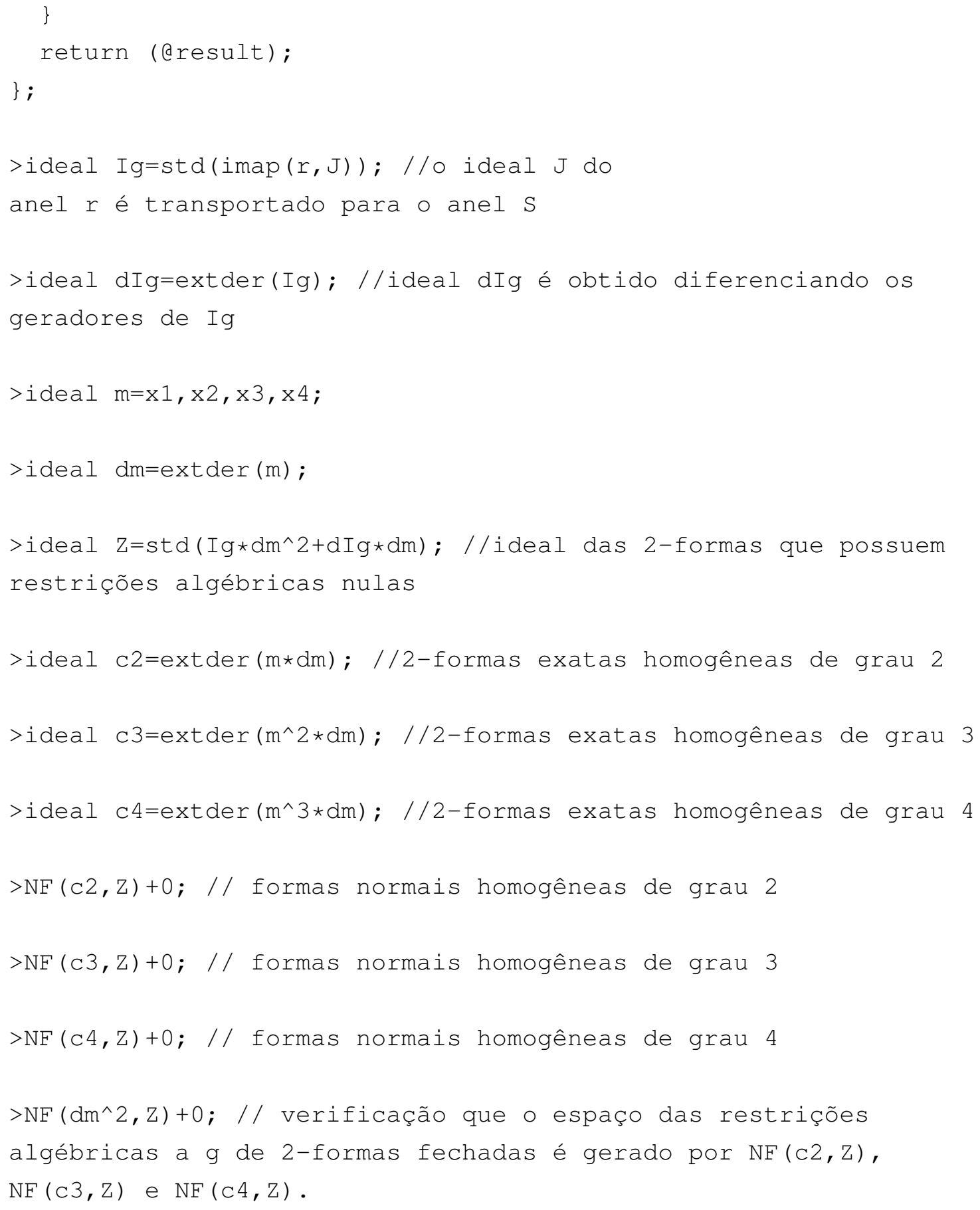

Os algoritmos para determinar bases para os espaços vetoriais das restrições algébricas de 2-formas fechadas a germes de curvas parametrizadas com semigrupos $(4,5,6)$ e $(4,5,7)$ são análogos. Basicamente, a mudança é feita na declaração das variáveis e na declaração dos ideais. 


\section{Referências Bibliográficas}

[A1] Arnold, V. I. First steps of local symplectic algebra. Differential topology, infinitedimensional Lie algebras, and applications, D. B. Fuchs' 60th Anniversary Collection, American Mathematical Society Transl. Ser. 2, Am. Math. Soc. 194(44) 1999, 1-8.

[A2] Arnold, V. I., Simple singularities of curves, Proc. Steklov Inst. Math. no. 3226 (1999), 20-28.

[A3] Cannas da Silva, A., Lectures on symplectic geometry. Lecture Notes in Mathematics, 1764. Sringer-Verlag, Berlin, 2001.

[AG] Arnold, V. I.; Givental, A. B., Symplectic Geometry. Dynamical systems, IV, 1-138, Encyclopedia of Mathematical Sciences, vol. 4, Springer, Berlin, 2001.

[AM1] Abraham, R.; Marsden, J. E., Foundations of mechanics. Second edition, Benjamin/Cummings Publishing Co., Inc., Advanced Book Program, Reading, Mass., 1978.

[AM2] Atiyah, M. F.; Macdonald, I. G., Introduction to commutative algebra. Addison-Wesley Publishing Co., Reading, Mass.-London-Don Mills, Ont. 1969.

[AVG] Arnold, V. I.; Gusein-Zade, S. M.; Varchenko, A. N., Singularities of Differentiable Maps, Vol. 1, The classification of critical points, caustics and wave fronts. Translated from the Russian by Ian Porteous and Mark Reymolds. Monographs in Mathematics, 82 Birkhäuser, Boston, Inc., Boston, MA, 1985.

[BG] Bruce, J. W.; Gaffney, T., Simple singularities of mappings $(\mathbb{C}, 0) \rightarrow\left(\mathbb{C}^{2}, 0\right)$. J. London Math. Soc. 26 (1982) no. 3, 465-474.

[D1] Domitrz, W., Local symplectic algebra of quasi-homogeneous curves. Fund. Math. 204 (2009) no. 1, 57-86.

[D2] Domitrz, W., Zero-dimensional symplectic isolated complete intersection singularities. J. Singul. 6 (2012), 19-26.

[DLW] Domitrz, W.; Lira F.; Wik-Atique R. Symplectic singularity of curves with the semigroup $(4,5,6,7)$, preprint. 
[DGPS] Decker, W.; Greuel, G.-M.; Pfister, G.; Schönemann, H.: Singular 3-1-6 - A computer algebra system for polynomial computations. http://www.singular.uni-kl.de (2013).

[DJZ1] Domitrz, W.; Janeczko, S.; Zhitomirskii, M., Relative Poincaré Lemma, contractibility, quasi-homogeneity and vector fields tangent to a singular variety, Illinois J. Math. 48 (2004), no. 3, 803-835.

[DJZ2] Domitrz, W.; Janeczko S.; Zhitomirskii, M. Symplectic singularities of varieties: the method of algebraic restrictions. J. Reine Angew. Math. 618 (2008), 197-235.

[DT1] Domitrz, W.; Trębska, Ż., Symplectic $S_{\mu}$ singularities, Real and Complex singularities, Contemp. Math. Soc., 569, Amer. Math. Soc., Providence, RI, (2012), 45-65.

[DT2] Domitrz, W.; Trȩbska, Ż., Symplectic $T_{7}, T_{8}$ singularities and Lagrangian tangency orders. Proc. Edinb. Math. Soc. (2) 55 (2012), no. 3, 657-683.

[G1] Gibson, C. G., Singular points of smooth mappings. Research Notes in Mathematics, 25. Pitman (Advanced Publishing Program), Boston, Mass.-London, 1979

[G2] Giusti, M., Classification des singularités isolées d'intersections complètes simples, C. R. Acad. Sci., Paris, Sér. A 284 (1977),167-170

[IJ] Ishikawa, G.; Janeczko, S., Symplectic bifurcations of plane curves and isotropy liftings, Quart. J. Math. 54 (2003), 73-102.

[J] Janeczko, S., Classification of Lagrangian stars and their symplectic reductions, J. Phys. A 31 (1998), no. 16, 3677-3685.

[K] Kolgushkin, P. A., Classification of simple multigerms of curves in a space with symplectic structure. St. Petersburg math. J. 15 (2003), 103-126.

[LV] Lion, G.; Vergne, M., The Weil representation, Maslov index and Theta series. Progress in Mathematics 6, Birkhäuser, Boston, Mass., 1980.

[R] Roman, S., Advanced Linear Algebra. Third edition. Graduate Texts in Mathematics, 135. Springer, New York, (2008).

[S] Stevens, J., Simple curve singularities. Preprint, arXiv:1306.4612.

[T] Trȩbska, Ż., Symplectic $W_{8}$ and $W_{9}$ singularities, J. Singul. 6 (2012), 158-178. 